\title{
INVERSE RANDOM SOURCE PROBLEMS FOR TIME-HARMONIC ACOUSTIC AND ELASTIC WAVES
}

\author{
JIANLIANG LI, TAPIO HELIN, AND PEIJUN LI
}

\begin{abstract}
This paper concerns the random source problems for the time-harmonic acoustic and elastic wave equations in two and three dimensions. The goal is to determine the compactly supported external force from the radiated wave field measured in a domain away from the source region. The source is assumed to be a microlocally isotropic generalized Gaussian random function such that its covariance operator is a classical pseudo-differential operator. Given such a distributional source, the direct problem is shown to have a unique solution by using an integral equation approach and the Sobolev embedding theorem. For the inverse problem, we demonstrate that the amplitude of the scattering field averaged over the frequency band, obtained from a single realization of the random source, determines uniquely the principle symbol of the covariance operator. The analysis employs asymptotic expansions of the Green functions and microlocal analysis of the Fourier integral operators associated with the Helmholtz and Navier equations.
\end{abstract}

\section{INTRODUCTION}

The inverse source scattering in waves, as an important and active research subject in inverse scattering theory, are to determine the unknown sources that generate prescribed radiated wave patterns 32 . It has been considered as a basic mathematical tool for the solution of many medical imaging modalities [2], such as magnetoencephalography (MEG), electroencephalography (EEG), electroneurography (ENG). These imaging modalities are non-invasive neurophysiological techniques that measure the electric or magnetic fields generated by neuronal activity of the brain. The spatial distributions of the measured fields are analyzed to localize the sources of the activity within the brain to provide information about both the structure and function of the brain [4, 28, 43]. The inverse source scattering problem has also attracted much research in the community of antenna design and synthesis [41]. A variety of antenna-embedding materials or substrates, including nonmagnetic dielectrics, magneto-dielectrics, and double negative meta-materials are of great interest.

Driven by these significant applications, the inverse source scattering problems have continuously received much attention and have been extensively studied by many researchers. There are a lot of available mathematical and numerical results, especially for the acoustic waves or the Helmholtz equation [1, 7, 14, 25, 27, 45]. In general, the inverse source problem does not have a unique solution due to the existence of non-radiating sources [9, 18, 26, 30]. Some addition constraint or information is needed in order to obtain a unique solution, such as to seek the minimum energy solution which represents the pseudo-inverse solution for the inverse problem. For electromagnetic waves, Ammari et al. showed uniqueness and presented an inversion scheme in [4] to reconstruct dipole sources based on a low-frequency asymptotic analysis of the time-harmonic Maxwell equations. In [3], Albanese and Monk discussed uniqueness and non-uniqueness of the inverse source problems for Maxwell's equations. Computationally, a more serious issue is the lack of stability, i.e., a small variation in the measured data may lead to a huge error in the reconstruction. Recently, it has been realized that the use of multi-frequency data can overcome the difficulties of non-uniqueness and instability which are encountered at a single frequency. In [15], Bao et al. initialized the mathematical study on the

2010 Mathematics Subject Classification. 78A46, 65C30.

Key words and phrases. Inverse source problem, Helmholtz equation, elastic wave equation, Gaussian random function, uniqueness. 
stability of the inverse source problem for the Helmholtz equation by using multi-frequency data. Since then, the increasing stability has become an interesting research topic in the study of inverse source problems [16, 20, 38]. We refer to [13] for a topic review on solving general inverse scattering problems with multi-frequencies.

Recently, the elastic wave scattering problems have received ever increasing attention for their important applications in may scientific areas such as geophysics and seismology [5, 21, 35, 42, 44]. However, the inverse source problem is much less studied for the elastic waves. The elastic wave equation is challenging due to the coexistence of compressional and shear waves that have different wavenumbers. Consequently, the Green tensor of the Navier equation has a more complicated expression than the Green function of the Helmholtz equation does. A more sophisticated analysis is required.

In many applications the source and hence the radiating field may not be deterministic but rather are modeled by random processes [8]. Therefore, their governing equations are stochastic differential equations. Although the deterministic counterparts have been well studied, little is known for the stochastic inverse problems due to randomness and uncertainties. A uniqueness result may be found in 24] for an inverse random source problem. It was shown that the autocorrelation function of the random source was uniquely determined by the auto-correlation function of the radiated field. Recently, effective mathematical models and efficient computational methods have been developed in [10 12, 17, 36, 37] for inverse random source scattering problems, where the stochastic wave equations are considered and the random sources are assumed to be driven by additive white noise. The inverse problems are formulated to determine the statistical properties, such as the mean and variance, of the random source from the boundary measurement of the wave field at multiple frequencies. The method requires to know the expectation of the scattering data. By the strong law of large numbers, the expectation has to be approximated by taking fairly large number of realizations of the measurement. We refer to [34] for statistical inversion theory on general random inverse problems.

In this paper, we consider a new model for the random source. A unified theory is developed on both of the direct and inverse scattering problems for the time-harmonic acoustic and elastic wave equations. The source is assumed to be a generalized Gaussian random function which is supported in a bounded domain $D \subset \mathbb{R}^{d}, d=2$ or 3 . In addition, we assume that the covariance of the random source is described by a pseudo-differential operator with the principle symbol given by $\phi(x)|\xi|^{-m}, m \in\left[d, d+\frac{1}{2}\right)$, where $\phi$ is a smooth non-negative function supported on $D$ and is called the micro-correlation strength of the source. This large class of random fields includes stochastic processed like the fractional Brownian motion and Markov field [40]. In fact, when $m \in\left[d, d+\frac{1}{2}\right.$ ), we can only ensure that the source belongs to a Sobolev space with negative smoothness index almost surely. Hence, the direct scattering problem requires a careful analysis since the source is a so-called rough field. In this work, we establish the well-posedness of the direct scattering problems for both wave equations with such rough sources. The inverse scattering problem aims at reconstructing the micro-correlation strength of the source $\phi$ from the scattered field measured in a bounded domain $U$ where $\bar{U} \cap \bar{D}=\emptyset$. For a single realization of the random source, we measure the amplitude of the scattering field averaged over the frequency band in a bounded and simply connected domain. Combining harmonic and microlocal analysis, we show that: for acoustic waves, the micro-correlation strength function $\phi$ can be recovered by these measurements; For elastic waves, note that the source is a vector, if the components of the random source are independent and the principle symbol of the pseudo-differential operator of each component coincides, thus, the micro-correlation strength function $\phi$ can be determined uniquely by these measurements.

This work is motivated by [19]40], where an inverse problem was considered for the two-dimensional random Schrödinger equation. The potential function in the Schrödinger equation was assumed to be a Gaussian random function with a pseudo-differential operator describing its covariance. It was shown that the principle symbol of the covariance operator can be determined uniquely by the 
backscattered field, generated by a single realization of the random potential and a point source as the incident field. A closely related problem can be found in [31. The authors considered the uniqueness for an inverse acoustic scattering problem in a half-space with an impedance boundary condition, where the impedance coefficient was assumed to a Gaussian random function whose covariance operator is a pseudo-differential operator.

The paper is organized as follows. In Section 2, we introduce some commonly used Sobolev spaces, give a precise mathematical description of the generalized Gaussian random function, and present several lemmas on rough fields and random variables. Section 3 is devoted to the study of the acoustic wave equation in the two- and three-dimensional cases. The well-posedness of the direct problems are examined. The uniqueness of the inverse problem are achieved. Section 4 addresses the two- and three-dimensional elastic wave equations. Analogous results are obtained. The direct problem is shown to have a unique solution and the inverse problem is proved to have the uniqueness to recover the principle symbol of the covariance operator for the random source. This paper is concluded with some general remarks in Section 5.

\section{Preliminaries}

In this section, we introduce some necessary notation such as Sobolev spaces and generalized Gaussian random functions which are used throughout the paper.

2.1. Sobolev spaces. Let $\mathbb{R}^{d}$ be the $d$-dimensional space, where $d=2$ or 3 . Denote by $C_{0}^{\infty}\left(\mathbb{R}^{d}\right)$ the set of smooth functions with compact support and by $\mathcal{D}^{\prime}\left(\mathbb{R}^{d}\right)$ the set of generalized (distributional) functions. For $1<p<\infty, s \in \mathbb{R}$, the Sobolev space $H^{s, p}\left(\mathbb{R}^{d}\right)$ is defined by

$$
H^{s, p}\left(\mathbb{R}^{d}\right)=\left\{h=(I-\Delta)^{-\frac{s}{2}} g: g \in L^{p}\left(\mathbb{R}^{d}\right)\right\},
$$

which has the norm

$$
\|h\|_{H^{s, p}\left(\mathbb{R}^{d}\right)}=\left\|(I-\Delta)^{\frac{s}{2}} h\right\|_{L^{p}\left(\mathbb{R}^{d}\right)} .
$$

With the definition of Sobolev spaces in the whole space, we can define the Sobolev spaces $H^{s, p}(V)$ for any Lipschitz domain $V \subset \mathbb{R}^{d}$ as the restrictions to $V$ of the elements in $H^{s, p}\left(\mathbb{R}^{d}\right)$. The norm is defined by

$$
\|h\|_{H^{s, p}(V)}=\inf \left\{\|g\|_{H^{s, p}\left(\mathbb{R}^{d}\right)}:\left.g\right|_{V}=h\right\} .
$$

According to [33], for $s \in \mathbb{R}$ and $1<p<\infty$, we can define $H_{0}^{s, p}(V)$ as the space of all distributions $h \in H^{s, p}\left(\mathbb{R}^{d}\right)$ such that supp $\subset \subset \bar{V}$ and the the norm is defined by

$$
\|h\|_{H_{0}^{s, p}(V)}=\|h\|_{H^{s, p}\left(\mathbb{R}^{d}\right)} .
$$

It is known that $C_{0}^{\infty}(V)$ is dense in $H_{0}^{s, p}(V)$ for any $1<p<\infty, s \in \mathbb{R} ; C_{0}^{\infty}(V)$ is dense in $H^{s, p}(V)$ for any $1<p<\infty, s \leq 0 ; C^{\infty}(\bar{V})$ is dense in $H^{s, p}(V)$ for any $1<p<\infty, s \in \mathbb{R}$. Additionally, by [33, Propositions 2.4 and 2.9], for any $s \in \mathbb{R}, p, q \in(1, \infty)$ satisfying $\frac{1}{p}+\frac{1}{q}=1$, we have

$$
H_{0}^{-s, q}(V)=\left(H^{s, p}(V)\right)^{\prime} \quad \text { and } \quad H^{-s, q}(V)=\left(H_{0}^{s, p}(V)\right)^{\prime},
$$

where the prime denotes the dual space.

2.2. Generalized Gaussian random functions. In this subsection, we provide a precise mathematical description of the generalized Gaussian random function. Let $(\Omega, \mathcal{F}, \mathcal{P})$ be a complete probability space. The function $q$ is said to be a generalized Gaussian random function if $q: \Omega \rightarrow \mathcal{D}^{\prime}\left(\mathbb{R}^{d}\right)$ is a measurable map such that for every $\hat{\omega} \in \Omega$, the mapping $\hat{\omega} \in \Omega \longmapsto\langle q(\hat{\omega}), \psi\rangle$ is a Gaussian random variable for all $\psi \in C_{0}^{\infty}\left(\mathbb{R}^{d}\right)$. The expectation and the covariance of the generalized Gaussian random function $q$ can be defined by

$$
\begin{aligned}
\mathbb{E} q: \psi \in C_{0}^{\infty}\left(\mathbb{R}^{d}\right) & \longmapsto \mathbb{E}\langle q, \psi\rangle \in \mathbb{R}, \\
\operatorname{Cov} q:\left(\psi_{1}, \psi_{2}\right) \in C_{0}^{\infty}\left(\mathbb{R}^{d}\right)^{2} & \longmapsto \operatorname{Cov}\left(\left\langle q, \psi_{1}\right\rangle,\left\langle q, \psi_{2}\right\rangle\right) \in \mathbb{R},
\end{aligned}
$$


where $\mathbb{E}\langle q, \psi\rangle$ denotes the expectation of $\langle q, \psi\rangle$ and

$$
\operatorname{Cov}\left(\left\langle q, \psi_{1}\right\rangle,\left\langle q, \psi_{2}\right\rangle\right)=\mathbb{E}\left(\left(\left\langle q, \psi_{1}\right\rangle-\mathbb{E}\left\langle q, \psi_{1}\right\rangle\right)\left(\left\langle q, \psi_{2}\right\rangle-\mathbb{E}\left\langle q, \psi_{2}\right\rangle\right)\right)
$$

denotes the covariance of $\left\langle q, \psi_{1}\right\rangle$ and $\left\langle q, \psi_{2}\right\rangle$. The covariance operator $C_{q}: C_{0}^{\infty}\left(\mathbb{R}^{d}\right) \rightarrow \mathcal{D}^{\prime}\left(\mathbb{R}^{d}\right)$ is defined by

$$
\left\langle C_{q} \psi_{1}, \psi_{2}\right\rangle=\operatorname{Cov}\left(\left\langle q, \psi_{1}\right\rangle,\left\langle q, \psi_{2}\right\rangle\right)=\mathbb{E}\left(\left\langle q-\mathbb{E} q, \psi_{1}\right\rangle\left\langle q-\mathbb{E} q, \psi_{2}\right\rangle\right) .
$$

Let $k_{q}(x, y)$ be the Schwartz kernel of the covariance operator $C_{q}$. We also call $k_{q}(x, y)$ the covariance function of $q$. Thus, (2.1) means that

$$
k_{q}(x, y)=\mathbb{E}((q(x)-\mathbb{E} q(x))(q(y)-\mathbb{E} q(y)))
$$

in the sense of generalized functions.

In this paper, we assume that each component of the external source is a generalized, microlocally isotropic Gaussian random function. For this end, let $D \subset \mathbb{R}^{d}$ be a bounded and simply connected domain. We introduce the following definition.

Definition 2.1. A generalized Gaussian random function $q$ on $\mathbb{R}^{d}$ is called microlocally isotropic of order $m$ in $D$, if the realizations of $q$ are almost surely supported in the domain $D$ and its covariance operator $C_{q}$ is a classical pseudo-differential operator having the principal symbol $\phi(x)|\xi|^{-m}$, where $\phi \in C_{0}^{\infty}\left(\mathbb{R}^{d}\right)$, supp $\phi \subset D$ and $\phi(x) \geq 0$ for all $x \in \mathbb{R}^{d}$.

In particular, we are interested in the case $m \in\left[d, d+\frac{1}{2}\right.$ ), which corresponds to rough fields. Now we introduce three lemmas and give an assumption which will be used in subsequent analysis.

Lemma 2.2. Let $f$ be a generalized and microlocally isotropic Gaussian random function of order $m$ in $D$. If $m=d$, then $f \in H^{-\varepsilon, p}(D)$ almost surely for all $\varepsilon>0,1<p<\infty$. If $m \in\left(d, d+\frac{1}{2}\right)$, then $f \in C^{\alpha}(D)$ almost surely for all $\alpha \in\left(0, \frac{m-d}{2}\right)$.

Lemma 2.3. Let $X$ and $Y$ be two zero-mean random variables such that the pair $(X, Y)$ is a Gaussian random vector. Then we have

$$
\mathbb{E}\left(\left(X^{2}-\mathbb{E} X^{2}\right)\left(Y^{2}-\mathbb{E} Y^{2}\right)\right)=2(\mathbb{E} X Y)^{2} .
$$

Lemma 2.4. Let $X_{t}, t \geq 0$ be a real valued stochastic process with a continuous path of zero mean, i.e., $\mathbb{E} X_{t}=0$. Assume that for some constants $c>0$ and $\beta>0$ such that the condition

$$
\left|\mathbb{E}\left(X_{t} X_{t+r}\right)\right| \leq c(1+r)^{-\beta}
$$

holds for all $t, r \geq 0$. Then

$$
\lim _{Q \rightarrow \infty} \frac{1}{Q} \int_{1}^{Q} X_{t} \mathrm{~d} t=0
$$

almost surely.

Lemma 2.2 is a direct consequence of Theorem 2 in [40]. Lemma 2.3 is shown in [19] as Lemma 4.2. The ergodic result of Lemma 2.4 is an immediate corollary of [23, p. 94]. To establish the main results, we need the following assumption.

Assumption A: The external source $f$ is assumed to have a compact support $D \subset \mathbb{R}^{d}$. Let $U \subset \mathbb{R}^{d} \backslash \bar{D}$ be the measurement domain of the wave field. We assume that $D$ and $U$ are two bounded and simply connected domains and there is a positive distance between $D$ and $U$. 


\section{Acoustic waves}

This section addresses the direct and inverse source scattering problems for the Helmholtz equation in two- and three-dimensional space. The external source is assumed to be a generalized Gaussian random function whose covariance operator is a classical pseudo-differential operator. The direct problem is shown to have a unique solution. For the inverse problem, we show that the principle symbol of the covariance operator can be determined uniquely by the scattered field obtained from a single realization of the random source.

3.1. The direct scattering problem. Consider the Helmholtz equation in a homogeneous medium

$$
\Delta u+\kappa^{2} u=f \quad \text { in } \mathbb{R}^{d},
$$

where $\kappa>0$ is the wavenumber, $u$ is the wave field, and $f$ is a generalized Gaussian random function. Note that $u$ is a random field since $f$ is a random function. To ensure the uniqueness of the solution for (3.1), the usual Sommerfeld radiation condition is imposed

$$
\lim _{r \rightarrow \infty} r^{\frac{d-1}{2}}\left(\partial_{r} u-\mathrm{i} \kappa u\right)=0, \quad r=|x|,
$$

uniformly for all directions $\hat{x}=x /|x|$. In addition, the external source function $f$ satisfies the following assumption.

Assumption B: The generalized Gaussian random field $f$ is microlocally isotropic of order $m$ in $D$, where $m \in\left[d, d+\frac{1}{2}\right)$. The principle symbol of its covariance operator $C_{f}$ is $\phi(x)|\xi|^{-m}$ with $\phi \in C_{0}^{\infty}(D)$ and $\phi \geq 0$. Moreover, the mean value of $f$ is zero, i.e., $\mathbb{E}(f)=0$.

By Lemma 2.2, the random source $f(\hat{\omega})$ belongs with probability one to the Sobolev space $H^{-\varepsilon, p}(D)$ for all $\varepsilon>0,1<p<\infty$. Hence it suffices to show that the direct scattering problem is well-posed when $f$ is a deterministic non-smooth function in $H^{-\varepsilon, p}(D)$.

First, we show some regularity results of the fundamental solution. These results play an important role in the proof of the well-posedness. Let $\Phi_{d}(x, y, \kappa)$ be the fundamental solution for the two- and three-dimensional Helmholtz equation. Explicitly, we have

$$
\Phi_{2}(x, y, \kappa)=\frac{\mathrm{i}}{4} H_{0}^{(1)}(\kappa|x-y|), \quad \Phi_{3}(x, y, \kappa)=\frac{1}{4 \pi} \frac{e^{\mathrm{i} \kappa|x-y|}}{|x-y|}
$$

where $H_{0}^{(1)}$ is the Hankel function of the first kind with order zero. We shall study the asymptotic properties of the fundamental solutions and their derivatives when $x$ is close to $y$. For the twodimensional case, we recall that

$$
H_{n}^{(1)}(t)=J_{n}(t)+\mathrm{i} Y_{n}(t),
$$

where $J_{n}$ and $Y_{n}$ are the Bessel functions of the first and second kind with order $n$, respectively. They admit the following expansions

$$
\begin{aligned}
J_{n}(t)= & \sum_{p=0}^{\infty} \frac{(-1)^{p}}{p !(n+p) !}\left(\frac{t}{2}\right)^{n+2 p} \\
Y_{n}(t)= & \frac{2}{\pi}\left\{\ln \frac{t}{2}+\gamma\right\} J_{n}(t)-\frac{1}{\pi} \sum_{p=0}^{n-1} \frac{(n-1-p) !}{p !}\left(\frac{2}{t}\right)^{n-2 p} \\
& -\frac{1}{\pi} \sum_{p=0}^{\infty} \frac{(-1)^{p}}{p !(n+p) !}\left(\frac{t}{2}\right)^{n+2 p}\{\psi(p+n)+\psi(p)\},
\end{aligned}
$$


where $\gamma:=\lim _{p \rightarrow \infty}\left\{\sum_{j=1}^{p} j^{-1}-\ln p\right\}$ denotes the Euler constant, $\psi(0)=0, \psi(p)=\sum_{j=1}^{p} j^{-1}$, and the finite sum in (3.6) is set to be zero for $n=0$. Using (3.4)-(3.6), we may verify that

$$
\begin{aligned}
& H_{0}^{(1)}(t)=\frac{2 \mathrm{i}}{\pi} \ln \frac{t}{2}+\left(1+\frac{2 \mathrm{i}}{\pi} \gamma\right)+O\left(t^{2} \ln \frac{t}{2}\right), \\
& H_{1}^{(1)}(t)=-\frac{2 \mathrm{i}}{\pi} \frac{1}{t}+\frac{\mathrm{i}}{\pi} t \ln \frac{t}{2}+\left(1+\frac{2 \mathrm{i}}{\pi} \gamma-\frac{\mathrm{i}}{\pi}\right) \frac{t}{2}+O\left(t^{3} \ln \frac{t}{2}\right) .
\end{aligned}
$$

Using the recurrence relations for the Hankel function of the first kind (see [39, Eq. (5.6.3)])

$$
\frac{\mathrm{d}}{\mathrm{d} t}\left[t^{-n} H_{n}^{(1)}(t)\right]=-t^{-n} H_{n+1}^{(1)}(t),
$$

we may show from (3.7)-(3.8) that

$$
\begin{aligned}
\Phi_{2}(x, y, \kappa) & =\frac{\mathrm{i}}{4} H_{0}^{(1)}(\kappa|x-y|) \\
& =-\frac{1}{2 \pi} \ln \frac{|x-y|}{2}+\left(\frac{\mathrm{i}}{4}-\frac{\gamma}{2 \pi}\right)+O\left(|x-y|^{2} \ln \frac{|x-y|}{2}\right), \\
\partial_{y_{i}} \Phi_{2}(x, y, \kappa) & =-\frac{\kappa \mathrm{i}}{4}\left(y_{i}-x_{i}\right) \frac{H_{1}^{(1)}(\kappa|x-y|)}{|x-y|} \\
& =-\frac{1}{2 \pi} \frac{y_{i}-x_{i}}{|x-y|^{2}}+O\left(\left(y_{i}-x_{i}\right) \ln \frac{|x-y|}{2}\right) .
\end{aligned}
$$

For the three-dimensional case, a simple calculation yields that

$$
\begin{aligned}
\Phi_{3}(x, y, \kappa) & =\frac{e^{\mathrm{i} \kappa|x-y|}}{4 \pi|x-y|}, \\
\partial_{y_{i}} \Phi_{3}(x, y, \kappa) & =\frac{\left(y_{i}-x_{i}\right)}{4 \pi|x-y|^{3}} e^{\mathrm{i} \kappa|x-y|}(\mathrm{i} \kappa|x-y|-1) .
\end{aligned}
$$

Lemma 3.1. Given any $x \in \mathbb{R}^{d}$, we have $\Phi_{2}(x, \cdot, \kappa) \in L_{\mathrm{loc}}^{2}\left(\mathbb{R}^{2}\right) \cap H_{\mathrm{loc}}^{1, p}\left(\mathbb{R}^{2}\right)$ for any $p \in(1,2)$ and $\Phi_{3}(x, \cdot, \kappa) \in L_{\mathrm{loc}}^{2}\left(\mathbb{R}^{3}\right) \cap H_{\mathrm{loc}}^{1, p}\left(\mathbb{R}^{3}\right)$ for any $p \in\left(1, \frac{3}{2}\right)$.

Proof. For any fixed $x \in \mathbb{R}^{d}$, let $V \subset \mathbb{R}^{d}$ be a bounded domain containing $x$. Denote $\rho:=\sup _{y \in V}|x-y|$, then we have $V \subset B_{\rho}(x)$.

For $d=2$, by (3.10) and (3.11), it suffices to show that

$$
\ln \frac{|x-y|}{2} \in L^{2}(V), \quad \frac{y_{i}-x_{i}}{|x-y|^{2}} \in L^{p}(V), \quad \forall p \in(1,2) .
$$

A direct calculation yields

$$
\int_{V}\left|\ln \frac{|x-y|}{2}\right|^{2} \mathrm{~d} y \leq \int_{B_{\rho}(x)}\left|\ln \frac{|x-y|}{2}\right|^{2} \mathrm{~d} y \lesssim \int_{0}^{\rho} r\left|\ln \frac{r}{2}\right|^{2} \mathrm{~d} r<\infty
$$

and

$$
\int_{V}\left|\frac{y_{i}-x_{i}}{|x-y|^{2}}\right|^{p} \mathrm{~d} y \leq \int_{B_{\rho}(x)} \frac{1}{|x-y|^{p}} \mathrm{~d} y \lesssim \int_{0}^{\rho} r^{1-p} \mathrm{~d} r<\infty, \quad \forall p \in(1,2) .
$$

Hereafter, the notation $a \lesssim b$ means $a \leq C b$, where $C>0$ is a generic constant which may change step by step in the proofs. Thus, we conclude that $\Phi_{2}(x, \cdot, \kappa) \in L_{\text {loc }}^{2}\left(\mathbb{R}^{2}\right) \cap H_{\text {loc }}^{1, p}\left(\mathbb{R}^{2}\right)$ for any $p \in(1,2)$.

For $d=3$, from (3.12) and (3.13), it suffices to prove that

$$
\frac{e^{\mathrm{i} \kappa|x-y|}}{|x-y|} \in L^{2}(V), \quad e^{\mathrm{i} \kappa|x-y|} \frac{y_{i}-x_{i}}{|x-y|^{3}} \in L^{p}(V) \quad \forall p \in\left(1, \frac{3}{2}\right) .
$$


Similarly, we may have from a simple calculation that

$$
\int_{V}\left|\frac{e^{\mathrm{i} \kappa|x-y|}}{|x-y|}\right|^{2} \mathrm{~d} y \leq \int_{B_{\rho}(x)} \frac{1}{|x-y|^{2}} \mathrm{~d} y \lesssim \int_{0}^{\rho} 1 \mathrm{~d} r<\infty
$$

and

$$
\int_{V}\left|e^{\mathrm{i} \kappa|x-y|} \frac{y_{i}-x_{i}}{|x-y|^{3}}\right|^{p} \mathrm{~d} y \leq \int_{B_{\rho}(x)} \frac{1}{|x-y|^{2 p}} \mathrm{~d} y \lesssim \int_{0}^{\rho} r^{2-2 p} \mathrm{~d} r<\infty \quad \forall p \in\left(1, \frac{3}{2}\right),
$$

which show that $\Phi_{3}(x, \cdot, \kappa) \in L_{\text {loc }}^{2}\left(\mathbb{R}^{3}\right) \cap H_{\text {loc }}^{1, p}\left(\mathbb{R}^{3}\right)$ for any $p \in\left(1, \frac{3}{2}\right)$.

Let $V$ and $G$ be any two bounded domains in $\mathbb{R}^{d}$. By Lemma 3.1 and the Sobolev embedding theorem, we obtain that $\Phi_{d}(x, \cdot, \kappa) \in H^{s}(V)$ where $s \in(0,1)$ for $d=2$ and $s \in\left(0, \frac{1}{2}\right)$ for $d=3$. Hence, given $g \in H_{0}^{-s}(V)$, we can define the operator $H_{\kappa}$ in the dual sense by

$$
H_{\kappa} g(x)=\int_{V} \Phi_{d}(x, y, \kappa) g(y) \mathrm{d} y, \quad x \in G .
$$

Following the similar arguments in [22, Theorem 8.2], we may show the following regularity of the operator $H_{\kappa}$. The proof is omitted here for brevity.

Lemma 3.2. The operator $H_{\kappa}: H_{0}^{-s}(V) \rightarrow H^{s}(G)$ is bounded for $s \in(0,1)$ in two dimensions or for $s \in\left(0, \frac{1}{2}\right)$ in three dimensions.

Theorem 3.3. For some fixed $s \in\left(0,1-\frac{d}{6}\right)$, assume $1<p<\frac{2 d}{d+2(1-s)}$ and $\frac{1}{p}+\frac{1}{p^{\prime}}=1$, then the scattering problem (3.1)-(3.2) with the source $f \in H_{0}^{-1, p^{\prime}}(D)$ attains a unique solution $u \in H_{\mathrm{loc}}^{1, p}\left(\mathbb{R}^{d}\right)$, which can be represented by

$$
u(x, \kappa)=-\int_{D} \Phi_{d}(x, y, \kappa) f(y) \mathrm{d} y .
$$

Proof. It is clear that the scattering problem (3.1)-(3.2) with $f=0$ only has the zero solution. Hence the uniqueness follows. Now we focus on the existence. Since $s \in\left(0,1-\frac{d}{6}\right)$, a simple calculation shows that $1<\frac{2 d}{d+2(1-s)}<\frac{3}{2}$. By Lemma 3.1, we obtain that $\Phi_{d}(x, \cdot, \kappa) \in H_{\text {loc }}^{1, p}\left(\mathbb{R}^{d}\right)$. Since $\Delta u+\kappa^{2} u=f \in H_{0}^{-1, p^{\prime}}(D)$, we have in the sense of distribution that

$$
\int_{B_{r}}\left(\Delta u(y)+\kappa^{2} u(y)\right) \Phi_{d}(x, y, \kappa) \mathrm{d} y=\int_{B_{r}} \Phi_{d}(x, y, \kappa) f(y) \mathrm{d} y .
$$

Here $B_{r}=\left\{y \in \mathbb{R}^{d}:|y| \leq r\right\}$, where $r>0$ is sufficiently large such that $\bar{D} \subset B_{r}$. Denote by $S_{\mathrm{A}}$ the operator which acts on $u$ on the left-hand side of (3.15). For $\varphi \in C^{\infty}\left(\mathbb{R}^{d}\right)$, we have

$$
\begin{aligned}
& \left(S_{\mathrm{A}} \varphi\right)(x):=\int_{B_{r}}\left(\Delta \varphi(y)+\kappa^{2} \varphi(y)\right) \Phi_{d}(x, y, \kappa) \mathrm{d} y \\
= & \int_{B_{r} \backslash B_{\delta}(x)}\left(\Delta \varphi(y)+\kappa^{2} \varphi(y)\right) \Phi_{d}(x, y, \kappa) \mathrm{d} y+\int_{B_{\delta}(x)}\left(\Delta \varphi(y)+\kappa^{2} \varphi(y)\right) \Phi_{d}(x, y, \kappa) \mathrm{d} y \\
= & \int_{B_{r} \backslash B_{\delta}(x)}\left(\Delta \varphi(y) \Phi_{d}(x, y, \kappa)-\varphi(y) \Delta \Phi_{d}(x, y, \kappa)\right) \mathrm{d} y+\int_{B_{\delta}(x)}\left(\Delta \varphi(y)+\kappa^{2} \varphi(y)\right) \Phi_{d}(x, y, \kappa) \mathrm{d} y \\
= & \int_{B_{\delta}(x)}\left(\Delta \varphi(y)+\kappa^{2} \varphi(y)\right) \Phi_{d}(x, y, \kappa) \mathrm{d} y+\int_{\partial B_{r}}\left(\frac{\partial \varphi(y)}{\partial \nu(y)} \Phi_{d}(x, y, \kappa)-\varphi(y) \frac{\partial \Phi_{d}(x, y, \kappa)}{\partial \nu(y)}\right) \mathrm{d} s(y) \\
& +\int_{\partial B_{\delta}(x)}\left(\frac{\partial \varphi(y)}{\partial \nu(y)} \Phi_{d}(x, y, \kappa)-\varphi(y) \frac{\partial \Phi_{d}(x, y, \kappa)}{\partial \nu(y)}\right) \mathrm{d} s(y),
\end{aligned}
$$


where $\delta>0$ is a sufficiently small number, and $\nu(y)$ denotes the unit normal which directs to the exterior of $B_{r}$ for $y \in \partial B_{r}$ and directs to the interior of $B_{\delta}(x)$ for $y \in \partial B_{\delta}(x)$. Using the mean value theorem, we get

$$
\lim _{\delta \rightarrow 0} \int_{\partial B_{\delta}(x)}\left(\frac{\partial \varphi(y)}{\partial \nu(y)} \Phi_{d}(x, y, \kappa)-\varphi(y) \frac{\partial \Phi_{d}(x, y, \kappa)}{\partial \nu(y)}\right) \mathrm{d} s(y)=-\varphi(x)
$$

and

$$
\lim _{\delta \rightarrow 0} \int_{B_{\delta}(x)}\left(\Delta \varphi(y)+\kappa^{2} \varphi(y)\right) \Phi_{d}(x, y, \kappa) \mathrm{d} y=0 .
$$

Combining the above equations gives that

$$
\left(S_{\mathrm{A}} \varphi\right)(x)=-\varphi(x)+\int_{\partial B_{r}}\left(\frac{\partial \varphi(y)}{\partial \nu(y)} \Phi_{d}(x, y, \kappa)-\varphi(y) \frac{\partial \Phi_{d}(x, y, \kappa)}{\partial \nu(y)}\right) \mathrm{d} s(y),
$$

which implies

$$
\left(S_{\mathrm{A}} u\right)(x)=-u(x)+\int_{\partial B_{r}}\left(\frac{\partial u(y)}{\partial \nu(y)} \Phi_{d}(x, y, \kappa)-u(y) \frac{\partial \Phi_{d}(x, y, \kappa)}{\partial \nu(y)}\right) \mathrm{d} s(y) .
$$

Since both $u$ and $\Phi_{d}$ satisfy the Sommerfeld radiation condition, we have

$$
\lim _{r \rightarrow \infty} \int_{\partial B_{r}}\left(\frac{\partial u(y)}{\partial \nu(y)} \Phi_{d}(x, y, \kappa)-u(y) \frac{\partial \Phi_{d}(x, y, \kappa)}{\partial \nu(y)}\right) \mathrm{d} s(y)=0 .
$$

Therefore

$$
u(x, \kappa)=-\int_{D} \Phi_{d}(x, y, \kappa) f(y) \mathrm{d} y=-H_{\kappa} f(x) .
$$

Next is to show that $u \in H_{\text {loc }}^{1, p}\left(\mathbb{R}^{d}\right)$. From Lemma 3.2, we have that the operator $H_{\kappa}: H_{0}^{-s}(D) \rightarrow$ $H_{\mathrm{loc}}^{s}\left(\mathbb{R}^{d}\right)$ for $s \in\left(0,1-\frac{d}{6}\right)$ is bounded. The assumption $1<p<\frac{2 d}{d+2(1-s)}$ implies that $\frac{1}{2}+\frac{1-s}{d}<\frac{1}{p}<1$ which yields $\frac{1}{2}-\frac{s}{d}<\frac{1}{p}-\frac{1}{d}$. Thus, the Sobolev embedding theorem implies that $H^{s}(D)$ is embedded into $H^{1, p}(D)$ and $H_{0}^{-1, p^{\prime}}(D)$ is embedded into $H_{0}^{-s}(D)$. Thus, the operator $H_{\kappa}: H_{0}^{-1, p^{\prime}}(D) \rightarrow$ $H_{\text {loc }}^{1, p}\left(\mathbb{R}^{d}\right)$ is bounded, which completes the proof.

3.2. The two-dimensional case. First we discuss the two-dimensional case and show that the function $\phi$ in the principle symbol can be uniquely determined by the scattered field obtained from a single realization of the random source $f$. Let us begin with the asymptotic of the Hankel function $H_{n}^{(1)}$ with a large argument. By [6, Eqs. (9.2.7)-(9.2.10)] and [39, Eqs.(5.11.4)], we have:

$$
H_{n}^{(1)}(z)=\sqrt{\frac{1}{z}} e^{\mathrm{i}\left(z-\left(\frac{n}{2}+\frac{1}{4}\right) \pi\right)}\left(\sum_{j=0}^{N} a_{j}^{(n)} z^{-j}+O\left(|z|^{-N-1}\right)\right), \quad \text { for }|\arg z| \leq \pi-\delta,
$$

for large $|z|$, where $\delta$ is an arbitrarily small positive number and the coefficients $a_{j}^{(n)}=(-2 \mathrm{i})^{j} \sqrt{\frac{2}{\pi}}(n, j)$ with

$$
(n, j)=\frac{\left(4 n^{2}-1\right)\left(4 n^{2}-3^{2}\right) \cdots\left(4 n^{2}-(2 j-1)^{2}\right)}{2^{2 j} j !} \quad \text { and } \quad(n, 0)=1 .
$$

Using the first $N$ terms in the asymptotic of $H_{n}^{(1)}(\kappa|z|)$, we define

$$
H_{n, N}^{(1)}(\kappa|z|):=\sqrt{\frac{1}{\kappa|z|}} e^{\mathrm{i}\left(\kappa|z|-\left(\frac{n}{2}+\frac{1}{4}\right) \pi\right)} \sum_{j=0}^{N} a_{j}^{(n)}\left(\frac{1}{\kappa|z|}\right)^{j}
$$


It is easy to show from (3.16) that

$$
\left|H_{n}^{(1)}(\kappa|z|)-H_{n, N}^{(1)}(\kappa|z|)\right| \leq c\left(\frac{1}{\kappa|z|}\right)^{N+\frac{3}{2}} .
$$

Using (3.17), we define $\tilde{u}(x, \kappa)$ as

$$
\tilde{u}(x, \kappa):=-\frac{\mathrm{i}}{4} \int_{\mathbb{R}^{2}} H_{0,2}^{(1)}(\kappa|x-y|) f(y) \mathrm{d} y .
$$

Lemma 3.4. The random variable $u(x, \kappa)-\tilde{u}(x, \kappa)$ satisfies almost surely the condition

$$
|u(x, \kappa)-\tilde{u}(x, \kappa)| \leq c \kappa^{-\frac{7}{2}}, \quad x \in U,
$$

where the constant $c$ depends only on $L^{2}(D)$-norm of $f$.

Proof. Noting Assumption A, we know that there exists a positive constant $M$ such that $|x-y| \geq M$ holds for all $x \in U$ and $y \in D$. By (3.14), (3.18), and (3.19), we have for $x \in U$ that

$$
\begin{aligned}
|u(x, \kappa)-\tilde{u}(x, \kappa)| & =\left|\frac{\mathrm{i}}{4} \int_{D}\left[H_{0}^{(1)}(\kappa|x-y|)-H_{0,2}^{(1)}(\kappa|x-y|)\right] f(y) d y\right| \\
& \lesssim\left\|H_{0}^{(1)}(\kappa|x-\cdot|)-H_{0,2}^{(1)}(\kappa|x-\cdot|)\right\|_{H^{1, p}(D)}\|f\|_{H_{0}^{-1, p^{\prime}}(D)} \\
& \leq c \kappa^{-\frac{7}{2}}
\end{aligned}
$$

where the constant $c$ depends only on $H_{0}^{-1, p}(D)$-norm of $f$.

Now we are in the position to compute the covariance of $\tilde{u}(x, \kappa)$. Using (3.17) and (3.19), we have from a direct calculation that

$$
\mathbb{E}\left(\tilde{u}\left(x, \kappa_{1}\right) \overline{\tilde{u}\left(x, \kappa_{2}\right)}\right)=\frac{1}{16} \sum_{j_{1}, j_{2}=0}^{2} \frac{a_{j_{1}}^{(0)} \overline{a_{j_{2}}^{(0)}}}{\kappa_{1}^{j_{1}+\frac{1}{2}} \kappa_{2}^{j_{2}+\frac{1}{2}}} \int_{\mathbb{R}^{4}} \frac{e^{\mathrm{i}\left(\kappa_{1}|x-y|-\kappa_{2}|x-z|\right)}}{|x-y|^{j_{1}+\frac{1}{2}}|x-z|^{j_{2}+\frac{1}{2}}} \mathbb{E}(f(y) f(z)) \mathrm{d} y \mathrm{~d} z .
$$

From (3.20), it is easy to see that $\mathbb{E}\left(\tilde{u}\left(x, \kappa_{1}\right) \overline{\tilde{u}\left(x, \kappa_{2}\right)}\right)$ is a linear combination of the following integral

$$
I\left(x, \kappa_{1}, \kappa_{2}\right):=\frac{1}{\kappa_{1}^{l_{1}} \kappa_{2}^{l_{2}}} \int_{\mathbb{R}^{2 d}} e^{\mathrm{i}\left(c_{1} \kappa_{1}|x-y|-c_{2} \kappa_{2}|x-z|\right)} K(x, y, z) \mathbb{E}(q(y) q(z)) \mathrm{d} y \mathrm{~d} z
$$

where

$$
K(x, y, z):=\frac{\left(x_{1}-y_{1}\right)^{m_{1}} \cdots\left(x_{d}-y_{d}\right)^{m_{d}}\left(x_{1}-z_{1}\right)^{n_{1}} \cdots\left(x_{d}-z_{d}\right)^{n_{d}}}{|x-y|^{p_{1}}|x-z|^{p_{2}}} .
$$

Here $q$ stands for a generalized Gaussian random function satisfying Assumption B, and $l_{1}, l_{2}, c_{1}$, $c_{2}, m_{1}, \ldots, n_{d}, p_{1}, p_{2}$ are nonnegative constants.

Lemma 3.5. For $\kappa_{1}, \kappa_{2} \geq 1$, the estimates

$$
\begin{aligned}
\left|I\left(x, \kappa_{1}, \kappa_{2}\right)\right| & \leq c_{n}\left(\kappa_{1}+\kappa_{2}\right)^{-\left(m+2 \min \left(l_{1}, l_{2}\right)\right)}\left(1+\left|\kappa_{1}-\kappa_{2}\right|\right)^{-n}, \\
\left|\mathbb{E}\left(\tilde{u}\left(x, \kappa_{1}\right) \tilde{u}\left(x, \kappa_{2}\right)\right)\right| & \leq c_{n}\left(\kappa_{1}+\kappa_{2}\right)^{-n}\left(1+\left|\kappa_{1}-\kappa_{2}\right|\right)^{-m}
\end{aligned}
$$

holds uniformly for $x \in U$, where $n \in \mathbb{N}$ is arbitrary.

Proof. To estimate the integral $I\left(x, \kappa_{1}, \kappa_{2}\right)$, we introduce the multiple coordinate transformation that allows to use the microlocal methods in our analysis. Noting $\mathbb{E} q=0$ and (2.2), we conclude that the correlation function $\mathbb{E}(q(y) q(z))$ is the Schwartz kernel of a pseudo-differential operator $C_{q}$ with a classical symbol $\sigma(y, \xi) \in S_{1,0}^{-m}\left(\mathbb{R}^{d} \times \mathbb{R}^{d}\right)$ which is defined by

$$
S_{1,0}^{-m}\left(\mathbb{R}^{d} \times \mathbb{R}^{d}\right):=\left\{a(x, \xi) \in C^{\infty}\left(\mathbb{R}^{d} \times \mathbb{R}^{d}\right):\left|\partial_{\xi}^{\alpha} \partial_{x}^{\beta} a(x, \xi)\right| \leq C_{\alpha, \beta}(1+|\xi|)^{-m-|\alpha|}\right\} .
$$


Here $\alpha, \beta$ are multiple indices, $|\alpha|$ denotes the sum of its component. The principle symbol of $C_{q}$ is $\sigma^{p}(y, \xi)=\phi(y)|\xi|^{-m}$. The support of $\mathbb{E}(q(y) q(z))$ is contained in $D \times D$. We can write $\mathbb{E}(q(y) q(z))$ in terms of its symbol by

$$
\mathbb{E}(q(y) q(z))=(2 \pi)^{-d} \int_{\mathbb{R}^{d}} e^{\mathrm{i}(y-z) \cdot \xi} \sigma(y, \xi) \mathrm{d} \xi .
$$

In order to establish a uniform estimate with respect to the variable $x$, we extend the covariance function into the space $\mathbb{R}^{2 d} \times \mathbb{R}^{d}$, and define $B_{1}(y, z, x)=\mathbb{E}(q(y) q(z)) \theta(x)$ where $\theta(x) \in C_{0}^{\infty}\left(\mathbb{R}^{d}\right)$ equals to one in the domain $U$ and has its support outside of the domain $\bar{D}$. Thus, we have

$$
B_{1}(y, z, x)=(2 \pi)^{-d} \int_{\mathbb{R}^{d}} e^{\mathrm{i}(y-z) \cdot \xi} c_{1}(y, x, \xi) \mathrm{d} \xi,
$$

where $c_{1}(y, x, \xi)=\sigma(y, \xi) \theta(x) \in S_{1,0}^{-m}\left(\mathbb{R}^{2 d} \times \mathbb{R}^{d}\right)$ with a principle symbol

$$
c_{1}^{p}(y, x, \xi)=\phi(y)|\xi|^{-m} \theta(x) .
$$

To proceed the analysis, let us briefly revisit the conormal distributions of Höremainder type [29]. If $X \subset \mathbb{R}^{d}$ is an open set and $S \subset X$ is a smooth submanifold of $X$, we denote by $I(X ; S)$ the distributions in $\mathcal{D}^{\prime}(X)$ that are smooth in $X \backslash S$ and have a conormal singularity at $S$. In consequence, by (3.24), the correlation function $\mathbb{E}(q(y) q(z))$ is a conormal distribution in $\mathbb{R}^{2 d}$ of Höremainder type having conormal singularity on the surface $S_{1}=\left\{(y, z) \in \mathbb{R}^{2 d}: y-z=0\right\}$. Moreover, let $I_{\text {comp }}(X ; S)$ be the set of distributions supported in a compact subset of $X$. Let $\boldsymbol{D} \subset \mathbb{R}^{3 d}$ be an open set containing $D \times D \times \operatorname{supp}(\theta)$ so that $B_{1} \in I_{\text {comp }}\left(\mathbf{D} ; S_{1} \cap \mathbf{D}\right)$.

Define the first coordinate transformation $\eta: \mathbb{R}^{3 d} \rightarrow \mathbb{R}^{3 d}$ by

$$
(v, w, x)=\eta(y, z, x)=(y-z, y+z, x) .
$$

Substituting the coordinate transformation (3.25) into $B_{1}(y, z, x)$ gives

$$
B_{2}(v, w, x)=B_{1}\left(\eta^{-1}(v, w, x)\right)=(2 \pi)^{-d} \int_{\mathbb{R}^{d}} e^{\mathrm{i} v \cdot \xi} c_{1}\left(\frac{v+w}{2}, x, \xi\right) \mathrm{d} \xi,
$$

which means that $B_{2} \in I\left(\mathbb{R}^{3 d}, S_{2}\right)$ where $S_{2}:=\{(v, w, x): v=0\}$. Actually, $B_{2} \in I_{\text {comp }}\left(X_{2}, X_{2} \cap S_{2}\right)$ where $X_{2}:=\eta(\mathbf{D})$. To find out how the symbol transforms in the change of coordinates, we need to represent $c_{1}\left(\frac{v+w}{2}, x, \xi\right)$ with a symbol that does not depend on $v$. Using the representation theorem of conormal distribution [29, Lemma 18.2.1]), we obtain

$$
B_{2}(v, w, x)=(2 \pi)^{-d} \int_{\mathbb{R}^{d}} e^{\mathrm{i} v \cdot \xi} c_{2}(w, x, \xi) \mathrm{d} \xi
$$

where $c_{2}(w, x, \xi)$ has the asymptotic expansion

$$
\left.c_{2}(w, x, \xi) \sim \sum_{l=0}^{\infty} \frac{\left\langle-\mathrm{i} D_{v}, D_{\xi}\right\rangle^{l}}{l !} c_{1}\left(\frac{v+w}{2}, x, \xi\right)\right|_{v=0} \in S_{1,0}^{-m}\left(\mathbb{R}^{2 d} \times \mathbb{R}^{d}\right) .
$$

In particular, the principle symbol of $c_{2}(w, x, \xi)$ is

$$
c_{2}^{p}(w, x, \xi)=\left.\phi\left(\frac{v+w}{2}\right)|\xi|^{-m} \theta(x)\right|_{v=0} .
$$

We consider the phase of $I\left(x, \kappa_{1}, \kappa_{2}\right)$. A simple calculation shows that

$$
\begin{aligned}
c_{1} \kappa_{1}|x-y|-c_{2} \kappa_{2}|x-z|= & \left(c_{1} \kappa_{1}+c_{2} \kappa_{2}\right) \frac{|x-y|-|x-z|}{2} \\
& +\left(c_{1} \kappa_{1}-c_{2} \kappa_{2}\right) \frac{|x-y|+|x-z|}{2} .
\end{aligned}
$$


In the second set of coordinates, let $\frac{|x-y| \pm|x-z|}{2}$ play the role of two coordinates. We will do this change in two steps. First, for the two-dimensional case where $d=2$, we define $\tau_{1}: \mathbb{R}^{6} \rightarrow \mathbb{R}^{6}$ by

$$
\tau_{1}(y, z, x)=\left(E_{1}, E_{2}, x\right)
$$

where $E_{1}=\left(t_{1}, s_{1}\right)$ and $E_{2}=\left(t_{2}, s_{2}\right)$ with

$$
\begin{aligned}
t_{1} & =\frac{1}{2}|x-y|, \quad s_{1}=\frac{1}{2} \arcsin \left(\frac{y_{1}-x_{1}}{|x-y|}\right), \\
t_{2} & =\frac{1}{2}|x-z|, \quad s_{2}=\frac{1}{2} \arcsin \left(\frac{z_{1}-x_{1}}{|x-z|}\right) .
\end{aligned}
$$

For the three-dimensional case where $d=3$, we define $\tau_{1}: \mathbb{R}^{9} \rightarrow \mathbb{R}^{9}$ by

$$
\tau_{1}(y, z, x)=\left(E_{1}, E_{2}, x\right)
$$

where $E_{1}=\left(t_{1}, s_{1}, r_{1}\right)$ and $E_{2}=\left(t_{2}, s_{2}, r_{2}\right)$ with

$$
\begin{array}{rlrl}
t_{1}=\frac{1}{2}|x-y|, & s_{1}=\frac{1}{2} \arccos \left(\frac{y_{3}-x_{3}}{|x-y|}\right), & r_{1}=\frac{1}{2}|x-y| \arctan \left(\frac{y_{2}-x_{2}}{y_{1}-x_{1}}\right), \\
t_{2}=\frac{1}{2}|x-z|, \quad s_{2}=\frac{1}{2} \arccos \left(\frac{z_{3}-x_{3}}{|x-z|}\right), & r_{2}=\frac{1}{2}|x-z| \arctan \left(\frac{z_{2}-x_{2}}{z_{1}-x_{1}}\right) .
\end{array}
$$

Second, we define $\tau_{2}: \mathbb{R}^{3 d} \rightarrow \mathbb{R}^{3 d}$ by

$$
(g, h, x)=\tau_{2}\left(E_{1}, E_{2}, x\right)=\left(E_{1}-E_{2}, E_{1}+E_{2}, x\right) .
$$

Thus, combining the definitions of $\tau_{1}, \tau_{2}$ and (3.26) , we have

$$
c_{1} \kappa_{1}|x-y|-c_{2} \kappa_{2}|x-z|=\left(c_{1} \kappa_{1}+c_{2} \kappa_{2}\right) g \cdot e_{1}+\left(c_{1} \kappa_{1}-c_{2} \kappa_{2}\right) h \cdot e_{1},
$$

where $e_{1}=(1,0)$ for $d=2$, and $e_{1}=(1,0,0)$ for $d=3$. Now we denote $\tau=\tau_{2} \circ \tau_{1}: \mathbb{R}^{3 d} \rightarrow$ $\mathbb{R}^{3 d}$ with $\tau(y, z, x)=(g, h, x)$. We consider the transformation $\rho=\eta \circ \tau^{-1}: \mathbb{R}^{3 d} \rightarrow \mathbb{R}^{3 d}$ with $\rho(g, h, x)=(v, w, x)$. Let us decompose the coordinate transform $\rho$ into two parts $\rho=\left(\rho_{1}, \rho_{2}\right)$, the $\mathbb{R}^{d}$-valued function $\rho_{1}(g, h, x)=v$ and the $\mathbb{R}^{2 d}$-valued function $\rho_{2}(g, h, x)=(w, x)$. The Jacobian $J_{\rho}$ corresponding to the decomposing of the variables is given by

$$
J_{\rho}=\left[\begin{array}{cc}
\rho_{11}^{\prime} & \rho_{12}^{\prime} \\
\rho_{21}^{\prime} & \rho_{22}^{\prime}
\end{array}\right]=\left[\begin{array}{ll}
J_{g} \rho_{1} & J_{(h, x)} \rho_{1} \\
J_{g} \rho_{2} & J_{(h, x)} \rho_{2}
\end{array}\right] .
$$

By the definition of $\rho$, it is easy to see that $v=0$ if $g=0$. Hence we have $\rho_{1}(0, h, x)=0$ which implies $\rho_{12}^{\prime}(0, h, x)=0$.

Next we consider the pull-back distribution $B_{3}=B_{2} \circ \rho$. It follow from [29, Theorem 18.2.9] that we get a representation for $B_{3}$ :

$$
B_{3}(g, h, x)=(2 \pi)^{-d} \int_{\mathbb{R}^{d}} e^{\mathrm{i} g \cdot \xi} c_{3}(h, x, \xi) \mathrm{d} \xi,
$$

where $c_{3}(h, x, \xi) \in S_{1,0}^{-m}\left(\mathbb{R}^{2 d} \times \mathbb{R}^{d}\right)$ is a symbol satisfying

$$
c_{3}(h, x, \xi)=c_{2}\left(\rho_{2}(g, h, x),\left(\left(\rho_{11}^{\prime}(g, h, x)\right)^{-1}\right)^{T} \xi\right) \times\left.\left|\operatorname{det} \rho_{11}^{\prime}(g, h, x)\right|^{-1}\right|_{g=0}+r(h, x, \xi),
$$

where $r(h, x, \xi) \in S_{1,0}^{-m-1}\left(\mathbb{R}^{2 d} \times \mathbb{R}^{d}\right)$. The principle symbol of $c_{3}(h, x, \xi)$ is given by

$$
c_{3}^{p}(h, x, \xi)=\phi(y(g, h, x))\left|\left(\left(\rho_{11}^{\prime}(g, h, x)\right)^{-1}\right)^{T} \xi\right|^{-m} \theta(x) \times\left.\left|\operatorname{det} \rho_{11}^{\prime}(g, h, x)\right|^{-1}\right|_{g=0} .
$$

Let $X_{3}:=\tau(\mathbf{D})$ and $S_{3}:=\{(g, h, x): g=0\}$, we have $B_{3} \in I_{\text {comp }}\left(X_{3}, X_{3} \cap S_{3}\right)$. So we can write $I\left(x, \kappa_{1}, \kappa_{2}\right)$ in the following form

$$
I\left(x, \kappa_{1}, \kappa_{2}\right)=\frac{1}{\kappa_{1}^{l_{1}} \kappa_{2}^{l_{2}}} \int_{\mathbb{R}^{2 d}} e^{\mathrm{i}\left[\left(c_{1} \kappa_{1}+c_{2} \kappa_{2}\right) g \cdot e_{1}+\left(c_{1} \kappa_{1}-c_{2} \kappa_{2}\right) h \cdot e_{1}\right]} B_{3}(g, h, x) H(g, h, x) \mathrm{d} g \mathrm{~d} h,
$$


where

$$
H(g, h, x)=K(x, y, z) \operatorname{det}\left(\left(\tau^{-1}\right)^{\prime}(g, h, x)\right) .
$$

Here $y=y(g, h, x)$ and $z=z(g, h, x)$. Since $H$ is smooth in $X_{3}$ in all variables and $I\left(\mathbb{R}^{3 d}, S_{3}\right)$ is closed under multiplication with a smooth function, we conclude that $B_{3}(g, h, x) H(g, h, x) \in I\left(\mathbb{R}^{3 d}, S_{3}\right)$. Multiplying (3.27) by $H$, we arrive at

$$
B_{3}(g, h, x) H(g, h, x)=(2 \pi)^{-d} \int_{\mathbb{R}^{d}} e^{\mathrm{i} g \cdot \xi} c_{4}(h, x, \xi) \mathrm{d} \xi,
$$

where $c_{4}(h, x, \xi)$ has the asymptotic expansion

$$
\left.c_{4}(h, x, \xi) \sim \sum_{l=0}^{\infty} \frac{\left\langle-\mathrm{i} D_{g}, D_{\xi}\right\rangle^{l}}{l !}\left(c_{3}(h, x, \xi) H(g, h, x)\right)\right|_{g=0} .
$$

In particular, the principle symbol of $c_{4}(h, x, \xi)$ is given by

$$
c_{4}^{p}(h, x, \xi)=\left.\phi(y(g, h, x))\left|\left(\left(\rho_{11}^{\prime}(g, h, x)\right)^{-1}\right)^{T} \xi\right|^{-m} \theta(x)\left|\operatorname{det} \rho_{11}^{\prime}(g, h, x)\right|^{-1} H(g, h, x)\right|_{g=0} .
$$

Combining (3.29) and the Fourier inversion rule, we obtain

$$
B_{3}(g, h, x) H(g, h, x)=\left(\mathcal{F}^{-1} c_{4}\right)(h, x, g) .
$$

Substituting (3.31) into $I\left(x, \kappa_{1}, \kappa_{2}\right)$ gives

$$
\begin{aligned}
I\left(x, \kappa_{1}, \kappa_{2}\right) & =\frac{1}{\kappa_{1}^{l_{1}} \kappa_{2}^{l_{2}}} \int_{\mathbb{R}^{2 d}} e^{\mathrm{i}\left[\left(c_{1} \kappa_{1}+c_{2} \kappa_{2}\right) g \cdot e_{1}+\left(c_{1} \kappa_{1}-c_{2} \kappa_{2}\right) h \cdot e_{1}\right]}\left(\mathcal{F}^{-1} c_{4}\right)(h, x, g) \mathrm{d} g \mathrm{~d} h \\
& =\frac{1}{\kappa_{1}^{l_{1}} \kappa_{2}^{l_{2}}} \int_{\mathbb{R}^{d}} e^{\mathrm{i}\left(c_{1} \kappa_{1}-c_{2} \kappa_{2}\right) h \cdot e_{1}} c_{4}\left(h, x,-\left(c_{1} \kappa_{1}+c_{2} \kappa_{2}\right) e_{1}\right) \mathrm{d} h \\
& =\frac{1}{\kappa_{1}^{l_{1}} \kappa_{2}^{l_{2}}} \frac{1}{\mathrm{i}\left(c_{1} \kappa_{1}-c_{2} \kappa_{2}\right)} \int_{\mathbb{R}^{d}} c_{4}\left(h, x,-\left(c_{1} \kappa_{1}+c_{2} \kappa_{2}\right) e_{1}\right) \mathrm{d} e^{\mathrm{i}\left(c_{1} \kappa_{1}-c_{2} \kappa_{2}\right) h_{1}} \cdots \mathrm{d} h_{d} \\
& =-\frac{1}{\kappa_{1}^{l_{1}} \kappa_{2}^{l_{2}}} \frac{1}{\mathrm{i}\left(c_{1} \kappa_{1}-c_{2} \kappa_{2}\right)} \int_{\mathbb{R}^{d}} e^{\mathrm{i}\left(c_{1} \kappa_{1}-c_{2} \kappa_{2}\right) h_{1}} \partial_{h_{1}} c_{4}\left(h, x,-\left(c_{1} \kappa_{1}+c_{2} \kappa_{2}\right) e_{1}\right) \mathrm{d} h \\
& =(-1)^{n} \frac{1}{\kappa_{1}^{l_{1}} \kappa_{2}^{l_{2}}} \frac{1}{\left.\mathrm{i}\left(c_{1} \kappa_{1}-c_{2} \kappa_{2}\right)\right)^{n}} \int_{\mathbb{R}^{d}} e^{\mathrm{i}\left(c_{1} \kappa_{1}-c_{2} \kappa_{2}\right) h_{1}} \partial_{h_{1}}^{n} c_{4}\left(h, x,-\left(c_{1} \kappa_{1}+c_{2} \kappa_{2}\right) e_{1}\right) \mathrm{d} h,
\end{aligned}
$$

where we use the integrations by parts $n$ times and the fact that $c_{4}(h, x, \xi)$ is $C^{\infty}$ smooth and compactly supported in the $(g, h, x)$ variables. Since $c_{4}(h, x, \xi) \in S_{1,0}^{-m}\left(\mathbb{R}^{2 d} \times \mathbb{R}^{d}\right)$, we have $\left|\partial_{h_{1}}^{n} c_{4}(h, x, \xi)\right| \leq$ $c_{n}(1+|\xi|)^{-m}$ for all positive integer $n$, where $c_{n}$ is independent of $(h, x) \in \mathbb{R}^{2 d}$. Therefore

$$
\begin{aligned}
\left|I\left(x, \kappa_{1}, \kappa_{2}\right)\right| & \lesssim \frac{1}{\kappa_{1}^{l_{1}} \kappa_{2}^{l_{2}}} \frac{1}{\left(1+\left|c_{1} \kappa_{1}-c_{2} \kappa_{2}\right|\right)^{n}} \frac{1}{\left(1+\left|c_{1} \kappa_{1}+c_{2} \kappa_{2}\right|\right)^{m}} \\
& \lesssim \frac{1}{\left(\kappa_{1} \kappa_{2}\right)^{\min \left(l_{1}, l_{2}\right)}} \frac{1}{\left(1+\left|c_{1} \kappa_{1}-c_{2} \kappa_{2}\right|\right)^{n}} \frac{1}{\left(c_{1} \kappa_{1}+c_{2} \kappa_{2}\right)^{m}},
\end{aligned}
$$

where we use the fact that $\kappa_{1} \geq 1, \kappa_{2} \geq 1$. We need to consider the cases where $\left|c_{1} \kappa_{1}-c_{2} \kappa_{2}\right| \geq$ $\left(c_{1} \kappa_{1}+c_{2} \kappa_{2}\right) / 2$ and $\left|c_{1} \kappa_{1}-c_{2} \kappa_{2}\right| \leq\left(c_{1} \kappa_{1}+c_{2} \kappa_{2}\right) / 2$. If $\left|c_{1} \kappa_{1}-c_{2} \kappa_{2}\right| \leq\left(c_{1} \kappa_{1}+c_{2} \kappa_{2}\right) / 2$, a simple calculation shows that $\kappa_{1} \kappa_{2} \geq 3\left(c_{1} \kappa_{1}+c_{2} \kappa_{2}\right)^{2} /\left(16 c_{1} c_{2}\right)$ which implies

$$
\left|I\left(x, \kappa_{1}, \kappa_{2}\right)\right| \lesssim \frac{1}{\left(1+\left|c_{1} \kappa_{1}-c_{2} \kappa_{2}\right|\right)^{n}} \frac{1}{\left(c_{1} \kappa_{1}+c_{2} \kappa_{2}\right)^{m+2 \min \left(l_{1}, l_{2}\right)}} .
$$

If $\left|c_{1} \kappa_{1}-c_{2} \kappa_{2}\right| \geq\left(c_{1} \kappa_{1}+c_{2} \kappa_{2}\right) / 2$, we have

$$
\left|I\left(x, \kappa_{1}, \kappa_{2}\right)\right| \lesssim \frac{1}{\left(1+\left|c_{1} \kappa_{1}-c_{2} \kappa_{2}\right|\right)^{n-2 \min \left(l_{1}, l_{2}\right)}} \frac{1}{\left(c_{1} \kappa_{1}+c_{2} \kappa_{2}\right)^{m+2 \min \left(l_{1}, l_{2}\right)}} .
$$


Noting that the positive integer $n$ is arbitrary, we conclude

$$
\begin{aligned}
\left|I\left(x, \kappa_{1}, \kappa_{2}\right)\right| & \lesssim \frac{1}{\left(1+\left|c_{1} \kappa_{1}-c_{2} \kappa_{2}\right|\right)^{n}} \frac{1}{\left(c_{1} \kappa_{1}+c_{2} \kappa_{2}\right)^{m+2 \min \left(l_{1}, l_{2}\right)}} \\
& \lesssim\left(1+\left|\kappa_{1}-\kappa_{2}\right|\right)^{-n}\left(\kappa_{1}+\kappa_{2}\right)^{-\left(m+2 \min \left(l_{1}, l_{2}\right)\right)},
\end{aligned}
$$

where we use the facts $c_{1} \kappa_{1}+c_{2} \kappa_{2} \leq \min \left(c_{1}, c_{2}\right)\left(\kappa_{1}+\kappa_{2}\right)$ and $\left|c_{1} \kappa_{1}-c_{2} \kappa_{2}\right| \leq c\left|\kappa_{1}-\kappa_{2}\right|$ for some constant $c$. So the estimate (3.22) holds.

Similarly, it is easy to see that $\mathbb{E}\left(\tilde{u}\left(x, \kappa_{1}\right) \tilde{u}\left(x, \kappa_{2}\right)\right)$ is a linear combination of the integral

$$
\tilde{I}\left(x, \kappa_{1}, \kappa_{2}\right):=\frac{1}{\kappa_{1}^{l_{1}} \kappa_{2}^{l_{2}}} \int_{\mathbb{R}^{2 d}} e^{\mathrm{i}\left(c_{1} \kappa_{1}|x-y|+c_{2} \kappa_{2}|x-z|\right)} K(x, y, z) \mathbb{E}(q(y) q(z)) \mathrm{d} y \mathrm{~d} z .
$$

Observe that $\tilde{I}$ is analogous to $I$ where we replace $\kappa_{2}$ with $-\kappa_{2}$. Since the proof of (3.33) allows $\kappa_{2}$ to be negative, we may show that

$$
\begin{aligned}
\left|\tilde{I}\left(x, \kappa_{1}, \kappa_{2}\right)\right| & \lesssim \frac{1}{\kappa_{1}^{l_{1}} \kappa_{2}^{l_{2}}} \frac{1}{\left|c_{1} \kappa_{1}+c_{2} \kappa_{2}\right|^{n}} \frac{1}{\left(1+\left|c_{1} \kappa_{1}-c_{2} \kappa_{2}\right|\right)^{m}} \\
& \lesssim\left(\kappa_{1}+\kappa_{2}\right)^{-n}\left(1+\left|\kappa_{1}-\kappa_{2}\right|\right)^{-m},
\end{aligned}
$$

which shows the estimate (3.23) and completes the proof.

Since $\mathbb{E}\left(\tilde{u}\left(x, \kappa_{1}\right) \overline{\tilde{u}\left(x, \kappa_{2}\right)}\right)$ is a linear combination of $I$ which satisfies the estimate (3.22) , thus the following result is a direct consequence of Lemma 3.5.

Lemma 3.6. For $\kappa_{1}, \kappa_{2} \geq 1$, the estimates

$$
\begin{aligned}
& \left|\mathbb{E}\left(\tilde{u}\left(x, \kappa_{1}\right) \overline{\tilde{u}\left(x, \kappa_{2}\right)}\right)\right| \leq c_{n}\left(\kappa_{1}+\kappa_{2}\right)^{-(m+1)}\left(1+\left|\kappa_{1}-\kappa_{2}\right|\right)^{-n}, \\
& \left|\mathbb{E}\left(\tilde{u}\left(x, \kappa_{1}\right) \tilde{u}\left(x, \kappa_{2}\right)\right)\right| \leq c_{n}\left(\kappa_{1}+\kappa_{2}\right)^{-n}\left(1+\left|\kappa_{1}-\kappa_{2}\right|\right)^{-m} .
\end{aligned}
$$

holds uniformly for $x \in U$, where $n \in \mathbb{N}$ is arbitrary and $c_{n}>0$ is a constant depending only on $n$.

To derive the linear relationship between the scattering data and the function in the principle symbol, it is required to compute the order of $\mathbb{E}\left(|\tilde{u}(x, \kappa)|^{2}\right)$ in terms of $\kappa$. To this end, we study the asymptotic of $I(x, \kappa, \kappa)$ for large $\kappa$.

Lemma 3.7. For $\kappa_{1}=\kappa_{2}=\kappa$, the following asymptotic holds

$$
I(x, \kappa, \kappa)=R_{d}(x, \kappa) \kappa^{-\left(l_{1}+l_{2}+m\right)}+O\left(\kappa^{-\left(l_{1}+l_{2}+m+1\right)}\right),
$$

where $R_{d}(x, \kappa)$ is given by

$$
R_{d}(x, \kappa)=C_{d} \int_{\mathbb{R}^{d}} e^{\mathrm{i}\left(c_{1}-c_{2}\right)|x-y| \kappa} \frac{\left(x_{1}-y_{1}\right)^{m_{1}+n_{1}} \cdots\left(x_{d}-y_{d}\right)^{m_{d}+n_{d}}}{|x-y|^{p_{1}+p_{2}}} \phi(y) \mathrm{d} y
$$

with

$$
C_{2}=-\frac{1}{64} c_{m}, \quad C_{3}=\frac{1}{8} c_{m}, \quad c_{m}=\left(\frac{2}{c_{1}+c_{2}}\right)^{m} .
$$

Proof. Setting $\kappa_{1}=\kappa_{2}=\kappa$ in (3.32) gives

$$
I(x, \kappa, \kappa)=\frac{1}{\kappa^{l_{1}+l_{2}}} \int_{\mathbb{R}^{d}} e^{\mathrm{i}\left(c_{1}-c_{2}\right) \kappa h \cdot e_{1}} c_{4}\left(h, x,-\left(c_{1}+c_{2}\right) \kappa e_{1}\right) \mathrm{d} g \mathrm{~d} h .
$$

The symbol $c_{4}(h, x, \xi) \in S_{1,0}^{-m}\left(\mathbb{R}^{2 d} \times \mathbb{R}^{d}\right)$ can be decomposed into

$$
c_{4}(h, x, \xi)=c_{4}^{p}(h, x, \xi)+r(h, x, \xi),
$$


where $c_{4}^{p}(h, x, \xi) \in S_{1,0}^{-m}\left(\mathbb{R}^{2 d} \times \mathbb{R}^{d}\right)$ is the principal symbol which is given by (3.30) and $r(h, x, \xi) \in$ $S_{1,0}^{-m-1}\left(\mathbb{R}^{2 d} \times \mathbb{R}^{d}\right)$ is the lower order remainder terms which is smooth and compactly supported in $(g, h, x)$-variables. Thus, we have

$$
\begin{aligned}
I(x, \kappa, \kappa) & =\frac{1}{\kappa^{l_{1}+l_{2}}} \int_{\mathbb{R}^{d}} e^{\mathrm{i}\left(c_{1}-c_{2}\right) \kappa h \cdot e_{1}}\left(c_{4}^{p}\left(h, x,-\left(c_{1}+c_{2}\right) \kappa e_{1}\right)+r\left(h, x,-\left(c_{1}+c_{2}\right) \kappa e_{1}\right)\right) \mathrm{d} h \\
& =\frac{1}{\kappa^{l_{1}+l_{2}}} \int_{\mathbb{R}^{d}} e^{\mathrm{i}\left(c_{1}-c_{2}\right) \kappa h \cdot e_{1}} c_{4}^{p}\left(h, x,-\left(c_{1}+c_{2}\right) \kappa e_{1}\right) \mathrm{d} h+O\left(\kappa^{-\left(l_{1}+l_{2}+m+1\right)}\right) .
\end{aligned}
$$

By (3.30),

$$
\begin{aligned}
c_{4}^{p}\left(h, x,-\left(c_{1}+c_{2}\right) \kappa e_{1}\right)= & \phi(y(g, h, x))\left(\left(c_{1}+c_{2}\right)\left|\left(\left(\rho_{11}^{\prime}(g, h, x)\right)^{-1}\right)^{\top} e_{1}\right| \kappa\right)^{-m} \theta(x) \\
& \times\left.\left|\operatorname{det} \rho_{11}^{\prime}(g, h, x)\right|^{-1} H(g, h, x)\right|_{g=0} .
\end{aligned}
$$

Letting $a=\left(c_{1}+c_{2}\right)^{2}\left|\left(\left(\rho_{11}^{\prime}(g, h, x)\right)^{-1}\right)^{\top} e_{1}\right|^{2} \neq 0$, we substitute (3.35) into formula (3.34) and obtain

$$
I(x, \kappa, \kappa)=R_{d}(x, \kappa) \kappa^{-\left(l_{1}+l_{2}+m\right)}+O\left(\kappa^{-\left(l_{1}+l_{2}+m+1\right)}\right),
$$

where

$$
R_{d}(x, \kappa)=\theta(x) \int_{\mathbb{R}^{d}} e^{\mathrm{i}\left(c_{1}-c_{2}\right) \kappa h \cdot e_{1}} \frac{\phi(y(0, h, x)) H(0, h, x)}{a^{\frac{m}{2}}\left|\operatorname{det} \rho_{11}^{\prime}(0, h, x)\right|} \mathrm{d} h .
$$

Next we need to compute $a$. Noting that $a=\left(c_{1}+c_{2}\right)^{2}\left|\left(\left(\rho_{11}^{\prime}(g, h, x)\right)^{-1}\right)^{\top} e_{1}\right|^{2}$, we compute $\left.\rho_{11}^{\prime}(g, h, x)\right)^{-1}$ first.

In two dimensions, we have from the definition of $\rho_{1}$ that

$$
\rho_{11}^{\prime}(g, h, x)=\partial_{g} v=\left[\begin{array}{ll}
\partial_{g_{1}} v_{1} & \partial_{g_{2}} v_{1} \\
\partial_{g_{1}} v_{2} & \partial_{g_{2}} v_{2}
\end{array}\right] .
$$

It is convenient to compute

$$
\rho_{11}^{\prime}(g, h, x)^{-1}=\partial_{v} g=\left[\begin{array}{cc}
\partial_{v_{1}} g_{1} & \partial_{v_{2}} g_{1} \\
\partial_{v_{1}} g_{2} & \partial_{v_{2}} g_{2}
\end{array}\right] .
$$

By the definition of $\eta$, we have $v=y-z, w=y+z$ which implies that $y=(v+w) / 2, z=(w-v) / 2$. Thus we obtain $y_{1}=\left(v_{1}+w_{1}\right) / 2, y_{2}=\left(v_{2}+w_{2}\right) / 2, z_{1}=\left(w_{1}-v_{1}\right) / 2$, and $z_{2}=\left(w_{2}-v_{2}\right) / 2$. When $g=0$ which means $y=z$, we have

$$
\begin{aligned}
& \frac{\partial g_{1}}{\partial v_{1}}=\frac{\partial t_{1}}{\partial v_{1}}-\frac{\partial t_{2}}{\partial v_{1}}=\frac{\partial t_{1}}{\partial y_{1}} \frac{\partial y_{1}}{\partial v_{1}}-\frac{\partial t_{2}}{\partial z_{1}} \frac{\partial z_{1}}{\partial v_{1}}=\frac{1}{4}\left(\frac{y_{1}-x_{1}}{|x-y|}+\frac{z_{1}-x_{1}}{|x-z|}\right)=\frac{1}{2} \frac{y_{1}-x_{1}}{|x-y|}=\frac{1}{2} \sin \alpha, \\
& \frac{\partial g_{1}}{\partial v_{2}}=\frac{\partial t_{1}}{\partial v_{2}}-\frac{\partial t_{2}}{\partial v_{2}}=\frac{\partial t_{1}}{\partial y_{2}} \frac{\partial y_{2}}{\partial v_{2}}-\frac{\partial t_{2}}{\partial z_{2}} \frac{\partial z_{2}}{\partial v_{2}}=\frac{1}{4}\left(\frac{y_{2}-x_{2}}{|x-y|}+\frac{z_{2}-x_{2}}{|x-z|}\right)=\frac{1}{2} \frac{y_{2}-x_{2}}{|x-y|}=\frac{1}{2} \cos \alpha, \\
& \frac{\partial g_{2}}{\partial v_{1}}=\frac{\partial s_{1}}{\partial v_{1}}-\frac{\partial s_{2}}{\partial v_{1}}=\frac{\partial s_{1}}{\partial y_{1}} \frac{\partial y_{1}}{\partial v_{1}}-\frac{\partial s_{2}}{\partial z_{1}} \frac{\partial z_{1}}{\partial v_{1}}=\frac{1}{2}\left(\frac{\partial s_{1}}{\partial y_{1}}+\frac{\partial s_{2}}{\partial z_{1}}\right) \\
& =\frac{1}{2}\left[\frac{y_{1}-x_{1}}{|x-y|} \arcsin \left(\frac{y_{1}-x_{1}}{|x-y|}\right)+\left(1-\left(\frac{y_{1}-x_{1}}{|x-y|}\right)^{2}\right)^{\frac{1}{2}}\right]=\frac{1}{2}(\alpha \sin \alpha+\cos \alpha), \\
& \frac{\partial g_{2}}{\partial v_{2}}=\frac{\partial s_{1}}{\partial v_{2}}-\frac{\partial s_{2}}{\partial v_{2}}=\frac{\partial s_{1}}{\partial y_{2}} \frac{\partial y_{2}}{\partial v_{2}}-\frac{\partial s_{2}}{\partial z_{2}} \frac{\partial z_{2}}{\partial v_{2}}=\frac{1}{2}\left(\frac{\partial s_{1}}{\partial y_{2}}+\frac{\partial s_{2}}{\partial z_{2}}\right) \\
& =\frac{1}{2}\left[\frac{y_{2}-x_{2}}{|x-y|} \arcsin \left(\frac{y_{1}-x_{1}}{|x-y|}\right)-\left(1-\left(\frac{y_{1}-x_{1}}{|x-y|}\right)^{2}\right)^{-\frac{1}{2}} \frac{\left(y_{1}-x_{1}\right)\left(y_{2}-x_{2}\right)}{|x-y|^{2}}\right] \\
& =\frac{1}{2}(\alpha \cos \alpha-\sin \alpha) \text {, }
\end{aligned}
$$


where $\alpha=\arcsin \left(\frac{y_{1}-x_{1}}{|x-y|}\right)$. Hence,

$$
\begin{aligned}
\left(\rho_{11}^{\prime}(0, h, x)\right)^{-1} & =\frac{1}{2}\left[\begin{array}{cc}
\sin \alpha & \cos \alpha \\
\alpha \sin \alpha+\cos \alpha & \alpha \cos \alpha-\sin \alpha
\end{array}\right], \\
\left(\left(\rho_{11}^{\prime}(0, h, x)\right)^{-1}\right)^{T} e_{1} & =\frac{1}{2}(\sin \alpha, \cos \alpha)^{\top} .
\end{aligned}
$$

Thus we obtain $a=\left(c_{1}+c_{2}\right)^{2} / 4$ and $\left|\operatorname{det} \rho_{11}^{\prime}(0, h, x)\right|^{-1}=1 / 4$. Next we focus on the computation of $H(0, h, x)$. From (3.28) we have $H(g, h, x)=K(x, y, z) \operatorname{det}\left(\left(\tau^{-1}\right)^{\prime}(g, h, x)\right)$, thus we compute $\left|\operatorname{det} \tau^{\prime}(0, h, x)\right|$ first. Recalling that $\tau: \mathbb{R}^{6} \rightarrow \mathbb{R}^{6}$ is given by $\tau(g, h, x)=(y, z, x)$, we have

$$
\tau^{\prime}(g, h, x)=\left[\begin{array}{lll}
\partial_{g} y & \partial_{h} y & \partial_{x} y \\
\partial_{g} z & \partial_{h} z & \partial_{x} z \\
\partial_{g} x & \partial_{h} x & \partial_{x} x
\end{array}\right], \quad\left(\tau^{\prime}(g, h, x)\right)^{-1}=\left[\begin{array}{ccc}
\partial_{y} g & \partial_{z} g & \partial_{x} g \\
\partial_{y} h & \partial_{z} h & \partial_{x} h \\
\partial_{y} x & \partial_{z} x & \partial_{x} x
\end{array}\right]
$$

Now we calculate $\partial_{y} g$. Noting $g=\left(t_{1}-t_{2}, s_{1}-s_{2}\right)$, we obtain

$$
\begin{aligned}
\frac{\partial g_{1}}{\partial y_{1}}=\frac{\partial t_{1}}{\partial y_{1}} & =\frac{1}{2} \frac{y_{1}-x_{1}}{|x-y|}=\frac{1}{2} \sin \alpha, \quad \frac{\partial g_{1}}{\partial y_{2}}=\frac{\partial t_{1}}{\partial y_{2}}=\frac{1}{2} \frac{y_{2}-x_{2}}{|x-y|}=\frac{1}{2} \cos \alpha, \\
\frac{\partial g_{2}}{\partial y_{1}}=\frac{\partial s_{1}}{\partial y_{1}} & =\frac{1}{2}\left[\frac{y_{1}-x_{1}}{|x-y|} \arcsin \left(\frac{y_{1}-x_{1}}{|x-y|}\right)+\left(1-\left(\frac{y_{1}-x_{1}}{|x-y|}\right)^{2}\right)^{\frac{1}{2}}\right]=\frac{1}{2}(\alpha \sin \alpha+\cos \alpha), \\
\frac{\partial g_{2}}{\partial y_{2}}=\frac{\partial s_{1}}{\partial y_{2}} & =\frac{1}{2}\left[\frac{y_{2}-x_{2}}{|x-y|} \arcsin \left(\frac{y_{1}-x_{1}}{|x-y|}\right)-\left(1-\left(\frac{y_{1}-x_{1}}{|x-y|}\right)^{2}\right)^{-\frac{1}{2}} \frac{\left(y_{1}-x_{1}\right)\left(y_{2}-x_{2}\right)}{|x-y|^{2}}\right] \\
& =\frac{1}{2}(\alpha \cos \alpha-\sin \alpha) .
\end{aligned}
$$

Thus

$$
\partial_{y} g=\frac{1}{2}\left[\begin{array}{cc}
\sin \alpha & \cos \alpha \\
\alpha \sin \alpha+\cos \alpha & \alpha \cos \alpha-\sin \alpha
\end{array}\right] .
$$

When $g=0$, we have $y=z$. A similar calculation yields that $\partial_{z} g=-\partial_{y} g, \partial_{y} h=\partial_{y} g, \partial_{z} h=\partial_{y} g$. Obviously, $\partial_{y} x=0, \partial_{z} x=0, \partial_{x} x=I$. Hence

$$
\begin{aligned}
& \operatorname{det}\left(\left(\tau^{\prime}(0, h, x)\right)^{-1}\right) \\
& =\frac{1}{2^{4}}\left|\begin{array}{cccc}
\sin \alpha & \cos \alpha & -\sin \alpha & -\cos \alpha \\
\alpha \sin \alpha+\cos \alpha & \alpha \cos \alpha-\sin \alpha & -\alpha \sin \alpha-\cos \alpha & -\alpha \cos \alpha+\sin \alpha \\
\sin \alpha & \cos \alpha & \sin \alpha & \cos \alpha \\
\alpha \sin \alpha+\cos \alpha & \alpha \cos \alpha-\sin \alpha & \alpha \sin \alpha+\cos \alpha & \alpha \cos \alpha-\sin \alpha
\end{array}\right| .
\end{aligned}
$$

A direct calculation shows that $\operatorname{det}\left(\left(\tau^{\prime}(0, h, x)\right)^{-1}\right)=1 / 4$ which $\operatorname{implies} \operatorname{det}\left(\left(\tau^{\prime}(0, h, x)\right)\right)=4$. Substituting these results into (3.36) gives

$$
R_{2}(x, \kappa)=\frac{1}{16} c_{m} \int_{\mathbb{R}^{2}} e^{\mathrm{i}\left(c_{1}-c_{2}\right)|x-y| \kappa} \frac{\left(x_{1}-y_{1}\right)^{m_{1}+n_{1}}\left(x_{2}-y_{2}\right)^{m_{2}+n_{2}}}{|x-y|^{p_{1}+p_{2}}} \phi(y) \mathrm{d} h,
$$

where $(y, z, x)=\tau(g, h, x)$. Noting $\operatorname{det}\left(\frac{\partial h}{\partial y}\right)=-\frac{1}{4}$, we have

$$
R_{2}(x, \kappa)=C_{2} \int_{\mathbb{R}^{2}} e^{\mathrm{i}\left(c_{1}-c_{2}\right)|x-y| \kappa} \frac{\left(x_{1}-y_{1}\right)^{m_{1}+n_{1}}\left(x_{2}-y_{2}\right)^{m_{2}+n_{2}}}{|x-y|^{p_{1}+p_{2}}} \phi(y) \mathrm{d} y .
$$

In three dimensions, by the definition of $\rho_{1}$, we have

$$
\rho_{11}^{\prime}(g, h, x)=\partial_{g} v=\left[\begin{array}{lll}
\partial_{g_{1}} v_{1} & \partial_{g_{2}} v_{1} & \partial_{g_{3}} v_{1} \\
\partial_{g_{1}} v_{2} & \partial_{g_{2}} v_{2} & \partial_{g_{3}} v_{2} \\
\partial_{g_{1}} v_{3} & \partial_{g_{2}} v_{3} & \partial_{g_{3}} v_{3}
\end{array}\right]
$$


which is available after we compute its inverse matrix

$$
\rho_{11}^{\prime}(g, h, x)^{-1}=\partial_{v} g=\left[\begin{array}{lll}
\partial_{v_{1}} g_{1} & \partial_{v_{2}} g_{1} & \partial_{v_{3}} g_{1} \\
\partial_{v_{1}} g_{2} & \partial_{v_{2}} g_{2} & \partial_{v_{3}} g_{2} \\
\partial_{v_{1}} g_{3} & \partial_{v_{2}} g_{3} & \partial_{v_{3}} g_{3}
\end{array}\right]
$$

By the definition of $\eta$, we have $v=y-z, w=y+z$ which implies that $y=(v+w) / 2, z=(w-v) / 2$. Thus $y_{1}=\left(v_{1}+w_{1}\right) / 2, y_{2}=\left(v_{2}+w_{2}\right) / 2, y_{3}=\left(v_{3}+w_{3}\right) / 2, z_{1}=\left(w_{1}-v_{1}\right) / 2, z_{2}=\left(w_{2}-v_{2}\right) / 2$, and $z_{3}=\left(w_{3}-v_{3}\right) / 2$. Define

$$
\alpha=\arccos \left(\frac{y_{3}-x_{3}}{|x-y|}\right), \quad \beta=\arctan \left(\frac{y_{2}-x_{2}}{y_{1}-x_{1}}\right) .
$$

A direct computation shows that

$$
\frac{y_{1}-x_{1}}{|x-y|}=\sin \alpha \cos \beta, \quad \frac{y_{2}-x_{2}}{|x-y|}=\sin \alpha \sin \beta, \quad \frac{y_{3}-x_{3}}{|x-y|}=\cos \alpha .
$$

When $g=0$ which implies $y=z$, we have

$$
\begin{aligned}
& \frac{\partial g_{1}}{\partial v_{1}}=\frac{\partial t_{1}}{\partial v_{1}}-\frac{\partial t_{2}}{\partial v_{1}}=\frac{\partial t_{1}}{\partial y_{1}} \frac{\partial y_{1}}{\partial v_{1}}-\frac{\partial t_{2}}{\partial z_{1}} \frac{\partial z_{1}}{\partial v_{1}}=\frac{1}{4}\left(\frac{y_{1}-x_{1}}{|x-y|}+\frac{z_{1}-x_{1}}{|x-z|}\right)=\frac{1}{2} \frac{y_{1}-x_{1}}{|x-y|}=\frac{1}{2} \sin \alpha \cos \beta, \\
& \frac{\partial g_{1}}{\partial v_{2}}=\frac{\partial t_{1}}{\partial v_{2}}-\frac{\partial t_{2}}{\partial v_{2}}=\frac{\partial t_{1}}{\partial y_{2}} \frac{\partial y_{2}}{\partial v_{2}}-\frac{\partial t_{2}}{\partial z_{2}} \frac{\partial z_{2}}{\partial v_{2}}=\frac{1}{4}\left(\frac{y_{2}-x_{2}}{|x-y|}+\frac{z_{2}-x_{2}}{|x-z|}\right)=\frac{1}{2} \frac{y_{2}-x_{2}}{|x-y|}=\frac{1}{2} \sin \alpha \sin \beta, \\
& \frac{\partial g_{1}}{\partial v_{3}}=\frac{\partial r_{1}}{\partial v_{3}}-\frac{\partial r_{2}}{\partial v_{3}}=\frac{\partial r_{1}}{\partial y_{3}} \frac{\partial y_{3}}{\partial v_{3}}-\frac{\partial r_{2}}{\partial z_{3}} \frac{\partial z_{3}}{\partial v_{3}}=\frac{1}{4}\left(\frac{y_{3}-x_{3}}{|x-y|}+\frac{z_{3}-x_{3}}{|x-z|}\right)=\frac{1}{2} \frac{y_{3}-x_{3}}{|x-y|}=\frac{1}{2} \cos \alpha, \\
& \frac{\partial g_{2}}{\partial v_{1}}=\frac{\partial s_{1}}{\partial v_{1}}-\frac{\partial s_{2}}{\partial v_{1}}=\frac{\partial s_{1}}{\partial y_{1}} \frac{\partial y_{1}}{\partial v_{1}}-\frac{\partial s_{2}}{\partial z_{1}} \frac{\partial z_{1}}{\partial v_{1}}=\frac{1}{2}\left(\frac{\partial s_{1}}{\partial y_{1}}+\frac{\partial s_{2}}{\partial z_{1}}\right) \\
& =\frac{1}{2}\left[\frac{y_{1}-x_{1}}{|x-y|} \arccos \left(\frac{y_{3}-x_{3}}{|x-y|}\right)+\left(1-\left(\frac{y_{3}-x_{3}}{|x-y|}\right)^{2}\right)^{-\frac{1}{2}} \frac{\left(y_{3}-x_{3}\right)\left(y_{1}-x_{1}\right)}{|x-y|^{2}}\right] \\
& =\frac{1}{2} \cos \beta(\alpha \sin \alpha+\cos \alpha) \text {, } \\
& \frac{\partial g_{2}}{\partial v_{2}}=\frac{\partial s_{1}}{\partial v_{2}}-\frac{\partial s_{2}}{\partial v_{2}}=\frac{\partial s_{1}}{\partial y_{2}} \frac{\partial y_{2}}{\partial v_{2}}-\frac{\partial s_{2}}{\partial z_{2}} \frac{\partial z_{2}}{\partial v_{2}}=\frac{1}{2}\left(\frac{\partial s_{1}}{\partial y_{2}}+\frac{\partial s_{2}}{\partial z_{2}}\right) \\
& =\frac{1}{2}\left[\frac{y_{2}-x_{2}}{|x-y|} \arccos \left(\frac{y_{3}-x_{3}}{|x-y|}\right)+\left(1-\left(\frac{y_{3}-x_{3}}{|x-y|}\right)^{2}\right)^{-\frac{1}{2}} \frac{\left(y_{3}-x_{3}\right)\left(y_{2}-x_{2}\right)}{|x-y|^{2}}\right] \\
& =\frac{1}{2} \sin \beta(\alpha \sin \alpha+\cos \alpha) \text {, } \\
& \frac{\partial g_{2}}{\partial v_{3}}=\frac{\partial s_{1}}{\partial v_{3}}-\frac{\partial s_{2}}{\partial v_{3}}=\frac{\partial s_{1}}{\partial y_{3}} \frac{\partial y_{3}}{\partial v_{3}}-\frac{\partial s_{2}}{\partial z_{3}} \frac{\partial z_{3}}{\partial v_{3}}=\frac{1}{2}\left(\frac{\partial s_{1}}{\partial y_{3}}+\frac{\partial s_{2}}{\partial z_{3}}\right) \\
& =\frac{1}{2}\left[\frac{y_{3}-x_{3}}{|x-y|} \arccos \left(\frac{y_{3}-x_{3}}{|x-y|}\right)+\left(1-\left(\frac{y_{3}-x_{3}}{|x-y|}\right)^{2}\right)^{\frac{1}{2}}\right]=\frac{1}{2}(\alpha \cos \alpha-\sin \alpha) \text {, }
\end{aligned}
$$




$$
\begin{aligned}
\frac{\partial g_{3}}{\partial v_{1}} & =\frac{\partial r_{1}}{\partial v_{1}}-\frac{\partial r_{2}}{\partial v_{1}}=\frac{\partial r_{1}}{\partial y_{1}} \frac{\partial y_{1}}{\partial v_{1}}-\frac{\partial r_{2}}{\partial z_{1}} \frac{\partial z_{1}}{\partial v_{1}}=\frac{1}{2}\left(\frac{\partial r_{1}}{\partial y_{1}}+\frac{\partial r_{2}}{\partial z_{1}}\right) \\
& =\frac{1}{2}\left[\frac{y_{1}-x_{1}}{|x-y|} \arctan \left(\frac{y_{2}-x_{2}}{y_{1}-x_{1}}\right)-\frac{|x-y|}{y_{1}-x_{1}} \frac{y_{2}-x_{2}}{\sqrt{\left(y_{1}-x_{1}\right)^{2}+\left(y_{2}-x_{2}\right)^{2}}}\right] \\
& =\frac{1}{2}\left(\beta \sin \alpha \cos \beta-\frac{\sin \beta}{\sin \alpha \cos \beta}\right) \\
\frac{\partial g_{3}}{\partial v_{2}} & =\frac{\partial r_{1}}{\partial v_{2}}-\frac{\partial r_{2}}{\partial v_{2}}=\frac{\partial r_{1}}{\partial y_{2}} \frac{\partial y_{2}}{\partial v_{2}}-\frac{\partial r_{2}}{\partial z_{2}} \frac{\partial z_{2}}{\partial v_{2}}=\frac{1}{2}\left(\frac{\partial r_{1}}{\partial y_{2}}+\frac{\partial r_{2}}{\partial z_{2}}\right) \\
& =\frac{1}{2}\left[\frac{y_{2}-x_{2}}{|x-y|} \arctan \left(\frac{y_{2}-x_{2}}{y_{1}-x_{1}}\right)+\frac{1}{\sqrt{\left(y_{1}-x_{1}\right)^{2}+\left(y_{2}-x_{2}\right)^{2}}}\right] \\
& =\frac{1}{2}\left(\beta \sin \alpha \sin \beta+\frac{1}{\sin \alpha}\right), \\
\frac{\partial g_{3}}{\partial v_{3}} & =\frac{\partial r_{1}}{\partial v_{3}}-\frac{\partial r_{2}}{\partial v_{3}}=\frac{\partial r_{1}}{\partial y_{3}} \frac{\partial y_{3}}{\partial v_{3}}-\frac{\partial r_{2}}{\partial z_{3}} \frac{\partial z_{3}}{\partial v_{3}}=\frac{1}{2}\left(\frac{\partial r_{1}}{\partial y_{3}}+\frac{\partial r_{2}}{\partial z_{3}}\right) \\
& =\frac{1}{2}\left[\frac{y_{3}-x_{3}}{|x-y|} \arctan \left(\frac{y_{2}-x_{2}}{y_{1}-x_{1}}\right)\right]=\frac{1}{2} \beta \cos \alpha .
\end{aligned}
$$

Hence,

$$
\left(\rho_{11}^{\prime}(0, h, x)\right)^{-1}=\frac{1}{2}\left[\begin{array}{ccc}
\sin \alpha \cos \beta & \sin \alpha \sin \beta & \cos \alpha \\
\cos \beta(\alpha \sin \alpha+\cos \alpha) & \sin \beta(\alpha \sin \alpha+\cos \alpha) & \alpha \cos \alpha-\sin \alpha \\
\beta \sin \alpha \cos \beta-\frac{\sin \beta}{\sin \alpha \cos \beta} & \beta \sin \alpha \sin \beta+\frac{1}{\sin \alpha} & \beta \cos \alpha
\end{array}\right],
$$

which gives

$$
\left(\left(\rho_{11}^{\prime}(0, h, x)\right)^{-1}\right)^{\top} e_{1}=\frac{1}{2}(\sin \alpha \cos \beta, \sin \alpha \sin \beta, \cos \alpha)^{\top} .
$$

Thus we obtain $a=\left(c_{1}+c_{2}\right)^{2} / 4$ and $\left|\operatorname{det} \rho_{11}^{\prime}(0, h, x)\right|^{-1}=\frac{1}{8 \sin \alpha \cos \beta}=\frac{1}{8} \frac{|y-x|}{y_{1}-x_{1}}$. Next we focus on the computation of $H(0, h, x)$, which requires to compute $\left|\operatorname{det} \tau^{\prime}(0, h, x)\right|$ first. Recalling that $\tau^{-1}: \mathbb{R}^{9} \rightarrow \mathbb{R}^{9}$ is given by $\tau^{-1}(g, h, x)=(y, z, x)$, we have

$$
\left(\tau^{-1}\right)^{\prime}(g, h, x)=\left[\begin{array}{lll}
\partial_{g} y & \partial_{h} y & \partial_{x} y \\
\partial_{g} z & \partial_{h} z & \partial_{x} z \\
\partial_{g} x & \partial_{h} x & \partial_{x} x
\end{array}\right], \quad\left(\left(\tau^{-1}\right)^{\prime}(g, h, x)\right)^{-1}=\left[\begin{array}{ccc}
\partial_{y} g & \partial_{z} g & \partial_{x} g \\
\partial_{y} h & \partial_{z} h & \partial_{x} h \\
\partial_{y} x & \partial_{z} x & \partial_{x} x
\end{array}\right] .
$$

Now we calculate $\frac{\partial g}{\partial y}$. Noting $g=\left(t_{1}-t_{2}, s_{1}-s_{2}, r_{1}-r_{2}\right)$, we obtain that

$$
\frac{\partial g}{\partial y}=\frac{1}{2}\left[\begin{array}{ccc}
\sin \alpha \cos \beta & \sin \alpha \sin \beta & \cos \alpha \\
\cos \beta(\alpha \sin \alpha+\cos \alpha) & \sin \beta(\alpha \sin \alpha+\cos \alpha) & \alpha \cos \alpha-\sin \alpha \\
\beta \sin \alpha \cos \beta-\frac{\sin \beta}{\sin \alpha \cos \beta} & \beta \sin \alpha \sin \beta+\frac{1}{\sin \alpha} & \beta \cos \alpha
\end{array}\right] .
$$

When $g=0$, we have $y=z$. A simple calculation yields that $\frac{\partial g}{\partial z}=-\frac{\partial g}{\partial y}, \frac{\partial h}{\partial y}=\frac{\partial g}{\partial y}, \frac{\partial h}{\partial z}=\frac{\partial g}{\partial y}$. It is clear to note that $\frac{\partial x}{\partial y}=0, \frac{\partial x}{\partial z}=0, \frac{\partial x}{\partial x}=I$. Hence

$$
\operatorname{det}\left(\left(\left(\tau^{-1}\right)^{\prime}(0, h, x)\right)^{-1}\right)=\frac{1}{8} \frac{|y-x|^{2}}{\left(y_{1}-x_{1}\right)^{2}},
$$

which implies

$$
\operatorname{det}\left(\left(\tau^{-1}\right)^{\prime}(0, h, x)\right)=8 \frac{\left(y_{1}-x_{1}\right)^{2}}{|y-x|^{2}} .
$$


Substituting these results into (3.36) gives

$$
\begin{aligned}
R_{3}(x, \kappa)=c_{m} & \int_{\mathbb{R}^{3}} e^{\mathrm{i}\left(c_{1}-c_{2}\right) \kappa h \cdot e_{1}} \frac{\left(x_{1}-y_{1}\right)^{m_{1}+n_{1}}\left(x_{2}-y_{2}\right)^{m_{2}+n_{2}}\left(x_{3}-y_{3}\right)^{m_{3}+n_{3}}}{|x-y|^{p_{1}+p_{2}}} \\
& \times \frac{\left(y_{1}-x_{1}\right)}{|y-x|} \phi(y(0, h, x)) \mathrm{d} h,
\end{aligned}
$$

where $(y, z, x)=\tau^{-1}(g, h, x)$. Noting $\operatorname{det}\left(\frac{\partial h}{\partial y}\right)=\frac{1}{8} \frac{|x-y|}{y_{1}-x_{1}}$, we arrive at

$$
R_{3}(x, \kappa)=C_{3} \int_{\mathbb{R}^{3}} e^{\mathrm{i}\left(c_{1}-c_{2}\right)|x-y| \kappa} \frac{\left(x_{1}-y_{1}\right)^{m_{1}+n_{1}}\left(x_{2}-y_{2}\right)^{m_{2}+n_{2}}\left(x_{3}-y_{3}\right)^{m_{3}+n_{3}}}{|x-y|^{p_{1}+p_{2}}} \phi(y) \mathrm{d} y,
$$

which completes the proof.

Now we are ready to estimate the order of $\mathbb{E}\left(|\tilde{u}(x, \kappa)|^{2}\right)$. Setting $\kappa_{1}=\kappa_{2}=\kappa$ in (3.20) and applying Lemma 3.7, we obtain

$$
\mathbb{E}\left(|\tilde{u}(x, \kappa)|^{2}\right)=T_{\mathrm{A}}^{(2)}(x) \kappa^{-(m+1)}+O\left(\kappa^{-(m+2)}\right),
$$

where

$$
T_{\mathrm{A}}^{(2)}(x)=\frac{C_{2}}{8 \pi} \int_{\mathbb{R}^{2}} \frac{1}{|x-y|} \phi(y) \mathrm{d} y .
$$

Before presenting the main result, we need the following lemma.

Lemma 3.8. Let $V_{1}, V_{2} \subset \mathbb{R}^{d}$ be two open, bounded, and simply connected domains with positive distance. For some positive integer $l$ and $\phi \in C_{0}^{\infty}\left(V_{1}\right)$, define the integral

$$
T(x)=\int_{\mathbb{R}^{d}} \frac{1}{|x-y|^{l}} \phi(y) \mathrm{d} y, \quad x \in V_{2} .
$$

Then $T(x), x \in V_{2}$ uniquely determines the function $\phi$.

Proof. A simple calculation yields

$$
\Delta_{x}|x-y|^{-n}=n^{2}|x-y|^{-n-2}, \quad n \in \mathbb{N},
$$

which implies

$$
\Delta^{n} T(x)=c_{n} \int_{V_{1}} \frac{1}{|x-y|^{l+2 n}} \phi(y) \mathrm{d} y,
$$

where $c_{n}$ is a constant depending on $n$. Since $T(x)$ is known in an open set $V_{2}$ which has a positive distance to the support of $\phi \in C_{0}^{\infty}\left(\mathbb{R}^{2}\right)$, so as $\Delta^{n} T(x), n \in \mathbb{N}$ is known in the set $V_{2}$. A linear combination of $\Delta^{n} T(x)$ shows that the integral

$$
\int_{V_{1}} \frac{1}{|x-y|^{l}} P\left(\frac{1}{|x-y|^{2}}\right) \phi(y) \mathrm{d} y
$$

is known in the set $V_{2}$, where $P(t)=\sum_{j=0}^{J} a_{j} t^{j}$ is a polynomial of order $J \in \mathbb{N}$. In (3.39), by changing the integral variables, we deduce

$$
\begin{aligned}
\int_{V_{1}} \frac{1}{|x-y|^{l}} P\left(\frac{1}{|x-y|^{2}}\right) \phi(y) \mathrm{d} y & =\int_{r_{1}}^{r_{2}} \frac{1}{r^{l}} P\left(\frac{1}{r^{2}}\right) \int_{|y-x|=r} \phi(y) \mathrm{d} s(y) \mathrm{d} r \\
& =\int_{r_{1}}^{r_{2}} \frac{1}{r^{l}} P\left(\frac{1}{r^{2}}\right) S(x, r) \mathrm{d} r \\
& =\frac{1}{2} \int_{\frac{1}{r_{2}^{2}}}^{\frac{1}{r_{1}^{2}}} P(t) S\left(x, \frac{1}{\sqrt{t}}\right) t^{\frac{l-3}{2}} \mathrm{~d} t
\end{aligned}
$$


where $S(x, r)=\int_{|y-x|=r} \phi(y) \mathrm{d} s(y)$ denotes the integral of $\phi(y)$ along the circle $|y-x|=r, r_{1}=$ $\min _{y \in V_{1}}|x-y|$ and $r_{2}=\max _{y \in V_{1}}|x-y|$ denote the minimum and the maximum distance between the fixed point $x \in V_{2}$ and the domain $V_{1}$, respectively. Due to $\phi \in C_{0}^{\infty}\left(\mathbb{R}^{2}\right)$, the function $S(x, r)$ is continuous with respect to $r$ and is compact supported in the interval $\left[r_{1}, r_{2}\right]$. We obtain $S\left(x, \frac{1}{\sqrt{t}}\right) t^{\frac{l-3}{2}}$ is continuous in $\left[r_{2}^{-2}, r_{1}^{-2}\right]$. Note that the polynomial function $P(t)$ is dense in $C\left(\left[r_{2}^{-2}, r_{1}^{-2}\right]\right)$, thus the function $S\left(x, \frac{1}{\sqrt{t}}\right) t^{\frac{l-3}{2}}$ is uniquely determined which implies $S(x, r)$ is uniquely determined for all $r>0$.

Let $g(x)=e^{-\frac{|x|^{2}}{2}}$ for $x \in \mathbb{R}^{2}$, then we have

$$
\begin{aligned}
(g * \phi)(x) & =\int_{\mathbb{R}^{2}} e^{-\frac{|x-y|^{2}}{2}} \phi(y) \mathrm{d} y=\int_{V_{1}} e^{-\frac{|x-y|^{2}}{2}} \phi(y) \mathrm{d} y \\
& =\int_{r_{1}}^{r_{2}} e^{-\frac{r^{2}}{2}} \int_{|y-x|=r} \phi(y) \mathrm{d} y \mathrm{~d} r=\int_{r_{1}}^{r_{2}} e^{-\frac{r^{2}}{2}} S(x, r) \mathrm{d} r .
\end{aligned}
$$

Since $S(x, r)$ is uniquely determined for all $r>0$, we can compute the convolution $g * \phi$ by (3.40) for $x \in V_{2}$. Because $V_{2}$ is open and $g * \phi$ is real analytic, hence $g * \phi$ is known everywhere, and the Fourier transform $\mathcal{F}(g * \phi)$ is known everywhere. Since

$$
\begin{aligned}
\mathcal{F} g(\xi) & =\int_{\mathbb{R}^{2}} e^{-\mathrm{i} x \cdot \xi} g(x) \mathrm{d} x=\int_{\mathbb{R}^{2}} e^{-\left(\frac{|x|^{2}}{2}+\mathrm{i} x \cdot \xi\right)} \mathrm{d} x \\
& =\int_{\mathbb{R}^{2}} e^{-\frac{1}{2}\left(x_{1}^{2}+2 \mathrm{i} x_{1} \xi_{1}\right)} e^{-\frac{1}{2}\left(x_{2}^{2}+2 \mathrm{i} x_{2} \xi_{2}\right)} \mathrm{d} x \\
& =e^{-\frac{1}{2}|\xi|^{2}} \int_{\mathbb{R}} e^{-\frac{1}{2}\left(x_{1}+\mathrm{i} \xi_{1}\right)^{2}} \mathrm{~d} x_{1} \int_{\mathbb{R}} e^{-\frac{1}{2}\left(x_{2}+\mathrm{i} \xi_{2}\right)^{2}} \mathrm{~d} x_{2} \\
& =2 \pi e^{-\frac{1}{2}|\xi|^{2}}
\end{aligned}
$$

we conclude $\mathcal{F} g$ is smooth and non-zero all over $\mathbb{R}^{2}$. Therefore $\mathcal{F} \phi=\mathcal{F}(g * \phi) / \mathcal{F} g$ is uniquely determined which shows that $\phi$ is uniquely determined.

We are in the position to present the main result for the time-harmonic acoustic waves.

Theorem 3.9. Let the external source $f$ be a microlocally isotropic Gaussian random field which satisfies Assumption $B$. Then for all $x \in U$, it holds almost surely that

$$
\lim _{Q \rightarrow \infty} \frac{1}{Q-1} \int_{1}^{Q} \kappa^{m+1}|u(x, \kappa)|^{2} \mathrm{~d} \kappa=T_{\mathrm{A}}^{(2)}(x) .
$$

Moreover, the scattering data $T_{\mathrm{A}}^{(2)}(x), x \in U$ uniquely determines the micro-correlation strength $\phi$ through the linear relation (3.38).

Proof. A simple calculation shows that

$$
\begin{aligned}
& \frac{1}{Q-1} \int_{1}^{Q} \kappa^{m+1}|u(x, \kappa)|^{2} \mathrm{~d} \kappa \\
= & \frac{1}{Q-1} \int_{1}^{Q} \kappa^{m+1}|\tilde{u}(x, \kappa)+u(x, \kappa)-\tilde{u}(x, \kappa)|^{2} \mathrm{~d} \kappa \\
= & \frac{1}{Q-1} \int_{1}^{Q} \kappa^{m+1}|\tilde{u}(x, \kappa)|^{2} \mathrm{~d} \kappa+\frac{1}{Q-1} \int_{1}^{Q} \kappa^{m+1}|u(x, \kappa)-\tilde{u}(x, \kappa)|^{2} \mathrm{~d} \kappa \\
& +\frac{2}{Q-1} \int_{1}^{Q} \kappa^{m+1} \Re[\overline{\tilde{u}(x, \kappa)}(u(x, \kappa)-\tilde{u}(x, \kappa))] \mathrm{d} \kappa .
\end{aligned}
$$


It is clear that (3.41) follows as long as we show that

$$
\begin{aligned}
& \lim _{Q \rightarrow \infty} \frac{1}{Q-1} \int_{1}^{Q} \kappa^{m+1}|\tilde{u}(x, \kappa)|^{2} \mathrm{~d} \kappa=T_{\mathrm{A}}^{(2)}(x), \\
& \lim _{Q \rightarrow \infty} \frac{1}{Q-1} \int_{1}^{Q} \kappa^{m+1}|u(x, \kappa)-\tilde{u}(x, \kappa)|^{2} \mathrm{~d} \kappa=0, \\
& \lim _{Q \rightarrow \infty} \frac{2}{Q-1} \int_{1}^{Q} \kappa^{m+1} \Re[\overline{\tilde{u}(x, \kappa)}(u(x, \kappa)-\tilde{u}(x, \kappa))] \mathrm{d} \kappa=0 .
\end{aligned}
$$

To prove (3.42), we define $Y(x, \kappa)=\kappa^{m+1}\left(|\tilde{u}(x, \kappa)|^{2}-\mathbb{E}|\tilde{u}(x, \kappa)|^{2}\right)$. Since

$$
\int_{1}^{Q} \kappa^{m+1}|\tilde{u}(x, \kappa)|^{2} \mathrm{~d} \kappa=\int_{1}^{Q} \kappa^{m+1} \mathbb{E}|\tilde{u}(x, \kappa)|^{2} \mathrm{~d} \kappa+\int_{1}^{Q} Y(x, \kappa) \mathrm{d} \kappa,
$$

(3.42) holds as long as we prove

$$
\lim _{Q \rightarrow \infty} \frac{1}{Q-1} \int_{1}^{Q} \kappa^{m+1} \mathbb{E}|\tilde{u}(x, \kappa)|^{2} \mathrm{~d} \kappa=T_{\mathrm{A}}^{(2)}(x), \quad \lim _{Q \rightarrow \infty} \frac{1}{Q-1} \int_{1}^{Q} Y(x, \kappa) \mathrm{d} \kappa=0 .
$$

By (3.37), it is easy to see that

$$
\frac{1}{Q-1} \int_{1}^{Q} \kappa^{m+1} \mathbb{E}|\tilde{u}(x, \kappa)|^{2} \mathrm{~d} \kappa=\frac{1}{Q-1} \int_{1}^{Q}\left(T_{A}^{(2)}(x)+O\left(\kappa^{-1}\right)\right) \mathrm{d} \kappa .
$$

Clearly, we have

$$
\frac{1}{Q-1} \int_{1}^{Q} T_{\mathrm{A}}^{(2)}(x) \mathrm{d} \kappa=T_{\mathrm{A}}^{(2)}(x)
$$

and

$$
\left|\frac{1}{Q-1} \int_{1}^{Q} O\left(\kappa^{-1}\right) \mathrm{d} \kappa\right| \lesssim \frac{1}{Q-1} \int_{1}^{Q} \kappa^{-1} \mathrm{~d} \kappa=\frac{\ln Q}{Q-1} \rightarrow 0 \quad \text { as } Q \rightarrow \infty
$$

Hence,

$$
\lim _{Q \rightarrow \infty} \frac{1}{Q-1} \int_{1}^{Q} \kappa^{m+1} \mathbb{E}|\tilde{u}(x, \kappa)|^{2} \mathrm{~d} \kappa=T_{A}^{(2)}(x) .
$$

To prove (3.42), it suffices to show

$$
\lim _{Q \rightarrow \infty} \frac{1}{Q-1} \int_{1}^{Q} Y(x, \kappa) \mathrm{d} \kappa=0 .
$$

By the definition of $Y(x, \kappa)$, we obtain

$$
\begin{aligned}
Y(x, \kappa) & =\kappa^{m+1}\left(|\tilde{u}(x, \kappa)|^{2}-\mathbb{E}|\tilde{u}(x, \kappa)|^{2}\right) \\
& =\kappa^{m+1}\left((\Re \tilde{u}(x, \kappa))^{2}-\mathbb{E}(\Re \tilde{u}(x, \kappa))^{2}+(\Im \tilde{u}(x, \kappa))^{2}-\mathbb{E}(\Im \tilde{u}(x, \kappa))^{2}\right) .
\end{aligned}
$$

Therefore

$$
\mathbb{E}\left(Y\left(x, \kappa_{1}\right) Y\left(x, \kappa_{2}\right)\right)=I_{A, 1}+I_{A, 2}+I_{A, 3}+I_{A, 4},
$$


where

$$
\begin{aligned}
& I_{A, 1}=\kappa_{1}^{m+1} \kappa_{2}^{m+1} \mathbb{E}\left[\left(\left(\Re \tilde{u}\left(x, \kappa_{1}\right)\right)^{2}-\mathbb{E}\left(\Re \tilde{u}\left(x, \kappa_{1}\right)\right)^{2}\right)\left(\left(\Re \tilde{u}\left(x, \kappa_{2}\right)\right)^{2}-\mathbb{E}\left(\Re \tilde{u}\left(x, \kappa_{2}\right)\right)^{2}\right)\right], \\
& I_{A, 2}=\kappa_{1}^{m+1} \kappa_{2}^{m+1} \mathbb{E}\left[\left(\left(\Re \tilde{u}\left(x, \kappa_{1}\right)\right)^{2}-\mathbb{E}\left(\Re \tilde{u}\left(x, \kappa_{1}\right)\right)^{2}\right)\left(\left(\Im \tilde{u}\left(x, \kappa_{2}\right)\right)^{2}-\mathbb{E}\left(\Im \tilde{u}\left(x, \kappa_{2}\right)\right)^{2}\right)\right], \\
& I_{A, 3}=\kappa_{1}^{m+1} \kappa_{2}^{m+1} \mathbb{E}\left[\left(\left(\Im \tilde{u}\left(x, \kappa_{1}\right)\right)^{2}-\mathbb{E}\left(\Im \tilde{u}\left(x, \kappa_{1}\right)\right)^{2}\right)\left(\left(\Re \tilde{u}\left(x, \kappa_{2}\right)\right)^{2}-\mathbb{E}\left(\Re \tilde{u}\left(x, \kappa_{2}\right)\right)^{2}\right)\right], \\
& I_{A, 4}=\kappa_{1}^{m+1} \kappa_{2}^{m+1} \mathbb{E}\left[\left(\left(\Im \tilde{u}\left(x, \kappa_{1}\right)\right)^{2}-\mathbb{E}\left(\Im \tilde{u}\left(x, \kappa_{1}\right)\right)^{2}\right)\left(\left(\Im \tilde{u}\left(x, \kappa_{2}\right)\right)^{2}-\mathbb{E}\left(\Im \tilde{u}\left(x, \kappa_{2}\right)\right)^{2}\right)\right] .
\end{aligned}
$$

Combing the expression of $\tilde{u}(x, \kappa)$ and the assumption $\mathbb{E}(f)=0$ gives that both $\Re \tilde{u}(x, \kappa)$ and $\Im \tilde{u}(x, \kappa)$ are zero-mean Gaussian random variables. Applying Lemmas 2.3 and 3.6 leads to

$$
\begin{aligned}
I_{A, 1} & =2 \kappa_{1}^{m+1} \kappa_{2}^{m+1}\left[\mathbb{E}\left(\Re \tilde{u}\left(x, \kappa_{1}\right) \Re \tilde{u}\left(x, \kappa_{2}\right)\right)\right]^{2} \\
& =\frac{1}{2} \kappa_{1}^{m+1} \kappa_{2}^{m+1}\left[\mathbb{E}\left(\Re\left(\tilde{u}\left(x, \kappa_{1}\right) \tilde{u}\left(x, \kappa_{2}\right)\right)+\Re\left(\tilde{u}\left(x, \kappa_{1}\right) \overline{\tilde{u}\left(x, \kappa_{2}\right)}\right)\right)\right]^{2} \\
& \lesssim\left[\frac{\kappa_{1}^{\frac{m+1}{2}} \kappa_{2}^{\frac{m+1}{2}}}{\left(\kappa_{1}+\kappa_{2}\right)^{n}\left(1+\left|\kappa_{1}-\kappa_{2}\right|\right)^{m}}+\frac{\kappa_{1}^{\frac{m+1}{2}} \kappa_{2}^{\frac{m+1}{2}}}{\left(\kappa_{1}+\kappa_{2}\right)^{m+1}\left(1+\left|\kappa_{1}-\kappa_{2}\right|\right)^{n}}\right]^{2} \\
& \lesssim\left[\frac{1}{\left(1+\left|\kappa_{1}-\kappa_{2}\right|\right)^{m}}+\frac{1}{\left(1+\left|\kappa_{1}-\kappa_{2}\right|\right)^{n}}\right]^{2} .
\end{aligned}
$$

We can obtain the same estimates for $I_{A, 2}, I_{A, 3}$, and $I_{A, 4}$ by the similar arguments. Thus, an application of Lemma 2.4 gives that

$$
\lim _{Q \rightarrow \infty} \frac{1}{Q-1} \int_{1}^{Q} Y(x, \kappa) \mathrm{d} \kappa=0 .
$$

To prove (3.43), we obtain from Lemma 3.4 that

$$
\begin{aligned}
& \left|\frac{1}{Q-1} \int_{1}^{Q} \kappa^{m+1}\right| u(x, \kappa)-\left.\tilde{u}(x, \kappa)\right|^{2} \mathrm{~d} \kappa \mid \lesssim \frac{1}{Q-1} \int_{1}^{Q} \kappa^{m+1} \kappa^{-7} \mathrm{~d} \kappa \\
& =\frac{1}{Q-1} \int_{1}^{Q} \kappa^{m-6} \mathrm{~d} \kappa=\frac{1}{m-5} \frac{Q^{m-5}-1}{Q-1} \rightarrow 0 \quad \text { as } Q \rightarrow \infty .
\end{aligned}
$$

To prove (3.44), by the Hölder inequality, we have

$$
\begin{aligned}
& \left|\frac{2}{Q-1} \int_{1}^{Q} \kappa^{m+1} \Re[\overline{\tilde{u}(x, \kappa)}(u(x, \kappa)-\tilde{u}(x, \kappa))] \mathrm{d} \kappa\right| \\
& \leq \frac{2}{Q-1} \int_{1}^{Q} \kappa^{m+1}|\tilde{u}(x, \kappa)||u(x, \kappa)-\tilde{u}(x, \kappa)| \mathrm{d} \kappa \\
& \leq 2\left[\frac{1}{Q-1} \int_{1}^{Q} \kappa^{m+1}|\tilde{u}(x, \kappa)|^{2} \mathrm{~d} \kappa\right]^{\frac{1}{2}}\left[\frac{1}{Q-1} \int_{1}^{Q} \kappa^{m+1}|u(x, \kappa)-\tilde{u}(x, \kappa)|^{2} \mathrm{~d} \kappa\right]^{\frac{1}{2}} \\
& \rightarrow 2 T_{\mathrm{A}}^{(2)}(x)^{\frac{1}{2}} \cdot 0=0 \quad \text { as } Q \rightarrow \infty .
\end{aligned}
$$

Hence, (3.42) - (3.44) hold which means that (3.41) holds. The unique determination of $\phi$ by the scattering data $T_{\mathrm{A}}^{(2)}(x)$ for $x \in U$ is a direct consequence of Lemma 3.8. 
3.3. The three-dimensional case. In this subsection, we show that the scattering data obtained from a single realization of the random source can determine uniquely the function $\phi$ in the principle symbol in the three dimensions. By (3.3) and (3.14), we have

$$
u(x, \kappa)=-\frac{1}{4 \pi} \int_{\mathbb{R}^{3}} \frac{e^{\mathrm{i} \kappa|x-y|}}{|x-y|} f(y) \mathrm{d} y,
$$

which yields

$$
\mathbb{E}\left(u\left(x, \kappa_{1}\right) \overline{u\left(x, \kappa_{2}\right)}\right)=\frac{1}{16 \pi^{2}} \int_{\mathbb{R}^{6}} \frac{e^{\mathrm{i}\left(\kappa_{1}|x-y|-\kappa_{2}|x-z|\right)}}{|x-y||x-z|} \mathbb{E}(f(y) f(z)) \mathrm{d} y \mathrm{~d} z .
$$

We apply directly Lemma 3.5 and obtain the estimates of $\mathbb{E}\left(u\left(x, \kappa_{1}\right) \overline{u\left(x, \kappa_{2}\right)}\right)$ and $\mathbb{E}\left(u\left(x, \kappa_{1}\right) u\left(x, \kappa_{2}\right)\right)$.

Lemma 3.10. For $\kappa_{1} \geq 1, \kappa_{2} \geq 1$, the following estimates

$$
\begin{aligned}
& \left|\mathbb{E}\left(u\left(x, \kappa_{1}\right) \overline{u\left(x, \kappa_{2}\right)}\right)\right| \leq c_{n}\left(\kappa_{1}+\kappa_{2}\right)^{-m}\left(1+\left|\kappa_{1}-\kappa_{2}\right|\right)^{-n}, \\
& \left|\mathbb{E}\left(u\left(x, \kappa_{1}\right) u\left(x, \kappa_{2}\right)\right)\right| \leq c_{n}\left(\kappa_{1}+\kappa_{2}\right)^{-n}\left(1+\left|\kappa_{1}-\kappa_{2}\right|\right)^{-m}
\end{aligned}
$$

holds uniformly for $x \in U$, where $n \in \mathbb{N}$ is arbitrary and $c_{n}>0$ is a constant depending only on $n$.

To derive the relationship between the scattering data and the function $\phi$ in the principle symbol, by setting $\kappa_{1}=\kappa_{2}=\kappa$ in (3.45) and using Lemma 3.7, we get

$$
\mathbb{E}\left(|u(x, \kappa)|^{2}\right)=T_{\mathrm{A}}^{(3)}(x) \kappa^{-m}+O\left(\kappa^{-(m+1)}\right),
$$

where

$$
T_{\mathrm{A}}^{(3)}(x)=\frac{C_{3}}{16 \pi^{2}} \int_{\mathbb{R}^{3}} \frac{1}{|x-y|^{2}} \phi(y) \mathrm{d} y .
$$

Now we are ready to present the main result for the three-dimensional case.

Theorem 3.11. Let the external source $f$ be a microlocally isotropic Gaussian random field which satisfies Assumption B. Then for all $x \in U$, it holds almost surely that

$$
\lim _{Q \rightarrow \infty} \frac{1}{Q-1} \int_{1}^{Q} \kappa^{m}|u(x, \kappa)|^{2} \mathrm{~d} \kappa=T_{\mathrm{A}}^{(3)}(x) .
$$

Moreover, the scattering data $T_{\mathrm{A}}^{(3)}(x), x \in U$ uniquely determines the micro-correlation strength $\phi$ through the linear integral equation (3.47).

Proof. We decompose $\kappa^{m}|u(x, \kappa)|^{2}$ into two parts:

$$
\kappa^{m}|u(x, \kappa)|^{2}=\kappa^{m} \mathbb{E}|u(x, \kappa)|^{2}+Y(x, \kappa),
$$

where

$$
Y(x, \kappa):=\kappa^{m}\left(|u(x, \kappa)|^{2}-\mathbb{E}|u(x, \kappa)|^{2}\right) .
$$

Clearly,

$$
\frac{1}{Q-1} \int_{1}^{Q} \kappa^{m}|u(x, \kappa)|^{2} \mathrm{~d} \kappa=\frac{1}{Q-1} \int_{1}^{Q} \kappa^{m} \mathbb{E}|u(x, \kappa)|^{2} \mathrm{~d} \kappa+\frac{1}{Q-1} \int_{1}^{Q} Y(x, \kappa) \mathrm{d} \kappa .
$$

Hence, (3.48) holds as long as we show that

$$
\lim _{Q \rightarrow \infty} \frac{1}{Q-1} \int_{1}^{Q} \kappa^{m} \mathbb{E}|u(x, \kappa)|^{2} \mathrm{~d} \kappa=T_{\mathrm{A}}^{(3)}(x), \quad \lim _{Q \rightarrow \infty} \frac{1}{Q-1} \int_{1}^{Q} Y(x, \kappa) \mathrm{d} \kappa=0 .
$$

The second equation in (3.49) can be obtained by a similar argument to the two-dimensional case. Using (3.46) gives

$$
\lim _{Q \rightarrow \infty} \frac{1}{Q-1} \int_{1}^{Q} \kappa^{m} \mathbb{E}|u(x, \kappa)|^{2} \mathrm{~d} \kappa=\lim _{Q \rightarrow \infty} \frac{1}{Q-1} \int_{1}^{Q}\left(T_{\mathrm{A}}^{(3)}(x)+O\left(\kappa^{-1}\right)\right) \mathrm{d} \kappa=T_{\mathrm{A}}^{(3)}(x) .
$$


Hence, the first equation in (3.49) holds which implies that (3.48) holds. A direct application of Lemma (3.8) implies that $\phi$ is uniquely determined by the scattering data $T_{\mathrm{A}}^{(3)}(x)$ for $x \in U$.

\section{Elastic Waves}

This section concerns the direct and inverse source problems for the elastic wave equation in the two- and three-dimensional cases. Following the general theme for the acoustic case presented in Section 3, we discuss the well-posedness of the direct problem and show the uniqueness of the inverse problem. We prove that the direct scattering problem with a distributional source indeed has a unique solution. For the inverse problem, we assume that each component of the external source is a microlocally isotropic Gaussian random field whose covariance operator is a classical pseudodifferential operator. Moreover, the principle symbol of the covariance operator of each component is assumed to be coincided. Our main results are as follows: in either the two- or three-dimensional case, given the scattering data which is obtained from a single realization of the random source, the principle symbol of the covariance operator can be uniquely determined. The technical details differ from acoustic waves due to the different model equation and Green tensors.

4.1. The direct scattering problem. In this subsection, we introduce the model problem of the random source scattering for elastic waves, and show that the direct problem with a distributional source is well-posed.

Consider the time-harmonic Navier equation in a homogeneous medium

$$
\mu \Delta \boldsymbol{u}+(\lambda+\mu) \nabla \nabla \cdot \boldsymbol{u}+\omega^{2} \boldsymbol{u}=\boldsymbol{f} \quad \text { in } \mathbb{R}^{d},
$$

where $\omega>0$ is the angular frequency, $\lambda$ and $\mu$ are the Lamé constants satisfying $\mu>0$ and $\lambda+\mu>0$, the external source $\boldsymbol{f} \in \mathbb{C}^{d}$ is a generalized random function supported in a bounded and simply connected domain $D$ in $\mathbb{R}^{d}$, and $\boldsymbol{u} \in \mathbb{C}^{d}$ is the displacement of the random wave field.

Since the problem is imposed in the open domain $\mathbb{R}^{d}$, an appropriate radiation condition is needed to complete the formulation of the scattering problem. We adopt the Kupradze-Sommerfeld radiation condition to describe the asymptotic behavior of the displacement field away from the source. According to the Helmholtz decomposition, the displacement $\boldsymbol{u}$ can be decomposed into the compressional part $\boldsymbol{u}_{\mathrm{p}}$ and the shear part $\boldsymbol{u}_{\mathrm{s}}$ :

$$
\boldsymbol{u}=\boldsymbol{u}_{\mathrm{p}}+\boldsymbol{u}_{\mathrm{s}} \quad \text { in } \mathbb{R}^{d} \backslash \bar{D} .
$$

The Kupradze-Sommerfeld radiation condition requires that $\boldsymbol{u}_{\mathrm{p}}$ and $\boldsymbol{u}_{\mathrm{s}}$ satisfy the Sommerfeld radiation condition:

$$
\lim _{r \rightarrow \infty} r^{\frac{d-1}{2}}\left(\partial_{r} \boldsymbol{u}_{\mathrm{p}}-\mathrm{i} \kappa_{\mathrm{p}} \boldsymbol{u}_{\mathrm{p}}\right)=0, \quad \lim _{r \rightarrow \infty} r^{\frac{d-1}{2}}\left(\partial_{r} \boldsymbol{u}_{\mathrm{s}}-\mathrm{i} \kappa_{\mathrm{s}} \boldsymbol{u}_{s}\right)=0, \quad r=|x|,
$$

where

$$
\kappa_{\mathrm{p}}=\frac{\omega}{(\lambda+2 \mu)^{1 / 2}}=c_{\mathrm{p}} \omega, \quad \kappa_{\mathrm{s}}=\frac{\omega}{\mu^{1 / 2}}=c_{\mathrm{s}} \omega
$$

are known as the compressional and shear wavenumbers with

$$
c_{\mathrm{p}}=(\lambda+2 \mu)^{-1 / 2}, \quad c_{\mathrm{s}}=\mu^{-1 / 2} .
$$

Note that $c_{\mathrm{p}}$ and $c_{\mathrm{S}}$ are independent of $\omega$ and $c_{\mathrm{p}}<c_{\mathrm{S}}$.

In (4.1), the external source $\boldsymbol{f}$ is a vector with components $f_{i}, i=1, \ldots, d$. To achieve the main results, throughout this section, we assume that each component $f_{i}$ satisfies the following condition.

Assumption C: Recall that $D \subset \mathbb{R}^{d}$ denotes a bounded and simply connected domain, $f_{i}$ is assumed to be a microlocally isotropic Gaussian random field of the same order $m \in\left[d, d+\frac{1}{2}\right)$ in $D$. Each covariance operator $C_{f_{i}}$ is assumed to have the same principle symbol $\phi(x)|\xi|^{-m}$ with $\phi \in C_{0}^{\infty}(D)$ and $\phi \geq 0$. Moreover, we assume that $\mathbb{E}\left(f_{i}\right)=0$ and $\mathbb{E}\left(f_{i} f_{j}\right)=0$ if $i \neq j$ for $i, j=1, \ldots, d$. 
According to Lemma 2.2, if $m=d$, we have $\boldsymbol{f}(\hat{\omega}) \in H^{-\varepsilon, p}(D)^{3}$. Thus it suffices to show that the scattering problem for such a deterministic, distributional source $\boldsymbol{f} \in H^{-\varepsilon, p}(D)^{3}$ has a unique solution.

Introduce the Green tensor $\boldsymbol{G}(x, y, \omega) \in \mathbb{C}^{d \times d}$ for the Navier equation (4.1) which is given by

$$
\boldsymbol{G}(x, y, \omega)=\frac{1}{\mu} \Phi_{d}\left(x, y, \kappa_{\mathrm{s}}\right) \boldsymbol{I}_{d}+\frac{1}{\omega^{2}} \nabla_{x} \nabla_{x}^{\top}\left(\Phi_{d}\left(x, y, \kappa_{\mathrm{s}}\right)-\Phi_{d}\left(x, y, \kappa_{\mathrm{p}}\right)\right),
$$

where $\boldsymbol{I}_{d}$ is the $d \times d$ identity matrix and $\Phi_{d}(x, y, \kappa)$ is the fundamental solution for the $d$-dimensional Helmholtz equation given in (3.3). Here the notation $\nabla_{x} \nabla_{x}^{\top}$ is given by

$$
\nabla_{x} \nabla_{x}^{\top} \varphi=\left[\begin{array}{ll}
\partial_{x_{1} x_{1}}^{2} \varphi & \partial_{x_{1} x_{2}}^{2} \varphi \\
\partial_{x_{2} x_{1}}^{2} \varphi & \partial_{x_{2} x_{2}}^{2} \varphi
\end{array}\right] \quad \text { if } d=2
$$

and

$$
\nabla_{x} \nabla_{x}^{\top} \varphi=\left[\begin{array}{lll}
\partial_{x_{1} x_{1}}^{2} \varphi & \partial_{x_{1} x_{2}}^{2} \varphi & \partial_{x_{1} x_{3}}^{2} \varphi \\
\partial_{x_{2} x_{1}}^{2} \varphi & \partial_{x_{2} x_{2}}^{2} \varphi & \partial_{x_{2} x_{3}}^{2} \varphi \\
\partial_{x_{3} x_{1}}^{2} \varphi & \partial_{x_{3} x_{2}}^{2} \varphi & \partial_{x_{3} x_{3}}^{2} \varphi
\end{array}\right] \quad \text { if } d=3
$$

for some scalar function $\varphi$ defined in $\mathbb{R}^{d}$. It is easily verified that the Green tensor $\boldsymbol{G}(x, y, \omega)$ is symmetric with respect to the variables $x$ and $y$.

We study the asymptotic expansion of the Green's tensor $\boldsymbol{G}(x, y, \omega)$ when $|x-y|$ is close to zero. For the two-dimensional case, using (3.4)-(3.6) gives

$$
H_{2}^{(1)}(t)=-\frac{4 \mathrm{i}}{\pi} \frac{1}{t^{2}}-\frac{\mathrm{i}}{\pi}+\frac{\mathrm{i}}{4 \pi} t^{2} \ln \frac{t}{2}+\left(\frac{\gamma \mathrm{i}}{4 \pi}-\frac{3 \mathrm{i}}{16 \pi}+\frac{1}{8}\right) t^{2}+O\left(t^{4} \ln \frac{t}{2}\right) \quad \text { as } t \rightarrow 0 .
$$

Recall the recurrence relations (3.9), a direct calculation shows that

$$
\begin{aligned}
\Phi_{2}(x, y, \kappa) & =\frac{\mathrm{i}}{4} H_{0}^{(1)}(\kappa|x-y|) \\
\partial_{x_{i}} \Phi_{2}(x, y, \kappa) & =-\frac{\kappa \mathrm{i}}{4}\left(x_{i}-y_{i}\right) \frac{H_{1}^{(1)}(\kappa|x-y|)}{|x-y|} \\
\partial_{x_{i} x_{j}}^{2} \Phi_{2}(x, y, \kappa) & =-\frac{\kappa \mathrm{i}}{4} \frac{H_{1}^{(1)}(\kappa|x-y|)}{|x-y|} \delta_{i j}+\frac{\kappa^{2} \mathrm{i}}{4}\left(x_{i}-y_{i}\right)\left(x_{j}-y_{j}\right) \frac{H_{2}^{(1)}(\kappa|x-y|)}{|x-y|^{2}}
\end{aligned}
$$

where $\delta_{i j}$ is the Kronecker delta function. Hence, by (3.8) and (4.4), we have

$$
\begin{aligned}
& \kappa_{\mathrm{S}} H_{1}^{(1)}\left(\kappa_{\mathrm{s}}|x-y|\right)-\kappa_{\mathrm{p}} H_{1}^{(1)}\left(\kappa_{\mathrm{p}}|x-y|\right)=\frac{\mathrm{i}}{\pi}|x-y|\left(\kappa_{\mathrm{s}}^{2} \ln \frac{\kappa_{\mathrm{s}}|x-y|}{2}-\kappa_{\mathrm{p}}^{2} \ln \frac{\kappa_{\mathrm{p}}|x-y|}{2}\right) \\
&+\left(\frac{1}{2}+\frac{\mathrm{i}}{\pi} \gamma-\frac{\mathrm{i}}{2 \pi}\right)\left(\kappa_{\mathrm{s}}^{2}-\kappa_{\mathrm{p}}^{2}\right)|x-y|+O\left(|x-y|^{3} \ln \frac{|x-y|}{2}\right), \\
& \kappa_{\mathrm{s}}^{2} H_{2}^{(1)}\left(\kappa_{\mathrm{s}}|x-y|\right)-\kappa_{\mathrm{p}}^{2} H_{2}^{(1)}\left(\kappa_{\mathrm{p}}|x-y|\right)=\frac{\mathrm{i}}{4 \pi}\left(\kappa_{\mathrm{s}}^{4} \ln \frac{\kappa_{\mathrm{s}}|x-y|}{2}-\kappa_{\mathrm{p}}^{4} \ln \frac{\kappa_{\mathrm{p}}|x-y|}{2}\right)|x-y|^{2} \\
&-\frac{\mathrm{i}}{\pi}\left(\kappa_{\mathrm{s}}^{2}-\kappa_{\mathrm{p}}^{2}\right)+\left(\frac{\gamma \mathrm{i}}{4 \pi}-\frac{3 \mathrm{i}}{16 \pi}+\frac{1}{8}\right)\left(\kappa_{\mathrm{s}}^{4}-\kappa_{\mathrm{p}}^{4}\right)|x-y|^{2}+O\left(|x-y|^{4} \ln \frac{|x-y|}{2}\right),
\end{aligned}
$$


which gives

$$
\begin{aligned}
\partial_{x_{i} x_{j}}^{2} & {\left[\Phi_{2}\left(x, y, \kappa_{\mathrm{s}}\right)-\Phi_{2}\left(x, y, \kappa_{\mathrm{p}}\right)\right] } \\
= & -\frac{\mathrm{i}}{4} \frac{1}{|x-y|}\left[\kappa_{\mathrm{s}} H_{1}^{(1)}\left(\kappa_{\mathrm{s}}|x-y|\right)-\kappa_{\mathrm{p}} H_{1}^{(1)}\left(\kappa_{\mathrm{p}}|x-y|\right)\right] \delta_{i j} \\
& +\frac{\mathrm{i}}{4} \frac{\left(x_{i}-y_{i}\right)\left(x_{j}-y_{j}\right)}{|x-y|^{2}}\left[\kappa_{\mathrm{s}}^{2} H_{2}^{(1)}\left(\kappa_{\mathrm{s}}|x-y|\right)-\kappa_{\mathrm{p}}^{2} H_{2}^{(1)}\left(\kappa_{\mathrm{p}}|x-y|\right)\right] \\
= & \frac{1}{4 \pi}\left(\kappa_{\mathrm{s}}^{2} \ln \frac{\kappa_{\mathrm{s}}|x-y|}{2}-\kappa_{\mathrm{p}}^{2} \ln \frac{\kappa_{\mathrm{p}}|x-y|}{2}\right) \delta_{i j}+\frac{1}{4 \pi}\left(\kappa_{\mathrm{s}}^{2}-\kappa_{\mathrm{p}}^{2}\right) \frac{\left(x_{i}-y_{i}\right)\left(x_{j}-y_{j}\right)}{|x-y|^{2}} \\
& -\frac{\mathrm{i}}{8}\left(1+\frac{2 \mathrm{i}}{\pi} \gamma-\frac{\mathrm{i}}{\pi}\right)\left(\kappa_{\mathrm{s}}^{2}-\kappa_{\mathrm{p}}^{2}\right) \delta_{i j}-\frac{1}{16 \pi}\left(x_{i}-y_{i}\right)\left(x_{j}-y_{j}\right)\left(\kappa_{\mathrm{s}}^{4} \ln \frac{\kappa_{\mathrm{s}}|x-y|}{2}-\kappa_{\mathrm{p}}^{4} \ln \frac{\kappa_{\mathrm{p}}|x-y|}{2}\right) \\
& -\frac{\mathrm{i}}{4}\left(\frac{\gamma \mathrm{i}}{4 \pi}-\frac{3 \mathrm{i}}{16 \pi}+\frac{1}{8}\right)\left(\kappa_{\mathrm{s}}^{4}-\kappa_{\mathrm{p}}^{4}\right)\left(x_{i}-y_{i}\right)\left(x_{j}-y_{j}\right)+O\left(|x-y|^{2} \ln \frac{|x-y|}{2}\right) .
\end{aligned}
$$

For the three-dimensional case, it follows from direct calculations that

$$
\begin{aligned}
\partial_{x_{i}} \Phi_{3}(x, y, \kappa)= & \frac{\left(x_{i}-y_{i}\right)}{4 \pi|x-y|^{3}} e^{\mathrm{i} \kappa|x-y|}(\mathrm{i} \kappa|x-y|-1), \\
\partial_{x_{i} x_{j}}^{2} \Phi_{3}(x, y, \kappa)= & \frac{|x-y|^{2} \delta_{i j}-3\left(x_{i}-y_{i}\right)\left(x_{j}-y_{j}\right)}{4 \pi|x-y|^{5}} e^{\mathrm{i} \kappa|x-y|}(\mathrm{i} \kappa|x-y|-1) \\
& \quad-\kappa^{2} \frac{\left(x_{i}-y_{i}\right)\left(x_{j}-y_{j}\right)}{4 \pi|x-y|^{3}} e^{\mathrm{i} \kappa|x-y|},
\end{aligned}
$$

which lead to

$$
\begin{aligned}
& \partial_{x_{i} x_{j}}^{2}\left(\Phi_{3}\left(x, y, \kappa_{\mathrm{S}}\right)-\Phi_{3}\left(x, y, \kappa_{\mathrm{p}}\right)\right) \\
& =\frac{|x-y|^{2} \delta_{i j}-3\left(x_{i}-y_{i}\right)\left(x_{j}-y_{j}\right)}{4 \pi|x-y|^{5}}\left(e^{\mathrm{i} \kappa_{\mathrm{s}}|x-y|}\left(\mathrm{i} \kappa_{\mathrm{S}}|x-y|-1\right)-e^{\mathrm{i} \kappa_{\mathrm{p}}|x-y|}\left(\mathrm{i} \kappa_{\mathrm{p}}|x-y|-1\right)\right) \\
& \quad-\frac{\left(x_{i}-y_{i}\right)\left(x_{j}-y_{j}\right)}{4 \pi|x-y|^{3}}\left(\kappa_{\mathrm{s}}^{2} e^{\mathrm{i} \kappa_{\mathrm{s}}|x-y|}-\kappa_{\mathrm{p}}^{2} e^{\mathrm{i} \kappa_{\mathrm{p}}|x-y|}\right) .
\end{aligned}
$$

Lemma 4.1. For some fixed $x \in \mathbb{R}^{d}, \boldsymbol{G}(x, \cdot, \omega) \in\left(L_{\mathrm{loc}}^{2}\left(\mathbb{R}^{d}\right) \cap H_{\mathrm{loc}}^{1, p}\left(\mathbb{R}^{d}\right)\right)^{d \times d}$, where $p \in(1,2)$ for $d=2$ and $p \in\left(1, \frac{3}{2}\right)$ for $d=3$.

Proof. For any fixed $x \in \mathbb{R}^{d}$, we choose a bounded domain $V \subset \mathbb{R}^{d}$ which contains $x$. Define $\rho:=\sup _{y \in V}|x-y|$, then we have $V \subset B_{\rho}(x)$. For the two-dimensional case, from (3.7) and (4.5), it is sufficient to show that

$$
\ln \frac{|x-y|}{2} \in L^{2}(V), \quad \frac{y_{i}-x_{i}}{|x-y|^{2}} \in L^{p}(V), \quad \forall p \in(1,2),
$$

which are proved in Lemma 3.1. For the three-dimensional case, it follows from the expansion of the exponential function $e^{t}$ that

$$
\begin{aligned}
& \kappa_{\mathrm{s}}^{2} e^{\mathrm{i} \kappa_{\mathrm{s}}|x-y|}-\kappa_{\mathrm{p}}^{2} e^{\mathrm{i} \kappa_{\mathrm{p}}|x-y|}=\left(\kappa_{\mathrm{s}}^{2}-\kappa_{\mathrm{p}}^{2}\right)+O(|x-y|), \\
& e^{\mathrm{i} \kappa_{s}|x-y|}\left(\mathrm{i} \kappa_{s}|x-y|-1\right)-e^{\mathrm{i} \kappa_{p}|x-y|}\left(\mathrm{i} \kappa_{p}|x-y|-1\right)=\frac{1}{2}\left(\kappa_{p}^{2}-\kappa_{s}^{2}\right)|x-y|^{2}+O\left(|x-y|^{3}\right) .
\end{aligned}
$$

Thus, by (3.12) and (4.6), it is sufficient to prove

$$
\frac{1}{|x-y|} \in L^{2}(V), \quad \frac{y_{i}-x_{i}}{|x-y|^{3}} \in L^{p}(V), \quad \forall p \in\left(1, \frac{3}{2}\right),
$$

which can been similarly proved to the three-dimensional case in Lemma 3.1 . 
Let $V$ and $G$ be any two bounded domains in $\mathbb{R}^{d}$. By Lemma 4.1 and the Sobolev embedding theorem, we have $\boldsymbol{G}(x, \cdot, \omega) \in\left(H^{s}(V)\right)^{d \times d}$, where $s \in(0,1)$ for $d=2$ and $s \in\left(0, \frac{1}{2}\right)$ for $d=3$. Hence, for any given $\boldsymbol{g} \in H_{0}^{-s}(V)^{d}$, in the dual sense, we define the operator $\boldsymbol{H}_{\omega}$ by

$$
\left(\boldsymbol{H}_{\omega} \boldsymbol{g}\right)(x)=\int_{V} \boldsymbol{G}(x, y, \omega) \cdot \boldsymbol{g}(y) \mathrm{d} y, \quad x \in G,
$$

where the dot is the matrix-vector multiplication. By the similar arguments to [22, Theorem 8.2], we have the following property.

Lemma 4.2. The operator $\boldsymbol{H}_{\omega}: H_{0}^{-s}(V)^{d} \rightarrow H^{s}(G)^{d}$ is bounded for $s \in(0,1), d=2$ or $s \in$ $\left(0, \frac{1}{2}\right), d=3$.

Theorem 4.3. For some fixed $s \in\left(0,1-\frac{d}{6}\right)$, assume $1<p<\frac{2 d}{d+2(1-s)}$ and $\frac{1}{p}+\frac{1}{p^{\prime}}=1$, then the scattering problem (4.1)-4.2) with the source $\boldsymbol{f} \in H_{0}^{-1, p^{\prime}}(D)^{d}$ attains a unique solution $\boldsymbol{u} \in$ $H_{\text {loc }}^{1, p}\left(\mathbb{R}^{d}\right)^{d}$ given by

$$
\boldsymbol{u}(x, \omega)=-\int_{D} \boldsymbol{G}(x, y, \omega) \cdot \boldsymbol{f}(y) \mathrm{d} y .
$$

Proof. The uniqueness of the scattering problem (4.1)-(4.2) is obvious. We focus on the existence. For convenience, we denote the differential operator in the Navier equation by

$$
\Delta^{*} \boldsymbol{u}:=\mu \Delta \boldsymbol{u}+(\lambda+\mu) \nabla \nabla \cdot \boldsymbol{u} .
$$

Let $B_{r}:=\left\{y \in \mathbb{R}^{d}:|y|<r\right\}$ be a ball which is large enough such that it contains the support of $\boldsymbol{f}$. Denote by $\nu$ the unit normal vector on the boundary $\partial B_{r}$. The generalized stress vector on $\partial B_{r}$ is defined by

$$
P \boldsymbol{u}:=\mu \partial_{\nu} \boldsymbol{u}+(\lambda+\mu)(\nabla \cdot \boldsymbol{u}) \nu .
$$

The assumption $s \in\left(0,1-\frac{d}{6}\right)$ leads to $1<\frac{2 d}{d+2(1-s)}<\frac{3}{2}$. By Lemma 4.1, we have $\boldsymbol{G}(x, \cdot, \omega) \in$ $\left(H_{\text {loc }}^{1, p}\left(\mathbb{R}^{d}\right)\right)^{d \times d}$. Since $\Delta^{*} \boldsymbol{u}+\omega^{2} \boldsymbol{u}=\boldsymbol{f} \in H_{0}^{-1, p^{\prime}}(D)^{d}$, in the sense of distributions, we have

$$
\int_{B_{r}} \boldsymbol{G}(x, y, \omega) \cdot\left(\Delta^{*} \boldsymbol{u}(y)+\omega^{2} \boldsymbol{u}(y)\right) \mathrm{d} y=\int_{B_{r}} \boldsymbol{G}(x, y, \omega) \cdot \boldsymbol{f}(y) \mathrm{d} y .
$$

Define the operator acting on $\boldsymbol{u}$ in the left-hand side of the above equation by $S_{\mathrm{E}}$. For $\varphi \in C^{\infty}\left(\mathbb{R}^{d}\right)^{d}$, from the divergence theorem, we obtain

$$
\begin{aligned}
& \left(S_{\mathrm{E}} \boldsymbol{\varphi}\right)(x)=\int_{B_{r}} \boldsymbol{G}(x, y, \omega) \cdot\left(\Delta^{*} \boldsymbol{\varphi}(y)+\omega^{2} \boldsymbol{\varphi}(y)\right) \mathrm{d} y \\
& =\int_{B_{r} \backslash B_{\delta}(x)} \boldsymbol{G}(x, y, \omega) \cdot\left(\Delta^{*} \boldsymbol{\varphi}(y)+\omega^{2} \boldsymbol{\varphi}(y)\right) \mathrm{d} y+\int_{B_{\delta}(x)} \boldsymbol{G}(x, y, \omega) \cdot\left(\Delta^{*} \boldsymbol{\varphi}(y)+\omega^{2} \boldsymbol{\varphi}(y)\right) \mathrm{d} y \\
& =\int_{B_{r} \backslash B_{\delta}(x)}\left(\boldsymbol{G}(x, y, \omega) \cdot \Delta^{*} \boldsymbol{\varphi}(y)-\Delta^{*} \boldsymbol{G}(x, y, \omega) \cdot \boldsymbol{\varphi}(y)\right) \mathrm{d} y \\
& \quad+\int_{B_{\delta}(x)} \boldsymbol{G}(x, y, \omega) \cdot\left(\Delta^{*} \boldsymbol{\varphi}(y)+\omega^{2} \boldsymbol{\varphi}(y)\right) \mathrm{d} y \\
& =\int_{B_{\delta}(x)} \boldsymbol{G}(x, y, \omega) \cdot\left(\Delta^{*} \boldsymbol{\varphi}(y)+\omega^{2} \boldsymbol{\varphi}(y)\right) \mathrm{d} y \\
& \quad+\int_{\partial B_{r} \cup \partial B_{\delta}(x)}(\boldsymbol{G}(x, y, \omega) \cdot P \boldsymbol{\varphi}(y)-P \boldsymbol{G}(x, y, \omega) \cdot \boldsymbol{\varphi}(y)) \mathrm{d} s(y),
\end{aligned}
$$

where $\delta>0$ is a sufficiently small constant. By the mean value theorem, it is easy to verify that

$$
\lim _{\delta \rightarrow 0} \int_{\partial B_{\delta}(x)}(\boldsymbol{G}(x, y, \omega) \cdot P \boldsymbol{\varphi}(y)-P \boldsymbol{G}(x, y, \omega) \cdot \boldsymbol{\varphi}(y)) \mathrm{d} s(y)=-\boldsymbol{\varphi}(x)
$$


and

$$
\lim _{\delta \rightarrow 0} \int_{B_{\delta}(x)} \boldsymbol{G}(x, y, \omega) \cdot\left(\Delta^{*} \boldsymbol{\varphi}(y)+\omega^{2} \boldsymbol{\varphi}(y)\right) \mathrm{d} y=0 .
$$

Hence, we obtain

$$
\left(\boldsymbol{S}_{\mathrm{E}} \boldsymbol{\varphi}\right)(x)=-\boldsymbol{\varphi}(x)+\int_{\partial B_{r}}(\boldsymbol{G}(x, y, \omega) \cdot P \boldsymbol{\varphi}(y)-P \boldsymbol{G}(x, y, \omega) \cdot \boldsymbol{\varphi}(y)) \mathrm{d} s(y),
$$

which implies

$$
\left(\boldsymbol{S}_{\mathrm{E}} \boldsymbol{u}\right)(x)=-\boldsymbol{u}(x)+\int_{\partial B_{r}}(\boldsymbol{G}(x, y, \omega) \cdot P \boldsymbol{u}(y)-P \boldsymbol{G}(x, y, \omega) \cdot \boldsymbol{u}(y)) \mathrm{d} s(y) .
$$

Since $\boldsymbol{u}(y)$ and $\boldsymbol{G}(x, y, \omega)$ satisfy the Sommerfeld radiation condition, we have

$$
\lim _{r \rightarrow \infty} \int_{\partial B_{r}}(\boldsymbol{G}(x, y, \omega) \cdot \boldsymbol{P} \boldsymbol{u}(y)-\boldsymbol{P} \boldsymbol{G}(x, y, \omega) \cdot \boldsymbol{u}(y)) \mathrm{d} s(y)=0 .
$$

Therefore

$$
\boldsymbol{u}(x, \omega)=-\int_{D} \boldsymbol{G}(x, y, \omega) \cdot \boldsymbol{f}(y) \mathrm{d} y=-\boldsymbol{H}_{\omega} \boldsymbol{f}(x) .
$$

Next is to show that $\boldsymbol{u} \in H_{\mathrm{loc}}^{1, p}\left(\mathbb{R}^{d}\right)^{d}$. By Lemma 4.2, we have that for $s \in\left(0,1-\frac{d}{6}\right)$, the operator $\boldsymbol{H}_{\omega}: H_{0}^{-s}(D)^{d} \rightarrow H_{\mathrm{loc}}^{s}\left(\mathbb{R}^{d}\right)^{d}$ is bounded. The assumption $1<p<\frac{2 d}{d+2(1-s)}$ implies that $\frac{1}{2}+\frac{1-s}{d}<\frac{1}{p}<1$ which yields $\frac{1}{2}-\frac{s}{d}<\frac{1}{p}-\frac{1}{d}$. Thus, the Sobolev embedding theorem implies that $H^{s}(D)$ is embedded into $H^{1, p}(D)$ and $H_{0}^{-1, p^{\prime}}(D)$ is embedded into $H_{0}^{-s}(D)$. Thus, the operator $\boldsymbol{H}_{\omega}: H_{0}^{-1, p^{\prime}}(D)^{d} \rightarrow H_{\text {loc }}^{1, p}\left(\mathbb{R}^{d}\right)^{d}$ is bounded, which completes the proof.

4.2. The two-dimensional case. This subsection is devoted to study the two-dimensional case. It is required to derive a relationship between the scattering data and the principle symbol of the covariance operator of the component of $\boldsymbol{f}$. To this end, we need to express the displacement $\boldsymbol{u}(x, \omega)$ explicitly. Substituting (4.5) into (4.3) gives that $\boldsymbol{u}(x, \omega)=\left(u_{1}(x, \omega), u_{2}(x, \omega)\right)^{\top}$ where

$$
\begin{aligned}
& u_{1}(x, \omega)=u_{11}(x, \omega)+u_{12}(x, \omega)+u_{13}(x, \omega)+u_{14}(x, \omega), \\
& u_{2}(x, \omega)=u_{21}(x, \omega)+u_{22}(x, \omega)+u_{23}(x, \omega)+u_{24}(x, \omega)
\end{aligned}
$$

where

$$
\begin{aligned}
& u_{11}(x, \omega)=\frac{\mathrm{i}}{4 \mu} \int_{D} H_{0}^{(1)}\left(\kappa_{\mathrm{s}}|x-y|\right) f_{1}(y) \mathrm{d} y, \\
& u_{12}(x, \omega)=\frac{\mathrm{i}}{4 \omega^{2}} \int_{D}\left[-\kappa_{\mathrm{s}} H_{1}^{(1)}\left(\kappa_{\mathrm{s}}|x-y|\right)+\kappa_{\mathrm{p}} H_{1}^{(1)}\left(\kappa_{\mathrm{p}}|x-y|\right)\right] \frac{1}{|x-y|} f_{1}(y) \mathrm{d} y, \\
& u_{13}(x, \omega)=\frac{\mathrm{i}}{4 \omega^{2}} \int_{D}\left[\kappa_{\mathrm{s}}^{2} H_{2}^{(1)}\left(\kappa_{\mathrm{s}}|x-y|\right)-\kappa_{\mathrm{p}}^{2} H_{2}^{(1)}\left(\kappa_{\mathrm{p}}|x-y|\right)\right] \frac{\left(x_{1}-y_{1}\right)^{2}}{|x-y|^{2}} f_{1}(y) \mathrm{d} y, \\
& u_{14}(x, \omega)=\frac{\mathrm{i}}{4 \omega^{2}} \int_{D}\left[\kappa_{\mathrm{s}}^{2} H_{2}^{(1)}\left(\kappa_{\mathrm{s}}|x-y|\right)-\kappa_{\mathrm{p}}^{2} H_{2}^{(1)}\left(\kappa_{\mathrm{p}}|x-y|\right)\right] \frac{\left(x_{1}-y_{1}\right)\left(x_{2}-y_{2}\right)}{|x-y|^{2}} f_{2}(y) \mathrm{d} y,
\end{aligned}
$$


and

$$
\begin{aligned}
& u_{21}(x, \omega)=\frac{\mathrm{i}}{4 \mu} \int_{D} H_{0}^{(1)}\left(\kappa_{\mathrm{s}}|x-y|\right) f_{2}(y) \mathrm{d} y, \\
& u_{22}(x, \omega)=\frac{\mathrm{i}}{4 \omega^{2}} \int_{D}\left[-\kappa_{\mathrm{s}} H_{1}^{(1)}\left(\kappa_{\mathrm{s}}|x-y|\right)+\kappa_{\mathrm{p}} H_{1}^{(1)}\left(\kappa_{\mathrm{p}}|x-y|\right)\right] \frac{1}{|x-y|} f_{2}(y) \mathrm{d} y, \\
& u_{23}(x, \omega)=\frac{\mathrm{i}}{4 \omega^{2}} \int_{D}\left[\kappa_{\mathrm{s}}^{2} H_{2}^{(1)}\left(\kappa_{\mathrm{s}}|x-y|\right)-\kappa_{\mathrm{p}}^{2} H_{2}^{(1)}\left(\kappa_{\mathrm{p}}|x-y|\right)\right] \frac{\left(x_{2}-y_{2}\right)^{2}}{|x-y|^{2}} f_{2}(y) \mathrm{d} y, \\
& u_{24}(x, \omega)=\frac{\mathrm{i}}{4 \omega^{2}} \int_{D}\left[\kappa_{\mathrm{s}}^{2} H_{2}^{(1)}\left(\kappa_{\mathrm{s}}|x-y|\right)-\kappa_{\mathrm{p}}^{2} H_{2}^{(1)}\left(\kappa_{\mathrm{p}}|x-y|\right)\right] \frac{\left(x_{1}-y_{1}\right)\left(x_{2}-y_{2}\right)}{|x-y|^{2}} f_{1}(y) \mathrm{d} y .
\end{aligned}
$$

To prove the main result, we need to establish the asymptotic of $\boldsymbol{u}(x, \omega)$ for $\omega \rightarrow \infty$. Recalling the definition of $H_{n, N}^{(1)}$ given in (3.17), we define $\tilde{\boldsymbol{u}}(x, \omega)=\left(\tilde{u}_{1}(x, \omega), \tilde{u}_{2}(x, \omega)\right)^{\top}$, where

$$
\begin{aligned}
& \tilde{u}_{1}(x, \omega)=\tilde{u}_{11}(x, \omega)+\tilde{u}_{12}(x, \omega)+\tilde{u}_{13}(x, \omega)+\tilde{u}_{14}(x, \omega), \\
& \tilde{u}_{2}(x, \omega)=\tilde{u}_{21}(x, \omega)+\tilde{u}_{22}(x, \omega)+\tilde{u}_{23}(x, \omega)+\tilde{u}_{24}(x, \omega) .
\end{aligned}
$$

Here

$$
\begin{aligned}
& \tilde{u}_{11}(x, \omega)=\frac{\mathrm{i}}{4 \mu} \int_{D} H_{0,2}^{(1)}\left(\kappa_{\mathrm{s}}|x-y|\right) f_{1}(y) \mathrm{d} y, \\
& \tilde{u}_{12}(x, \omega)=\frac{\mathrm{i}}{4 \omega^{2}} \int_{D}\left[-\kappa_{\mathrm{s}} H_{1,3}^{(1)}\left(\kappa_{\mathrm{s}}|x-y|\right)+\kappa_{\mathrm{p}} H_{1,3}^{(1)}\left(\kappa_{\mathrm{p}}|x-y|\right)\right] \frac{1}{|x-y|} f_{1}(y) \mathrm{d} y, \\
& \tilde{u}_{13}(x, \omega)=\frac{\mathrm{i}}{4 \omega^{2}} \int_{D}\left[\kappa_{\mathrm{s}}^{2} H_{2,4}^{(1)}\left(\kappa_{\mathrm{s}}|x-y|\right)-\kappa_{\mathrm{p}}^{2} H_{2,4}^{(1)}\left(\kappa_{\mathrm{p}}|x-y|\right)\right] \frac{\left(x_{1}-y_{1}\right)^{2}}{|x-y|^{2}} f_{1}(y) \mathrm{d} y, \\
& \tilde{u}_{14}(x, \omega)=\frac{\mathrm{i}}{4 \omega^{2}} \int_{D}\left[\kappa_{\mathrm{s}}^{2} H_{2,4}^{(1)}\left(\kappa_{\mathrm{s}}|x-y|\right)-\kappa_{\mathrm{p}}^{2} H_{2,4}^{(1)}\left(\kappa_{\mathrm{p}}|x-y|\right)\right] \frac{\left(x_{1}-y_{1}\right)\left(x_{2}-y_{2}\right)}{|x-y|^{2}} f_{2}(y) \mathrm{d} y,
\end{aligned}
$$

and

$$
\begin{aligned}
& \tilde{u}_{21}(x, \omega)=\frac{\mathrm{i}}{4 \mu} \int_{D} H_{0,2}^{(1)}\left(\kappa_{\mathrm{s}}|x-y|\right) f_{2}(y) \mathrm{d} y, \\
& \tilde{u}_{22}(x, \omega)=\frac{\mathrm{i}}{4 \omega^{2}} \int_{D}\left[-\kappa_{\mathrm{s}} H_{1,3}^{(1)}\left(\kappa_{\mathrm{s}}|x-y|\right)+\kappa_{\mathrm{p}} H_{1,3}^{(1)}\left(\kappa_{\mathrm{p}}|x-y|\right)\right] \frac{1}{|x-y|} f_{2}(y) \mathrm{d} y, \\
& \tilde{u}_{23}(x, \omega)=\frac{\mathrm{i}}{4 \omega^{2}} \int_{D}\left[\kappa_{\mathrm{s}}^{2} H_{2,4}^{(1)}\left(\kappa_{\mathrm{s}}|x-y|\right)-\kappa_{\mathrm{p}}^{2} H_{2,4}^{(1)}\left(\kappa_{\mathrm{p}}|x-y|\right)\right] \frac{\left(x_{2}-y_{2}\right)^{2}}{|x-y|^{2}} f_{2}(y) \mathrm{d} y, \\
& \tilde{u}_{24}(x, \omega)=\frac{\mathrm{i}}{4 \omega^{2}} \int_{D}\left[\kappa_{\mathrm{s}}^{2} H_{2,4}^{(1)}\left(\kappa_{\mathrm{s}}|x-y|\right)-\kappa_{\mathrm{p}}^{2} H_{2,4}^{(1)}\left(\kappa_{\mathrm{p}}|x-y|\right)\right] \frac{\left(x_{1}-y_{1}\right)\left(x_{2}-y_{2}\right)}{|x-y|^{2}} f_{1}(y) \mathrm{d} y .
\end{aligned}
$$

Lemma 4.4. The random variable $\boldsymbol{u}(x, \omega)-\tilde{\boldsymbol{u}}(x, \omega)$ satisfies almost surely the condition

$$
|\boldsymbol{u}(x, \omega)-\tilde{\boldsymbol{u}}(x, \omega)| \leq c \omega^{-\frac{7}{2}}, \quad x \in U, \quad \omega>0,
$$

where the constant $c$ depends only on $H_{0}^{-1, p^{\prime}}(D)^{2}$-norm of $\boldsymbol{f}$.

Proof. By Assumption A, it is known that there exists a positive constant $M$ such that $|x-y| \geq M$ holds for any $x \in U$ and $y \in D$. By (3.18), for $x \in U$, we have

$$
\begin{aligned}
\left|u_{11}(x, \omega)-\tilde{u}_{11}(x, \omega)\right| & =\left|\frac{\mathrm{i}}{4 \mu} \int_{D}\left[H_{0}^{(1)}\left(\kappa_{\mathrm{s}}|x-y|\right)-H_{0,2}^{(1)}\left(\kappa_{\mathrm{S}}|x-y|\right)\right] f_{1}(y) \mathrm{d} y\right| \\
& \lesssim\left\|H_{0}^{(1)}\left(\kappa_{s}|x-\cdot|\right)-H_{0,2}^{(1)}\left(\kappa_{s}|x-\cdot|\right)\right\|_{H^{1, p}(D)}\left\|f_{1}\right\|_{H_{0}^{-1, p^{\prime}}(D)} \\
& \leq c \omega^{-\frac{7}{2}}
\end{aligned}
$$

where the constant $c$ depends only on $H_{0}^{-1, p^{\prime}}(D)$-norm of $f_{1}$. 
Similarly, it is easy to verify that

$$
\left|u_{i j}(x, \omega)-\tilde{u}_{i j}(x, \omega)\right| \leq c \omega^{-\frac{7}{2}} \quad \text { for } i=1,2, j=1,2,3,4,
$$

where the constant $c$ depends only on $H_{0}^{-1, p^{\prime}}(D)^{2}$-norm of $\boldsymbol{f}$. Therefore

$$
|\boldsymbol{u}(x, \omega)-\tilde{\boldsymbol{u}}(x, \omega)| \leq \sum_{i=1}^{2} \sum_{j=1}^{4}\left|u_{i j}(x, \omega)-\tilde{u}_{i j}(x, \omega)\right| \leq c \omega^{-\frac{7}{2}},
$$

which completes the proof.

To derive the relationship between the scattering data and the function in the principle symbol, we need to estimate $\mathbb{E}\left(\boldsymbol{u}\left(x, \omega_{1}\right) \cdot \overline{\boldsymbol{u}\left(x, \omega_{2}\right)}\right)$ for $\omega_{1} \geq 1, \omega_{2} \geq 1$. By Lemma 4.4, it reduces to find the estimate of $\mathbb{E}\left(\tilde{\boldsymbol{u}}\left(x, \omega_{1}\right) \cdot \overline{\tilde{\boldsymbol{u}}\left(x, \omega_{2}\right)}\right)$ for $\omega_{1} \geq 1, \omega_{2} \geq 1$. Recalling $\tilde{\boldsymbol{u}}(x, \omega)=\left(\tilde{u}_{1}(x, \omega), \tilde{u}_{2}(x, \omega)\right)^{\top}$ and (3.17), we have

$$
\begin{aligned}
\mathbb{E}\left(\tilde{\boldsymbol{u}}\left(x, \omega_{1}\right) \cdot \overline{\tilde{\boldsymbol{u}}\left(x, \omega_{2}\right)}\right) & =\mathbb{E}\left(\tilde{u}_{1}\left(x, \omega_{1}\right) \overline{\tilde{u}_{1}\left(x, \omega_{2}\right)}\right)+\mathbb{E}\left(\tilde{u}_{2}\left(x, \omega_{1}\right) \overline{\tilde{u}_{2}\left(x, \omega_{2}\right)}\right) \\
& =\sum_{i, j=1}^{4}\left(\mathbb{E}\left(\tilde{u}_{1 i}\left(x, \omega_{1}\right) \overline{\tilde{u}_{1 j}\left(x, \omega_{2}\right)}\right)+\mathbb{E}\left(\tilde{u}_{2 i}\left(x, \omega_{1}\right) \overline{\tilde{u}_{2 j}\left(x, \omega_{2}\right)}\right)\right)
\end{aligned}
$$

where

$$
\begin{aligned}
& \tilde{u}_{11}(x, \omega)=\frac{\mathrm{i}}{4 \mu} \int_{D} H_{0,2}^{(1)}\left(\kappa_{\mathrm{s}}|x-y|\right) f_{1}(y) \mathrm{d} y \\
& =\frac{\mathrm{i}}{4 \mu} \int_{D} \kappa_{\mathrm{S}}^{-\frac{1}{2}}|x-y|^{-\frac{1}{2}} e^{\mathrm{i}\left(\kappa_{\mathrm{s}}|x-y|-\frac{1}{4} \pi\right)} \sum_{j=0}^{2} a_{j}^{(0)}\left(\frac{1}{\kappa_{\mathrm{S}}|x-y|}\right)^{j} f_{1}(y) \mathrm{d} y, \\
& \tilde{u}_{12}(x, \omega)=\frac{\mathrm{i}}{4 \omega^{2}} \int_{D}\left[-\kappa_{\mathrm{s}} H_{1,3}^{(1)}\left(\kappa_{\mathrm{s}}|x-y|\right)+\kappa_{\mathrm{p}} H_{1,3}^{(1)}\left(\kappa_{\mathrm{p}}|x-y|\right)\right] \frac{1}{|x-y|} f_{1}(y) \mathrm{d} y \\
& =\frac{\mathrm{i}}{4 \omega^{2}} \int_{D}-\kappa_{\mathrm{S}}^{\frac{1}{2}}|x-y|^{-\frac{3}{2}} e^{\mathrm{i}\left(\kappa_{\mathrm{s}}|x-y|-\frac{3}{4} \pi\right)} \sum_{j=0}^{3} a_{j}^{(1)}\left(\frac{1}{\kappa_{\mathrm{S}}|x-y|}\right)^{j} f_{1}(y) \mathrm{d} y \\
& +\frac{\mathrm{i}}{4 \omega^{2}} \int_{D} \kappa_{\mathrm{p}}^{\frac{1}{2}}|x-y|^{-\frac{3}{2}} e^{\mathrm{i}\left(\kappa_{\mathrm{p}}|x-y|-\frac{3}{4} \pi\right)} \sum_{j=0}^{3} a_{j}^{(1)}\left(\frac{1}{\kappa_{\mathrm{p}}|x-y|}\right)^{j} f_{1}(y) \mathrm{d} y, \\
& \tilde{u}_{13}(x, \omega)=\frac{\mathrm{i}}{4 \omega^{2}} \int_{D}\left[\kappa_{\mathrm{s}}^{2} H_{2,4}^{(1)}\left(\kappa_{\mathrm{s}}|x-y|\right)-\kappa_{\mathrm{p}}^{2} H_{2,4}^{(1)}\left(\kappa_{\mathrm{p}}|x-y|\right)\right] \frac{\left(x_{1}-y_{1}\right)^{2}}{|x-y|^{2}} f_{1}(y) \mathrm{d} y \\
& =\frac{\mathrm{i}}{4 \omega^{2}} \int_{D} \kappa_{\mathrm{S}}^{\frac{3}{2}}|x-y|^{-\frac{5}{2}}\left(x_{1}-y_{1}\right)^{2} e^{\mathrm{i}\left(\kappa_{\mathrm{s}}|x-y|-\frac{5}{4} \pi\right)} \sum_{j=0}^{4} a_{j}^{(2)}\left(\frac{1}{\kappa_{\mathrm{S}}|x-y|}\right)^{j} f_{1}(y) \mathrm{d} y \\
& -\frac{\mathrm{i}}{4 \omega^{2}} \int_{D} \kappa_{\mathrm{p}}^{\frac{3}{2}}|x-y|^{-\frac{5}{2}}\left(x_{1}-y_{1}\right)^{2} e^{\mathrm{i}\left(\kappa_{\mathrm{p}}|x-y|-\frac{5}{4} \pi\right)} \sum_{j=0}^{4} a_{j}^{(2)}\left(\frac{1}{\kappa_{\mathrm{p}}|x-y|}\right)^{j} f_{1}(y) \mathrm{d} y, \\
& \tilde{u}_{14}(x, \omega)=\frac{\mathrm{i}}{4 \omega^{2}} \int_{D}\left[\kappa_{\mathrm{s}}^{2} H_{2,4}^{(1)}\left(\kappa_{\mathrm{s}}|x-y|\right)-\kappa_{\mathrm{p}}^{2} H_{2,4}^{(1)}\left(\kappa_{\mathrm{p}}|x-y|\right)\right] \frac{\left(x_{1}-y_{1}\right)\left(x_{2}-y_{2}\right)}{|x-y|^{2}} f_{2}(y) \mathrm{d} y \\
& =\frac{\mathrm{i}}{4 \omega^{2}} \int_{D} \kappa_{\mathrm{S}}^{\frac{3}{2}}|x-y|^{-\frac{5}{2}}\left(x_{1}-y_{1}\right)\left(x_{2}-y_{2}\right) e^{\mathrm{i}\left(\kappa_{\mathrm{s}}|x-y|-\frac{5}{4} \pi\right)} \sum_{j=0}^{4} a_{j}^{(2)}\left(\frac{1}{\kappa_{\mathrm{S}}|x-y|}\right)^{j} f_{2}(y) \mathrm{d} y \\
& -\frac{\mathrm{i}}{4 \omega^{2}} \int_{D} \kappa_{\mathrm{p}}^{\frac{3}{2}}|x-y|^{-\frac{5}{2}}\left(x_{1}-y_{1}\right)\left(x_{2}-y_{2}\right) e^{\mathrm{i}\left(\kappa_{\mathrm{p}}|x-y|-\frac{5}{4} \pi\right)} \sum_{j=0}^{4} a_{j}^{(2)}\left(\frac{1}{\kappa_{\mathrm{p}}|x-y|}\right)^{j} f_{2}(y) \mathrm{d} y .
\end{aligned}
$$


Using the assumption $\mathbb{E}\left(f_{1} f_{2}\right)=0$, we obtain

$$
\begin{array}{ll}
\mathbb{E}\left(\tilde{u}_{11}\left(x, \omega_{1}\right) \overline{\tilde{u}_{14}\left(x, \omega_{2}\right)}\right)=0, & \mathbb{E}\left(\tilde{u}_{12}\left(x, \omega_{1}\right) \overline{\tilde{u}_{14}\left(x, \omega_{2}\right)}\right)=0, \\
\mathbb{E}\left(\tilde{u}_{13}\left(x, \omega_{1}\right) \overline{\tilde{u}_{14}\left(x, \omega_{2}\right)}\right)=0, & \mathbb{E}\left(\tilde{u}_{14}\left(x, \omega_{1}\right) \overline{\tilde{u}_{11}\left(x, \omega_{2}\right)}\right)=0, \\
\mathbb{E}\left(\tilde{u}_{14}\left(x, \omega_{1}\right) \overline{\tilde{u}_{12}\left(x, \omega_{2}\right)}\right)=0, & \mathbb{E}\left(\tilde{u}_{14}\left(x, \omega_{1}\right) \overline{\tilde{u}_{13}\left(x, \omega_{2}\right)}\right)=0,
\end{array}
$$

and

$$
\begin{aligned}
& \mathbb{E}\left(\tilde{u}_{11}\left(x, \omega_{1}\right) \overline{\tilde{u}_{11}\left(x, \omega_{2}\right)}\right) \\
& =\frac{1}{16 \mu^{2}} \sum_{j_{1}, j_{2}=0}^{2} \frac{a_{j_{1}}^{(0)} \overline{a_{j_{2}}^{(0)}}}{c_{\mathrm{S}}^{j_{1}+j_{2}+1} \omega_{1}^{j_{1}+\frac{1}{2}} \omega_{2}^{j_{1}+\frac{1}{2}}} \int_{\mathbb{R}^{4}} \frac{e^{\mathrm{i}\left(c_{\mathrm{s}} \omega_{1}|x-y|-c_{\mathrm{s}} \omega_{2}|x-z|\right)}}{|x-y|^{j_{1}+\frac{1}{2}}|x-z|^{j_{2}+\frac{1}{2}}} \mathbb{E}\left(f_{1}(y) f_{1}(z)\right) \mathrm{d} y \mathrm{~d} z, \\
& \mathbb{E}\left(\tilde{u}_{11}\left(x, \omega_{1}\right) \overline{\tilde{u}_{12}\left(x, \omega_{2}\right)}\right)=\frac{e^{\frac{\pi}{2} \mathrm{i}}}{16 \mu} \sum_{j_{1}=0}^{2} \sum_{j_{2}=0}^{3} \frac{a_{j_{1}}^{(0)} \overline{a_{j_{2}}^{(1)}}}{\omega_{1}^{j_{1}+\frac{1}{2}} \omega_{2}^{j_{2}+\frac{3}{2}}} \\
& \times \int_{\mathbb{R}^{4}}\left[-\frac{e^{\mathrm{i}\left(c_{\mathrm{s}} \omega_{1}|x-y|-c_{\mathrm{s}} \omega_{2}|x-z|\right)}}{c_{\mathrm{S}}^{j_{1}+j_{2}}}+\frac{e^{\mathrm{i}\left(c_{\mathrm{s}} \omega_{1}|x-y|-c_{\mathrm{p}} \omega_{2}|x-z|\right)}}{c_{\mathrm{s}}^{j_{1}+\frac{1}{2}} c_{\mathrm{p}}^{j_{2}-\frac{1}{2}}}\right] \frac{\mathbb{E}\left(f_{1}(y) f_{1}(z)\right)}{|x-y|^{j_{1}+\frac{1}{2}}|x-z|^{j_{2}+\frac{3}{2}}} \mathrm{~d} y \mathrm{~d} z, \\
& \mathbb{E}\left(\tilde{u}_{11}\left(x, \omega_{1}\right) \overline{\tilde{u}_{13}\left(x, \omega_{2}\right)}\right)=\frac{e^{\pi \mathrm{i}}}{16 \mu} \sum_{j_{1}=0}^{2} \sum_{j_{2}=0}^{4} \frac{a_{j_{1}}^{(0)} \overline{a_{j_{2}}^{(2)}}}{\omega_{1}^{j_{1}+\frac{1}{2}} \omega_{2}^{j_{2}+\frac{1}{2}}} \\
& \times \int_{\mathbb{R}^{4}}\left[\frac{e^{\mathrm{i}\left(c_{\mathrm{s}} \omega_{1}|x-y|-c_{\mathrm{s}} \omega_{2}|x-z|\right)}}{c_{\mathrm{S}}^{j_{1}+j_{2}-1}}-\frac{e^{\mathrm{i}\left(c_{\mathrm{s}} \omega_{1}|x-y|-c_{\mathrm{p}} \omega_{2}|x-z|\right)}}{c_{\mathrm{S}}^{j_{1}+\frac{1}{2}} c_{\mathrm{p}}^{j_{2}-\frac{3}{2}}}\right] \frac{\left(x_{1}-z_{1}\right)^{2} \mathbb{E}\left(f_{1}(y) f_{1}(z)\right)}{|x-y|^{j_{1}+\frac{1}{2}}|x-z|^{j_{2}+\frac{5}{2}}} \mathrm{~d} y \mathrm{~d} z, \\
& \mathbb{E}\left(\tilde{u}_{12}\left(x, \omega_{1}\right) \overline{\tilde{u}_{11}\left(x, \omega_{2}\right)}\right)=\frac{e^{-\frac{\pi}{2} \mathrm{i}}}{16 \mu} \sum_{j_{1}=0}^{3} \sum_{j_{2}=0}^{2} \frac{a_{j_{1}}^{(1)} \overline{a_{j_{2}}^{(0)}}}{\omega_{1}^{j_{1}+\frac{3}{2}} \omega_{2}^{j_{2}+\frac{1}{2}}} \\
& \times \int_{\mathbb{R}^{4}}\left[-\frac{e^{\mathrm{i}\left(c_{\mathrm{s}} \omega_{1}|x-y|-c_{\mathrm{s}} \omega_{2}|x-z|\right)}}{c_{\mathrm{s}}^{j_{1}+j_{2}}}+\frac{e^{\mathrm{i}\left(c_{\mathrm{p}} \omega_{1}|x-y|-c_{\mathrm{s}} \omega_{2}|x-z|\right)}}{c_{\mathrm{p}}^{j_{1}-\frac{1}{2}} c_{\mathrm{s}}^{j_{2}+\frac{1}{2}}}\right] \frac{\mathbb{E}\left(f_{1}(y) f_{1}(z)\right)}{|x-y|^{j_{1}+\frac{3}{2}}|x-z|^{j_{2}+\frac{1}{2}}} \mathrm{~d} y \mathrm{~d} z, \\
& \mathbb{E}\left(\tilde{u}_{12}\left(x, \omega_{1}\right) \overline{\tilde{u}_{12}\left(x, \omega_{2}\right)}\right)=\frac{1}{16} \sum_{j_{1}, j_{2}=0}^{3} \frac{a_{j_{1}}^{(1)} \overline{a_{j_{2}}^{(1)}}}{\omega_{1}^{j_{1}+\frac{3}{2}} \omega_{2}^{j_{2}+\frac{3}{2}}} \\
& \times \int_{\mathbb{R}^{4}}\left[\frac{e^{\mathrm{i}\left(c_{\mathrm{s}} \omega_{1}|x-y|-c_{\mathrm{s}} \omega_{2}|x-z|\right)}}{c_{\mathrm{s}}^{j_{1}+j_{2}-1}}+\frac{e^{\mathrm{i}\left(c_{\mathrm{p}} \omega_{1}|x-y|-c_{\mathrm{p}} \omega_{2}|x-z|\right)}}{c_{\mathrm{p}}^{j_{1}+j_{2}-1}}\right. \\
& \left.-\frac{e^{\mathrm{i}\left(c_{\mathrm{s}} \omega_{1}|x-y|-c_{\mathrm{p}} \omega_{2}|x-z|\right)}}{c_{\mathrm{S}}^{j_{1}-\frac{1}{2}} c_{\mathrm{p}}^{j_{2}-\frac{1}{2}}}-\frac{e^{\mathrm{i}\left(c_{\mathrm{p}} \omega_{1}|x-y|-c_{\mathrm{s}} \omega_{2}|x-z|\right)}}{c_{\mathrm{p}}^{j_{1}-\frac{1}{2}} c_{\mathrm{S}}^{j_{2}-\frac{1}{2}}}\right] \frac{\mathbb{E}\left(f_{1}(y) f_{1}(z)\right)}{|x-y|^{j_{1}+\frac{3}{2}}|x-z|^{j_{2}+\frac{3}{2}}} \mathrm{~d} y \mathrm{~d} z,
\end{aligned}
$$




$$
\begin{aligned}
& \mathbb{E}\left(\tilde{u}_{12}\left(x, \omega_{1}\right) \overline{\tilde{u}_{13}\left(x, \omega_{2}\right)}\right)=\frac{e^{\frac{\pi}{2} \mathrm{i}}}{16} \sum_{j_{1}=0}^{3} \sum_{j_{2}=0}^{4} \frac{a_{j_{1}}^{(1)} \overline{a_{j_{2}}^{(2)}}}{\omega_{1}^{j_{1}+\frac{3}{2}} \omega_{2}^{j_{2}+\frac{1}{2}}} \\
& \times \int_{\mathbb{R}^{4}}\left[-\frac{e^{\mathrm{i}\left(c_{\mathrm{s}} \omega_{1}|x-y|-c_{\mathrm{s}} \omega_{2}|x-z|\right)}}{c_{\mathrm{S}}^{j_{1}+j_{2}-2}}-\frac{e^{\mathrm{i}\left(c_{\mathrm{p}} \omega_{1}|x-y|-c_{\mathrm{p}} \omega_{2}|x-z|\right)}}{c_{\mathrm{p}}^{j_{1}+j_{2}-2}}\right. \\
& \left.+\frac{e^{\mathrm{i}\left(c_{\mathrm{s}} \omega_{1}|x-y|-c_{\mathrm{p}} \omega_{2}|x-z|\right)}}{c_{\mathrm{s}}^{j_{1}-\frac{1}{2}} c_{\mathrm{p}}^{j_{2}-\frac{3}{2}}}+\frac{e^{\mathrm{i}\left(c_{\mathrm{p}} \omega_{1}|x-y|-c_{\mathrm{s}} \omega_{2}|x-z|\right)}}{c_{\mathrm{p}}^{j_{1}-\frac{1}{2}} c_{\mathrm{s}}^{j_{2}-\frac{3}{2}}}\right] \frac{\left(x_{1}-z_{1}\right)^{2} \mathbb{E}\left(f_{1}(y) f_{1}(z)\right)}{|x-y|^{j_{1}+\frac{3}{2}}|x-z|^{j_{2}+\frac{5}{2}}} \mathrm{~d} y \mathrm{~d} z, \\
& \mathbb{E}\left(\tilde{u}_{13}\left(x, \omega_{1}\right) \overline{\tilde{u}_{11}\left(x, \omega_{2}\right)}\right)=\frac{e^{-\pi \mathrm{i}}}{16 \mu} \sum_{j_{1}=0}^{4} \sum_{j_{2}=0}^{2} \frac{a_{j_{1}}^{(2)} \overline{a_{j_{2}}^{(0)}}}{\omega_{1}^{j_{1}+\frac{1}{2}} \omega_{2}^{j_{2}+\frac{1}{2}}} \\
& \times \int_{\mathbb{R}^{4}}\left[\frac{e^{\mathrm{i}\left(c_{\mathrm{s}} \omega_{1}|x-y|-c_{\mathrm{s}} \omega_{2}|x-z|\right)}}{c_{\mathrm{S}}^{j_{1}+j_{2}-1}}-\frac{e^{\mathrm{i}\left(c_{\mathrm{p}} \omega_{1}|x-y|-c_{\mathrm{s}} \omega_{2}|x-z|\right)}}{c_{\mathrm{p}}^{j_{1}-\frac{3}{2}} c_{\mathrm{S}}^{j_{2}+\frac{1}{2}}}\right] \frac{\left(x_{1}-y_{1}\right)^{2} \mathbb{E}\left(f_{1}(y) f_{1}(z)\right)}{|x-y|^{j_{1}+\frac{5}{2}}|x-z|^{j_{2}+\frac{1}{2}}} \mathrm{~d} y \mathrm{~d} z, \\
& \mathbb{E}\left(\tilde{u}_{13}\left(x, \omega_{1}\right) \overline{\tilde{u}_{12}\left(x, \omega_{2}\right)}\right)=\frac{e^{-\frac{\pi}{2} \mathrm{i}}}{16} \sum_{j_{1}=0}^{4} \sum_{j_{2}=0}^{3} \frac{a_{j_{1}}^{(2)} \overline{a_{j_{2}}^{(1)}}}{\omega_{1}^{j_{1}+\frac{1}{2}} \omega_{2}^{j_{2}+\frac{3}{2}}} \\
& \times \int_{\mathbb{R}^{4}}\left[-\frac{e^{\mathrm{i}\left(c_{\mathrm{s}} \omega_{1}|x-y|-c_{\mathrm{s}} \omega_{2}|x-z|\right)}}{c_{\mathrm{S}}^{j_{1}+j_{2}-2}}-\frac{e^{\mathrm{i}\left(c_{\mathrm{p}} \omega_{1}|x-y|-c_{\mathrm{p}} \omega_{2}|x-z|\right)}}{c_{\mathrm{p}}^{j_{1}+j_{2}-2}}\right. \\
& \left.+\frac{e^{\mathrm{i}\left(c_{\mathrm{s}} \omega_{1}|x-y|-c_{\mathrm{p}} \omega_{2}|x-z|\right)}}{c_{\mathrm{S}}^{j_{1}-\frac{3}{2}} c_{\mathrm{p}}^{j_{2}-\frac{1}{2}}}+\frac{e^{\mathrm{i}\left(c_{\mathrm{p}} \omega_{1}|x-y|-c_{\mathrm{s}} \omega_{2}|x-z|\right)}}{c_{\mathrm{p}}^{j_{1}-\frac{3}{2}} c_{\mathrm{S}}^{j_{2}-\frac{1}{2}}}\right] \frac{\left(x_{1}-y_{1}\right)^{2} \mathbb{E}\left(f_{1}(y) f_{1}(z)\right)}{|x-y|^{j_{1}+\frac{5}{2}}|x-z|^{j_{2}+\frac{3}{2}}} \mathrm{~d} y \mathrm{~d} z, \\
& \mathbb{E}\left(\tilde{u}_{13}\left(x, \omega_{1}\right) \overline{\tilde{u}_{13}\left(x, \omega_{2}\right)}\right)=\frac{1}{16} \sum_{j_{1}, j_{2}=0}^{4} \frac{a_{j_{1}}^{(2)} \overline{a_{j_{2}}^{(2)}}}{\omega_{1}^{j_{1}+\frac{1}{2}} \omega_{2}^{j_{2}+\frac{1}{2}}} \\
& \times \int_{\mathbb{R}^{4}}\left[\frac{e^{\mathrm{i}\left(c_{\mathrm{s}} \omega_{1}|x-y|-c_{\mathrm{s}} \omega_{2}|x-z|\right)}}{c_{\mathrm{S}}^{j_{1}+j_{2}-3}}+\frac{e^{\mathrm{i}\left(c_{\mathrm{p}} \omega_{1}|x-y|-c_{\mathrm{p}} \omega_{2}|x-z|\right)}}{c_{\mathrm{p}}^{j_{1}+j_{2}-3}}\right. \\
& \left.-\frac{e^{\mathrm{i}\left(c_{\mathrm{s}} \omega_{1}|x-y|-c_{\mathrm{p}} \omega_{2}|x-z|\right)}}{c_{\mathrm{s}}^{j_{1}-\frac{3}{2}} c_{\mathrm{p}}^{j_{2}-\frac{3}{2}}}-\frac{e^{\mathrm{i}\left(c_{\mathrm{p}} \omega_{1}|x-y|-c_{\mathrm{s}} \omega_{2}|x-z|\right)}}{c_{\mathrm{p}}^{j_{1}-\frac{3}{2}} c_{\mathrm{s}}^{j_{2}-\frac{3}{2}}}\right] \frac{\left(x_{1}-y_{1}\right)^{2}\left(x_{1}-z_{1}\right)^{2} \mathbb{E}\left(f_{1}(y) f_{1}(z)\right)}{|x-y|^{j_{1}+\frac{5}{2}}|x-z|^{j_{2}+\frac{5}{2}}} \mathrm{~d} y \mathrm{~d} z, \\
& \mathbb{E}\left(\tilde{u}_{14}\left(x, \omega_{1}\right) \overline{\tilde{u}_{14}\left(x, \omega_{2}\right)}\right)=\frac{1}{16} \sum_{j_{1}, j_{2}=0}^{4} \frac{a_{j_{1}}^{(2)} \overline{a_{j_{2}}^{(2)}}}{\omega_{1}^{j_{1}+\frac{1}{2}} \omega_{2}^{j_{2}+\frac{1}{2}}} \\
& \times \int_{\mathbb{R}^{4}}\left[\frac{e^{\mathrm{i}\left(c_{\mathrm{s}} \omega_{1}|x-y|-c_{\mathrm{s}} \omega_{2}|x-z|\right)}}{c_{\mathrm{S}}^{j_{1}+j_{2}-3}}+\frac{e^{\mathrm{i}\left(c_{\mathrm{p}} \omega_{1}|x-y|-c_{\mathrm{p}} \omega_{2}|x-z|\right)}}{c_{\mathrm{p}}^{j_{1}+j_{2}-3}}-\frac{e^{\mathrm{i}\left(c_{\mathrm{s}} \omega_{1}|x-y|-c_{\mathrm{p}} \omega_{2}|x-z|\right)}}{c_{\mathrm{S}}^{j_{1}-\frac{3}{2}} c_{\mathrm{p}}^{j_{2}-\frac{3}{2}}}\right. \\
& \left.-\frac{e^{\mathrm{i}\left(c_{\mathrm{p}} \omega_{1}|x-y|-c_{\mathrm{s}} \omega_{2}|x-z|\right)}}{c_{\mathrm{p}}^{j_{1}-\frac{3}{2}} c_{\mathrm{s}}^{j_{2}-\frac{3}{2}}}\right] \frac{\left(x_{1}-y_{1}\right)\left(x_{2}-y_{2}\right)\left(x_{1}-z_{1}\right)\left(x_{2}-z_{2}\right)}{|x-y|^{j_{1}+\frac{5}{2}}|x-z|^{j_{2}+\frac{5}{2}}} \mathbb{E}\left(f_{2}(y) f_{2}(z)\right) \mathrm{d} y \mathrm{~d} z,
\end{aligned}
$$


For the second component $\tilde{u}_{2}(x, \omega)$, we have from (3.17) that

$$
\begin{aligned}
& \tilde{u}_{21}(x, \omega)=\frac{\mathrm{i}}{4 \mu} \int_{D} H_{0,2}^{(1)}\left(\kappa_{\mathrm{s}}|x-y|\right) f_{2}(y) d y \\
& =\frac{\mathrm{i}}{4 \mu} \int_{D} \kappa_{\mathrm{S}}^{-\frac{1}{2}}|x-y|^{-\frac{1}{2}} e^{\mathrm{i}\left(\kappa_{\mathrm{s}}|x-y|-\frac{1}{4} \pi\right)} \sum_{j=0}^{2} a_{j}^{(0)}\left(\frac{1}{\kappa_{\mathrm{S}}|x-y|}\right)^{j} f_{2}(y) \mathrm{d} y, \\
& \tilde{u}_{22}(x, \omega)=\frac{\mathrm{i}}{4 \omega^{2}} \int_{D}\left[-\kappa_{\mathrm{s}} H_{1,3}^{(1)}\left(\kappa_{\mathrm{s}}|x-y|\right)+\kappa_{\mathrm{p}} H_{1,3}^{(1)}\left(\kappa_{\mathrm{p}}|x-y|\right)\right] \frac{1}{|x-y|} f_{2}(y) \mathrm{d} y \\
& =\frac{\mathrm{i}}{4 \omega^{2}} \int_{D}-\kappa_{\mathrm{S}}^{\frac{1}{2}}|x-y|^{-\frac{3}{2}} e^{\mathrm{i}\left(\kappa_{\mathrm{s}}|x-y|-\frac{3}{4} \pi\right)} \sum_{j=0}^{3} a_{j}^{(1)}\left(\frac{1}{\kappa_{\mathrm{S}}|x-y|}\right)^{j} f_{2}(y) \mathrm{d} y \\
& +\frac{\mathrm{i}}{4 \omega^{2}} \int_{D} \kappa_{\mathrm{p}}^{\frac{1}{2}}|x-y|^{-\frac{3}{2}} e^{\mathrm{i}\left(\kappa_{\mathrm{p}}|x-y|-\frac{3}{4} \pi\right)} \sum_{j=0}^{3} a_{j}^{(1)}\left(\frac{1}{\kappa_{\mathrm{p}}|x-y|}\right)^{j} f_{2}(y) \mathrm{d} y, \\
& \tilde{u}_{23}(x, \omega)=\frac{\mathrm{i}}{4 \omega^{2}} \int_{D}\left[\kappa_{\mathrm{s}}^{2} H_{2,4}^{(1)}\left(\kappa_{\mathrm{s}}|x-y|\right)-\kappa_{\mathrm{p}}^{2} H_{2,4}^{(1)}\left(\kappa_{\mathrm{p}}|x-y|\right)\right] \frac{\left(x_{2}-y_{2}\right)^{2}}{|x-y|^{2}} f_{2}(y) \mathrm{d} y \\
& =\frac{\mathrm{i}}{4 \omega^{2}} \int_{D} \kappa_{\mathrm{S}}^{\frac{3}{2}}|x-y|^{-\frac{5}{2}}\left(x_{2}-y_{2}\right)^{2} e^{\mathrm{i}\left(\kappa_{\mathrm{s}}|x-y|-\frac{5}{4} \pi\right)} \sum_{j=0}^{4} a_{j}^{(2)}\left(\frac{1}{\kappa_{\mathrm{S}}|x-y|}\right)^{j} f_{2}(y) \mathrm{d} y \\
& -\frac{\mathrm{i}}{4 \omega^{2}} \int_{D} \kappa_{\mathrm{p}}^{\frac{3}{2}}|x-y|^{-\frac{5}{2}}\left(x_{2}-y_{2}\right)^{2} e^{\mathrm{i}\left(\kappa_{\mathrm{p}}|x-y|-\frac{5}{4} \pi\right)} \sum_{j=0}^{4} a_{j}^{(2)}\left(\frac{1}{\kappa_{\mathrm{p}}|x-y|}\right)^{j} f_{2}(y) \mathrm{d} y, \\
& \tilde{u}_{24}(x, \omega)=\frac{\mathrm{i}}{4 \omega^{2}} \int_{D}\left[\kappa_{\mathrm{s}}^{2} H_{2,4}^{(1)}\left(\kappa_{\mathrm{s}}|x-y|\right)-\kappa_{\mathrm{p}}^{2} H_{2,4}^{(1)}\left(\kappa_{\mathrm{p}}|x-y|\right)\right] \frac{\left(x_{1}-y_{1}\right)\left(x_{2}-y_{2}\right)}{|x-y|^{2}} f_{1}(y) \mathrm{d} y \\
& =\frac{\mathrm{i}}{4 \omega^{2}} \int_{D} \kappa_{\mathrm{S}}^{\frac{3}{2}}|x-y|^{-\frac{5}{2}}\left(x_{1}-y_{1}\right)\left(x_{2}-y_{2}\right) e^{\mathrm{i}\left(\kappa_{\mathrm{s}}|x-y|-\frac{5}{4} \pi\right)} \sum_{j=0}^{4} a_{j}^{(2)}\left(\frac{1}{\kappa_{\mathrm{S}}|x-y|}\right)^{j} f_{1}(y) \mathrm{d} y \\
& -\frac{\mathrm{i}}{4 \omega^{2}} \int_{D} \kappa_{\mathrm{p}}^{\frac{3}{2}}|x-y|^{-\frac{5}{2}}\left(x_{1}-y_{1}\right)\left(x_{2}-y_{2}\right) e^{\mathrm{i}\left(\kappa_{\mathrm{p}}|x-y|-\frac{5}{4} \pi\right)} \sum_{j=0}^{4} a_{j}^{(2)}\left(\frac{1}{\kappa_{\mathrm{p}}|x-y|}\right)^{j} f_{1}(y) \mathrm{d} y .
\end{aligned}
$$

Noting $\mathbb{E}\left(f_{1} f_{2}\right)=0$ from Assumption C, we deduce that

$$
\begin{array}{ll}
\mathbb{E}\left(\tilde{u}_{21}\left(x, \omega_{1}\right) \overline{\tilde{u}_{24}\left(x, \omega_{2}\right)}\right)=0, & \mathbb{E}\left(\tilde{u}_{22}\left(x, \omega_{1}\right) \overline{\tilde{u}_{24}\left(x, \omega_{2}\right)}\right)=0, \\
\mathbb{E}\left(\tilde{u}_{23}\left(x, \omega_{1}\right) \overline{\tilde{u}_{24}\left(x, \omega_{2}\right)}\right)=0, & \mathbb{E}\left(\tilde{u}_{24}\left(x, \omega_{1}\right) \overline{\tilde{u}_{21}\left(x, \omega_{2}\right)}\right)=0, \\
\mathbb{E}\left(\tilde{u}_{24}\left(x, \omega_{1}\right) \overline{\tilde{u}_{22}\left(x, \omega_{2}\right)}\right)=0, & \mathbb{E}\left(\tilde{u}_{24}\left(x, \omega_{1}\right) \overline{\tilde{u}_{23}\left(x, \omega_{2}\right)}\right)=0,
\end{array}
$$

and

$$
\begin{aligned}
& \mathbb{E}\left(\tilde{u}_{21}\left(x, \omega_{1}\right) \overline{\tilde{u}_{21}\left(x, \omega_{2}\right)}\right) \\
& =\frac{1}{16 \mu^{2}} \sum_{j_{1}, j_{2}=0}^{2} \frac{a_{j_{1}}^{(0)} \overline{a_{j_{2}}^{(0)}}}{c_{\mathrm{s}}^{j_{1}+j_{2}+1} \omega_{1}^{j_{1}+\frac{1}{2}} \omega_{2}^{j_{1}+\frac{1}{2}}} \int_{\mathbb{R}^{4}} \frac{e^{\mathrm{i}\left(c_{\mathrm{s}} \omega_{1}|x-y|-c_{\mathrm{s}} \omega_{2}|x-z|\right)}}{|x-y|^{j_{1}+\frac{1}{2}}|x-z|^{j_{2}+\frac{1}{2}}} \mathbb{E}\left(f_{2}(y) f_{2}(z)\right) \mathrm{d} y \mathrm{~d} z,
\end{aligned}
$$




$$
\begin{aligned}
& \mathbb{E}\left(\tilde{u}_{21}\left(x, \omega_{1}\right) \overline{\tilde{u}_{22}\left(x, \omega_{2}\right)}\right)=\frac{e^{\frac{\pi}{2} \mathrm{i}}}{16 \mu} \sum_{j_{1}=0}^{2} \sum_{j_{2}=0}^{3} \frac{a_{j_{1}}^{(0)} \overline{a_{j_{2}}^{(1)}}}{\omega_{1}^{j_{1}+\frac{1}{2}} \omega_{2}^{j_{2}+\frac{3}{2}}} \\
& \times \int_{\mathbb{R}^{4}}\left[-\frac{e^{\mathrm{i}\left(c_{\mathrm{s}} \omega_{1}|x-y|-c_{\mathrm{s}} \omega_{2}|x-z|\right)}}{c_{\mathrm{S}}^{j_{1}+j_{2}}}+\frac{e^{\mathrm{i}\left(c_{\mathrm{s}} \omega_{1}|x-y|-c_{\mathrm{p}} \omega_{2}|x-z|\right)}}{c_{\mathrm{s}}^{j_{1}+\frac{1}{2}} c_{\mathrm{p}}^{j_{2}-\frac{1}{2}}}\right] \frac{\mathbb{E}\left(f_{2}(y) f_{2}(z)\right)}{|x-y|^{j_{1}+\frac{1}{2}}|x-z|^{j_{2}+\frac{3}{2}}} \mathrm{~d} y \mathrm{~d} z, \\
& \mathbb{E}\left(\tilde{u}_{21}\left(x, \omega_{1}\right) \overline{\tilde{u}_{23}\left(x, \omega_{2}\right)}\right)=\frac{e^{\pi \mathrm{i}}}{16 \mu} \sum_{j_{1}=0}^{2} \sum_{j_{2}=0}^{4} \frac{a_{j_{1}}^{(0)} \overline{a_{j_{2}}^{(2)}}}{\omega_{1}^{j_{1}+\frac{1}{2}} \omega_{2}^{j_{2}+\frac{1}{2}}} \\
& \times \int_{\mathbb{R}^{4}}\left[\frac{e^{\mathrm{i}\left(c_{\mathrm{s}} \omega_{1}|x-y|-c_{\mathrm{s}} \omega_{2}|x-z|\right)}}{c_{\mathrm{S}}^{j_{1}+j_{2}-1}}-\frac{e^{\mathrm{i}\left(c_{\mathrm{s}} \omega_{1}|x-y|-c_{\mathrm{p}} \omega_{2}|x-z|\right)}}{c_{\mathrm{S}}^{j_{1}+\frac{1}{2}} c_{\mathrm{p}}^{j_{2}-\frac{3}{2}}}\right] \frac{\left(x_{2}-z_{2}\right)^{2} \mathbb{E}\left(f_{2}(y) f_{2}(z)\right)}{|x-y|^{j_{1}+\frac{1}{2}}|x-z|^{j_{2}+\frac{5}{2}}} \mathrm{~d} y \mathrm{~d} z, \\
& \mathbb{E}\left(\tilde{u}_{22}\left(x, \omega_{1}\right) \overline{\tilde{u}_{21}\left(x, \omega_{2}\right)}\right)=\frac{e^{-\frac{\pi}{2} \mathrm{i}}}{16 \mu} \sum_{j_{1}=0}^{3} \sum_{j_{2}=0}^{2} \frac{a_{j_{1}}^{(1)} \overline{a_{j_{2}}^{(0)}}}{\omega_{1}^{j_{1}+\frac{3}{2}} \omega_{2}^{j_{2}+\frac{1}{2}}} \\
& \times \int_{\mathbb{R}^{4}}\left[-\frac{e^{\mathrm{i}\left(c_{\mathrm{s}} \omega_{1}|x-y|-c_{\mathrm{s}} \omega_{2}|x-z|\right)}}{c_{\mathrm{S}}^{j_{1}+j_{2}}}+\frac{e^{\mathrm{i}\left(c_{\mathrm{p}} \omega_{1}|x-y|-c_{\mathrm{s}} \omega_{2}|x-z|\right)}}{c_{\mathrm{p}}^{j_{1}-\frac{1}{2}} c_{\mathrm{s}}^{j_{2}+\frac{1}{2}}}\right] \frac{\mathbb{E}\left(f_{2}(y) f_{2}(z)\right)}{|x-y|^{j_{1}+\frac{3}{2}}|x-z|^{j_{2}+\frac{1}{2}}} \mathrm{~d} y \mathrm{~d} z, \\
& \mathbb{E}\left(\tilde{u}_{22}\left(x, \omega_{1}\right) \overline{\tilde{u}_{22}\left(x, \omega_{2}\right)}\right)=\frac{1}{16} \sum_{j_{1}, j_{2}=0}^{3} \frac{a_{j_{1}}^{(1)} \overline{a_{j_{2}}^{(1)}}}{\omega_{1}^{j_{1}+\frac{3}{2}} \omega_{2}^{j_{2}+\frac{3}{2}}} \\
& \times \int_{\mathbb{R}^{4}}\left[\frac{e^{\mathrm{i}\left(c_{\mathrm{s}} \omega_{1}|x-y|-c_{\mathrm{s}} \omega_{2}|x-z|\right)}}{c_{\mathrm{s}}^{j_{1}+j_{2}-1}}+\frac{e^{\mathrm{i}\left(c_{\mathrm{p}} \omega_{1}|x-y|-c_{\mathrm{p}} \omega_{2}|x-z|\right)}}{c_{\mathrm{p}}^{j_{1}+j_{2}-1}}\right. \\
& \left.-\frac{e^{\mathrm{i}\left(c_{\mathrm{s}} \omega_{1}|x-y|-c_{\mathrm{p}} \omega_{2}|x-z|\right)}}{c_{\mathrm{S}}^{j_{1}-\frac{1}{2}} c_{\mathrm{p}}^{j_{2}-\frac{1}{2}}}-\frac{e^{\mathrm{i}\left(c_{\mathrm{p}} \omega_{1}|x-y|-c_{\mathrm{s}} \omega_{2}|x-z|\right)}}{c_{\mathrm{p}}^{j_{1}-\frac{1}{2}} c_{\mathrm{S}}^{j_{2}-\frac{1}{2}}}\right] \frac{\mathbb{E}\left(f_{2}(y) f_{2}(z)\right)}{|x-y|^{j_{1}+\frac{3}{2}}|x-z|^{j_{2}+\frac{3}{2}}} \mathrm{~d} y \mathrm{~d} z, \\
& \mathbb{E}\left(\tilde{u}_{22}\left(x, \omega_{1}\right) \overline{\tilde{u}_{23}\left(x, \omega_{2}\right)}\right)=\frac{e^{\frac{\pi}{2} \mathrm{i}}}{16} \sum_{j_{1}=0}^{3} \sum_{j_{2}=0}^{4} \frac{a_{j_{1}}^{(1)} \overline{a_{j_{2}}^{(2)}}}{\omega_{1}^{j_{1}+\frac{3}{2}} \omega_{2}^{j_{2}+\frac{1}{2}}} \\
& \times \int_{\mathbb{R}^{4}}\left[-\frac{e^{\mathrm{i}\left(c_{\mathrm{s}} \omega_{1}|x-y|-c_{\mathrm{s}} \omega_{2}|x-z|\right)}}{c_{\mathrm{s}}^{j_{1}+j_{2}-2}}-\frac{e^{\mathrm{i}\left(c_{\mathrm{p}} \omega_{1}|x-y|-c_{\mathrm{p}} \omega_{2}|x-z|\right)}}{c_{\mathrm{p}}^{j_{1}+j_{2}-2}}\right. \\
& \left.+\frac{e^{\mathrm{i}\left(c_{\mathrm{s}} \omega_{1}|x-y|-c_{\mathrm{p}} \omega_{2}|x-z|\right)}}{c_{\mathrm{S}}^{j_{1}-\frac{1}{2}} c_{\mathrm{p}}^{j_{2}-\frac{3}{2}}}+\frac{e^{\mathrm{i}\left(c_{\mathrm{p}} \omega_{1}|x-y|-c_{\mathrm{s}} \omega_{2}|x-z|\right)}}{c_{\mathrm{p}}^{j_{1}-\frac{1}{2}} c_{\mathrm{S}}^{j_{2}-\frac{3}{2}}}\right] \frac{\left(x_{2}-z_{2}\right)^{2} \mathbb{E}\left(f_{2}(y) f_{2}(z)\right)}{|x-y|^{j_{1}+\frac{3}{2}}|x-z|^{j_{2}+\frac{5}{2}}} \mathrm{~d} y \mathrm{~d} z, \\
& \mathbb{E}\left(\tilde{u}_{23}\left(x, \omega_{1}\right) \overline{\tilde{u}_{21}\left(x, \omega_{2}\right)}\right)=\frac{e^{-\pi \mathrm{i}}}{16 \mu} \sum_{j_{1}=0}^{4} \sum_{j_{2}=0}^{2} \frac{a_{j_{1}}^{(2)} \overline{a_{j_{2}}^{(0)}}}{\omega_{1}^{j_{1}+\frac{1}{2}} \omega_{2}^{j_{2}+\frac{1}{2}}} \\
& \times \int_{\mathbb{R}^{4}}\left[\frac{e^{\mathrm{i}\left(c_{\mathrm{s}} \omega_{1}|x-y|-c_{\mathrm{s}} \omega_{2}|x-z|\right)}}{c_{\mathrm{S}}^{j_{1}+j_{2}-1}}-\frac{e^{\mathrm{i}\left(c_{\mathrm{p}} \omega_{1}|x-y|-c_{\mathrm{s}} \omega_{2}|x-z|\right)}}{c_{\mathrm{p}}^{j_{1}-\frac{3}{2}} c_{\mathrm{s}}^{j_{2}+\frac{1}{2}}}\right] \frac{\left(x_{2}-y_{2}\right)^{2} \mathbb{E}\left(f_{2}(y) f_{2}(z)\right)}{|x-y|^{j_{1}+\frac{5}{2}}|x-z|^{j_{2}+\frac{1}{2}}} \mathrm{~d} y \mathrm{~d} z,
\end{aligned}
$$




$$
\begin{aligned}
& \mathbb{E}\left(\tilde{u}_{23}\left(x, \omega_{1}\right) \overline{\tilde{u}_{22}\left(x, \omega_{2}\right)}\right)=\frac{e^{-\frac{\pi}{2} \mathrm{i}}}{16} \sum_{j_{1}=0}^{4} \sum_{j_{2}=0}^{3} \frac{a_{j_{1}}^{(2)} \overline{a_{j_{2}}^{(1)}}}{\omega_{1}^{j_{1}+\frac{1}{2}} \omega_{2}^{j_{2}+\frac{3}{2}}} \\
& \times \int_{\mathbb{R}^{4}}\left[-\frac{e^{\mathrm{i}\left(c_{\mathrm{s}} \omega_{1}|x-y|-c_{\mathrm{s}} \omega_{2}|x-z|\right)}}{c_{\mathrm{S}}^{j_{1}+j_{2}-2}}-\frac{e^{\mathrm{i}\left(c_{\mathrm{p}} \omega_{1}|x-y|-c_{\mathrm{p}} \omega_{2}|x-z|\right)}}{c_{\mathrm{p}}^{j_{1}+j_{2}-2}}\right. \\
& \left.+\frac{e^{\mathrm{i}\left(c_{\mathrm{s}} \omega_{1}|x-y|-c_{\mathrm{p}} \omega_{2}|x-z|\right)}}{c_{\mathrm{S}}^{j_{1}-\frac{3}{2}} c_{\mathrm{p}}^{j_{2}-\frac{1}{2}}}+\frac{e^{\mathrm{i}\left(c_{\mathrm{p}} \omega_{1}|x-y|-c_{\mathrm{s}} \omega_{2}|x-z|\right)}}{c_{\mathrm{p}}^{j_{1}-\frac{3}{2}} c_{\mathrm{S}}^{j_{2}-\frac{1}{2}}}\right] \frac{\left(x_{2}-y_{2}\right)^{2} \mathbb{E}\left(f_{2}(y) f_{2}(z)\right)}{|x-y|^{j_{1}+\frac{5}{2}}|x-z|^{j_{2}+\frac{3}{2}}} \mathrm{~d} y \mathrm{~d} z, \\
& \mathbb{E}\left(\tilde{u}_{23}\left(x, \omega_{1}\right) \overline{\tilde{u}_{23}\left(x, \omega_{2}\right)}\right)=\frac{1}{16} \sum_{j_{1}, j_{2}=0}^{4} \frac{a_{j_{1}}^{(2)} \overline{a_{j_{2}}^{(2)}}}{\omega_{1}^{j_{1}+\frac{1}{2}} \omega_{2}^{j_{2}+\frac{1}{2}}} \\
& \times \int_{\mathbb{R}^{4}}\left[\frac{e^{\mathrm{i}\left(c_{\mathrm{s}} \omega_{1}|x-y|-c_{\mathrm{s}} \omega_{2}|x-z|\right)}}{c_{\mathrm{s}}^{j_{1}+j_{2}-3}}+\frac{e^{\mathrm{i}\left(c_{\mathrm{p}} \omega_{1}|x-y|-c_{\mathrm{p}} \omega_{2}|x-z|\right)}}{c_{\mathrm{p}}^{j_{1}+j_{2}-3}}\right. \\
& \left.-\frac{e^{\mathrm{i}\left(c_{\mathrm{s}} \omega_{1}|x-y|-c_{\mathrm{p}} \omega_{2}|x-z|\right)}}{c_{\mathrm{s}}^{j_{1}-\frac{3}{2}} c_{\mathrm{p}}^{j_{2}-\frac{3}{2}}}-\frac{e^{\mathrm{i}\left(c_{\mathrm{p}} \omega_{1}|x-y|-c_{\mathrm{s}} \omega_{2}|x-z|\right)}}{c_{\mathrm{p}}^{j_{1}-\frac{3}{2}} c_{\mathrm{s}}^{j_{2}-\frac{3}{2}}}\right] \frac{\left(x_{2}-y_{2}\right)^{2}\left(x_{2}-z_{2}\right)^{2} \mathbb{E}\left(f_{2}(y) f_{2}(z)\right)}{|x-y|^{j_{1}+\frac{5}{2}}|x-z|^{j_{2}+\frac{5}{2}}} \mathrm{~d} y \mathrm{~d} z, \\
& \mathbb{E}\left(\tilde{u}_{24}\left(x, \omega_{1}\right) \overline{\tilde{u}_{24}\left(x, \omega_{2}\right)}\right)=\frac{1}{16} \sum_{j_{1}, j_{2}=0}^{4} \frac{a_{j_{1}}^{(2)} \overline{a_{j_{2}}^{(2)}}}{\omega_{1}^{j_{1}+\frac{1}{2}} \omega_{2}^{j_{2}+\frac{1}{2}}} \\
& \times \int_{\mathbb{R}^{4}}\left[\frac{e^{\mathrm{i}\left(c_{\mathrm{s}} \omega_{1}|x-y|-c_{\mathrm{s}} \omega_{2}|x-z|\right)}}{c_{\mathrm{S}}^{j_{1}+j_{2}-3}}+\frac{e^{\mathrm{i}\left(c_{\mathrm{p}} \omega_{1}|x-y|-c_{\mathrm{p}} \omega_{2}|x-z|\right)}}{c_{\mathrm{p}}^{j_{1}+j_{2}-3}}-\frac{e^{\mathrm{i}\left(c_{\mathrm{s}} \omega_{1}|x-y|-c_{\mathrm{p}} \omega_{2}|x-z|\right)}}{c_{\mathrm{S}}^{j_{1}-\frac{3}{2}} c_{\mathrm{p}}^{j_{2}-\frac{3}{2}}}\right. \\
& \left.-\frac{e^{\mathrm{i}\left(c_{\mathrm{p}} \omega_{1}|x-y|-c_{\mathrm{s}} \omega_{2}|x-z|\right)}}{c_{\mathrm{p}}^{j_{1}-\frac{3}{2}} c_{\mathrm{s}}^{j_{2}-\frac{3}{2}}}\right] \frac{\left(x_{1}-y_{1}\right)\left(x_{2}-y_{2}\right)\left(x_{1}-z_{1}\right)\left(x_{2}-z_{2}\right)}{|x-y|^{j_{1}+\frac{5}{2}}|x-z|^{j_{2}+\frac{5}{2}}} \mathbb{E}\left(f_{1}(y) f_{1}(z)\right) \mathrm{d} y \mathrm{~d} z .
\end{aligned}
$$

A direct application of Lemma 3.5 to each item on the right hand side of (4.8) gives the following lemma.

Lemma 4.5. For $\omega_{1} \geq 1, \omega_{2} \geq 1$, the following estimates

$$
\begin{aligned}
& \left|\mathbb{E}\left(\tilde{\boldsymbol{u}}\left(x, \omega_{1}\right) \cdot \tilde{\boldsymbol{u}}\left(x, \omega_{2}\right)\right)\right| \leq c_{n}\left(1+\left|\omega_{1}-\omega_{2}\right|\right)^{-n}\left(\omega_{1}+\omega_{2}\right)^{-m-1}, \\
& \left|\mathbb{E}\left(\tilde{\boldsymbol{u}}\left(x, \omega_{1}\right) \cdot \tilde{\boldsymbol{u}}\left(x, \omega_{2}\right)\right)\right| \leq c_{n}\left(\omega_{1}+\omega_{2}\right)^{-n}\left(1+\left|\omega_{1}-\omega_{2}\right|\right)^{-m}
\end{aligned}
$$

holds uniformly for $x \in U$, where $n \in \mathbb{N}$ is arbitrary and $c_{n}>0$ is a constant depending only on $n$.

To obtain the relation between the scattering data and the function in the principle symbol, it is required to estimate the order of $\mathbb{E}(\tilde{u}(x, \omega) \cdot \overline{\tilde{u}(x, \omega)})$. According to (4.8) where we set $\omega_{1}=\omega_{2}=\omega$, it reduces to estimate the order of $\mathbb{E}\left(\tilde{u}_{i j_{1}}(x, \omega) \overline{\tilde{u}_{i j_{2}}(x, \omega)}\right)$ for $i=1,2, j_{1}, j_{2}=1,2,3,4$. Applying Lemma 3.7 gives that

$$
\begin{array}{ll}
\mathbb{E}\left(\tilde{u}_{11}(x, \omega) \overline{\tilde{u}_{12}(x, \omega)}\right)=O\left(\omega^{-(m+2)}\right), & \mathbb{E}\left(\tilde{u}_{12}(x, \omega) \overline{\tilde{u}_{11}(x, \omega)}\right)=O\left(\omega^{-(m+2)}\right), \\
\mathbb{E}\left(\tilde{u}_{12}(x, \omega) \overline{\tilde{u}_{12}(x, \omega)}\right)=O\left(\omega^{-(m+2)}\right), & \mathbb{E}\left(\tilde{u}_{12}(x, \omega) \overline{\tilde{u}_{13}(x, \omega)}\right)=O\left(\omega^{-(m+2)}\right), \\
\mathbb{E}\left(\tilde{u}_{13}(x, \omega) \overline{\tilde{u}_{12}(x, \omega)}\right)=O\left(\omega^{-(m+2)}\right), & \mathbb{E}\left(\tilde{u}_{21}(x, \omega) \overline{\tilde{u}_{22}(x, \omega)}\right)=O\left(\omega^{-(m+2)}\right), \\
\mathbb{E}\left(\tilde{u}_{22}(x, \omega) \overline{\tilde{u}_{21}(x, \omega)}\right)=O\left(\omega^{-(m+2)}\right), & \mathbb{E}\left(\tilde{u}_{22}(x, \omega) \overline{\tilde{u}_{22}(x, \omega)}\right)=O\left(\omega^{-(m+2)}\right), \\
\mathbb{E}\left(\tilde{u}_{22}(x, \omega) \overline{\tilde{u}_{23}(x, \omega)}\right)=O\left(\omega^{-(m+2)}\right), & \mathbb{E}\left(\tilde{u}_{23}(x, \omega) \overline{\tilde{u}_{22}(x, \omega)}\right)=O\left(\omega^{-(m+2)}\right),
\end{array}
$$


and

$$
\begin{aligned}
& \mathbb{E}\left(\tilde{u}_{11}(x, \omega) \overline{\tilde{u}_{11}(x, \omega)}\right)=N_{1}^{(2)}(x) \omega^{-(m+1)}+O\left(\omega^{-(m+2)}\right) \\
& \mathbb{E}\left(\tilde{u}_{11}(x, \omega) \overline{\tilde{u}_{13}(x, \omega)}\right)=N_{2}^{(2)}(x, \omega) \omega^{-(m+1)}+O\left(\omega^{-(m+2)}\right) \\
& \mathbb{E}\left(\tilde{u}_{13}(x, \omega) \overline{\tilde{u}_{11}(x, \omega)}\right)=N_{3}^{(2)}(x, \omega) \omega^{-(m+1)}+O\left(\omega^{-(m+2)}\right) \\
& \mathbb{E}\left(\tilde{u}_{13}(x, \omega) \overline{\tilde{u}_{13}(x, \omega)}\right)=N_{4}^{(2)}(x, \omega) \omega^{-(m+1)}+O\left(\omega^{-(m+2)}\right) \\
& \mathbb{E}\left(\tilde{u}_{14}(x, \omega) \overline{\tilde{u}_{14}(x, \omega)}\right)=N_{5}^{(2)}(x, \omega) \omega^{-(m+1)}+O\left(\omega^{-(m+2)}\right) \\
& \mathbb{E}\left(\tilde{u}_{21}(x, \omega) \overline{\tilde{u}_{21}(x, \omega)}\right)=N_{6}^{(2)}(x) \omega^{-(m+1)}+O\left(\omega^{-(m+2)}\right) \\
& \mathbb{E}\left(\tilde{u}_{21}(x, \omega) \overline{\tilde{u}_{23}(x, \omega)}\right)=N_{7}^{(2)}(x, \omega) \omega^{-(m+1)}+O\left(\omega^{-(m+2)}\right) \\
& \mathbb{E}\left(\tilde{u}_{23}(x, \omega) \overline{\tilde{u}_{21}(x, \omega)}\right)=N_{8}^{(2)}(x, \omega) \omega^{-(m+1)}+O\left(\omega^{-(m+2)}\right) \\
& \mathbb{E}\left(\tilde{u}_{23}(x, \omega) \overline{\tilde{u}_{23}(x, \omega)}\right)=N_{9}^{(2)}(x, \omega) \omega^{-(m+1)}+O\left(\omega^{-(m+2)}\right) \\
& \mathbb{E}\left(\tilde{u}_{24}(x, \omega) \overline{\tilde{u}_{24}(x, \omega)}\right)=N_{10}^{(2)}(x, \omega) \omega^{-(m+1)}+O\left(\omega^{-(m+2)}\right),
\end{aligned}
$$

where

$$
\begin{aligned}
N_{1}^{(2)}(x) & =N_{6}^{(2)}(x)=a_{1} \int_{\mathbb{R}^{2}} \frac{1}{|x-y|} \phi(y) \mathrm{d} y \\
N_{2}^{(2)}(x, \omega) & =\int_{\mathbb{R}^{2}}\left(a_{2} e^{\mathrm{i}\left(c_{\mathrm{s}}-c_{\mathrm{p}}\right)|x-y| \omega}-a_{1}\right) \frac{\left(x_{1}-y_{1}\right)^{2}}{|x-y|^{3}} \phi(y) \mathrm{d} y, \\
N_{3}^{(2)}(x, \omega) & =\int_{\mathbb{R}^{2}}\left(a_{2} e^{\mathrm{i}\left(c_{\mathrm{p}}-c_{\mathrm{s}}\right)|x-y| \omega}-a_{1}\right) \frac{\left(x_{1}-y_{1}\right)^{2}}{|x-y|^{3}} \phi(y) \mathrm{d} y, \\
N_{4}^{(2)}(x, \omega) & =\int_{\mathbb{R}^{2}}\left(a_{3}-2 a_{2} \cos \left(\left(c_{\mathrm{s}}-c_{\mathrm{p}}\right)|x-y| \omega\right)\right) \frac{\left(x_{1}-y\right)^{4}}{|x-y|^{5}} \phi(y) \mathrm{d} y, \\
N_{5}^{(2)}(x, \omega) & =N_{10}^{(2)}(x, \omega)=\int_{\mathbb{R}^{2}}\left(a_{3}-2 a_{2} \cos \left(\left(c_{\mathrm{s}}-c_{\mathrm{p}}\right)|x-y| \omega\right)\right) \frac{\left(x_{1}-y_{1}\right)^{2}\left(x_{2}-y_{2}\right)^{2}}{|x-y|^{5}} \phi(y) \mathrm{d} y, \\
N_{7}^{(2)}(x, \omega) & =\int_{\mathbb{R}^{2}}\left(a_{2} e^{\mathrm{i}\left(c_{\mathrm{s}}-c_{\mathrm{p}}\right)|x-y| \omega}-a_{1}\right) \frac{\left(x_{2}-y_{2}\right)^{2}}{|x-y|^{3}} \phi(y) \mathrm{d} y, \\
N_{8}^{(2)}(x, \omega) & =\int_{\mathbb{R}^{2}}\left(a_{2} e^{\mathrm{i}\left(c_{\mathrm{p}}-c_{\mathrm{s}}\right)|x-y| \omega}-a_{1}\right) \frac{\left(x_{2}-y_{2}\right)^{2}}{|x-y|^{3}} \phi(y) \mathrm{d} y, \\
N_{9}^{(2)}(x, \omega) & =\int_{\mathbb{R}^{2}}\left(a_{3}-2 a_{2} \cos \left(\left(c_{\mathrm{s}}-c_{\mathrm{p}}\right)|x-y| \omega\right)\right) \frac{\left(x_{2}-y\right)^{4}}{|x-y|^{5}} \phi(y) \mathrm{d} y .
\end{aligned}
$$

Here, $a_{1}, a_{2}$, and $a_{3}$ are positive constants given by

$$
a_{1}=\frac{1}{32 \pi c_{\mathrm{s}}^{m-3}}, \quad a_{2}=\frac{\left(c_{\mathrm{s}} c_{\mathrm{p}}\right)^{\frac{3}{2}}}{32 \pi}\left(\frac{2}{c_{\mathrm{s}}+c_{\mathrm{p}}}\right)^{m}, \quad a_{3}=\frac{1}{32 \pi}\left(\frac{1}{c_{\mathrm{s}}^{m-3}}+\frac{1}{c_{\mathrm{p}}^{m-3}}\right) .
$$

By (4.8) and a simple calculation, we obtain

$$
\mathbb{E}(\tilde{\boldsymbol{u}}(x, \omega) \cdot \overline{\tilde{\boldsymbol{u}}(x, \omega)})=T_{\mathrm{E}}^{(2)}(x) \omega^{-(m+1)}+O\left(\omega^{-(m+2)}\right),
$$

where

$$
T_{\mathrm{E}}^{(2)}(x)=\sum_{j=1}^{10} N_{j}^{(2)}(x, \omega)=a_{3} \int_{\mathbb{R}^{2}} \frac{1}{|x-y|} \phi(y) d y .
$$

Now we are ready to present the main result for elastic waves in the two dimensions. 
Theorem 4.6. Let the external source $\boldsymbol{f}$ be a microlocally isotropic Gaussian random vector field which satisfies Assumption $C$. Then for all $x \in U$, it holds almost surely that

$$
\lim _{Q \rightarrow \infty} \frac{1}{Q-1} \int_{1}^{Q} \omega^{m+1}|\boldsymbol{u}(x, \omega)|^{2} \mathrm{~d} \omega=T_{\mathrm{E}}^{(2)}(x),
$$

where $T_{\mathrm{E}}^{(2)}(x)$ is given in (4.10). Moreover, the scattering data $T_{\mathrm{E}}^{(2)}(x)$, for $x \in U$ uniquely determine the micro-correlation strength $\phi$ through the linear integral equation 4.10).

Proof. Since

$$
\begin{aligned}
& \frac{1}{Q-1} \int_{1}^{Q} \omega^{m+1}|\boldsymbol{u}(x, \omega)|^{2} \mathrm{~d} \omega \\
& =\frac{1}{Q-1} \int_{1}^{Q} \omega^{m+1}|\tilde{\boldsymbol{u}}(x, \omega)+\boldsymbol{u}(x, \omega)-\tilde{\boldsymbol{u}}(x, \omega)|^{2} \mathrm{~d} \omega \\
& =\frac{1}{Q-1} \int_{1}^{Q} \omega^{m+1}|\tilde{\boldsymbol{u}}(x, \omega)|^{2} d \omega+\frac{1}{Q-1} \int_{1}^{Q} \omega^{m+1}|\boldsymbol{u}(x, \omega)-\tilde{\boldsymbol{u}}(x, \omega)|^{2} \mathrm{~d} \omega \\
& \quad+\frac{2}{Q-1} \int_{1}^{Q} \omega^{m+1} \Re[\overline{\tilde{\boldsymbol{u}}(x, \omega)}(\boldsymbol{u}(x, \omega)-\tilde{\boldsymbol{u}}(x, \omega))] \mathrm{d} \omega
\end{aligned}
$$

thus, (4.11) holds as long as we show that

$$
\begin{aligned}
& \lim _{Q \rightarrow \infty} \frac{1}{Q-1} \int_{1}^{Q} \omega^{m+1}|\tilde{\boldsymbol{u}}(x, \omega)|^{2} \mathrm{~d} \omega=T_{\mathrm{E}}^{(2)}(x), \\
& \lim _{Q \rightarrow \infty} \frac{1}{Q-1} \int_{1}^{Q} \omega^{m+1}|\boldsymbol{u}(x, \omega)-\tilde{\boldsymbol{u}}(x, \omega)|^{2} \mathrm{~d} \omega=0, \\
& \lim _{Q \rightarrow \infty} \frac{2}{Q-1} \int_{1}^{Q} \omega^{m+1} \Re[\overline{\tilde{\boldsymbol{u}}(x, \omega)}(\boldsymbol{u}(x, \omega)-\tilde{\boldsymbol{u}}(x, \omega))] \mathrm{d} \omega=0 .
\end{aligned}
$$

To prove (4.12), we denote $Y(x, \omega)=\omega^{m+1}\left(|\tilde{\boldsymbol{u}}(x, \omega)|^{2}-\mathbb{E}\left(|\tilde{\boldsymbol{u}}(x, \omega)|^{2}\right)\right)$, which yields

$$
\int_{1}^{Q} \omega^{m+1}|\tilde{\boldsymbol{u}}(x, \omega)|^{2} \mathrm{~d} \omega=\int_{1}^{Q} \omega^{m+1} \mathbb{E}\left(|\tilde{\boldsymbol{u}}(x, \omega)|^{2}\right) \mathrm{d} \omega+\int_{1}^{Q} Y(x, \omega) \mathrm{d} \omega .
$$

Hence, (4.12) holds as long as we prove

$$
\lim _{Q \rightarrow \infty} \frac{1}{Q-1} \int_{1}^{Q} \omega^{m+1} \mathbb{E}\left(|\tilde{\boldsymbol{u}}(x, \omega)|^{2}\right) \mathrm{d} \omega=T_{\mathrm{E}}^{(2)}(x), \quad \lim _{Q \rightarrow \infty} \frac{1}{Q-1} \int_{1}^{Q} Y(x, \omega) \mathrm{d} \omega=0 .
$$

Multiplying (4.9) by $\omega^{m+1}$ and integrating with respect to the frequency $\omega$ in the internal $(1, Q)$, we arrive at

$$
\frac{1}{Q-1} \int_{1}^{Q} \omega^{m+1} \mathbb{E}\left(|\tilde{\boldsymbol{u}}(x, \omega)|^{2}\right) \mathrm{d} \omega=\frac{1}{Q-1} \int_{1}^{Q}\left(T_{\mathrm{E}}^{(2)}(x)+O\left(\omega^{m-3}\right)\right) \mathrm{d} \omega .
$$

It is clear to note that

$$
\left|\frac{1}{Q-1} \int_{1}^{Q} O\left(\omega^{m-3}\right) \mathrm{d} \omega\right| \lesssim \frac{1}{Q-1} \int_{1}^{Q} \omega^{m-3} \mathrm{~d} \omega \rightarrow 0 \quad \text { as } Q \rightarrow \infty,
$$

where we use the fact that $m \in\left[d, d+\frac{1}{2}\right.$ ). Thus, the first equation in (4.15) holds. Now we focus on the second equation in (4.15) and want to show that

$$
\lim _{Q \rightarrow \infty} \frac{1}{Q-1} \int_{1}^{Q} Y(x, \omega) \mathrm{d} \omega=0 .
$$


By the definition of $Y(x, \omega)$,

$$
\begin{aligned}
Y(x, \omega) & =\omega^{m+1}\left(|\tilde{\boldsymbol{u}}(x, \omega)|^{2}-\mathbb{E}\left(|\tilde{\boldsymbol{u}}(x, \omega)|^{2}\right)\right) \\
& =\omega^{m+1}\left((\Re \tilde{\boldsymbol{u}}(x, \omega))^{2}-\mathbb{E}(\Re \tilde{\boldsymbol{u}}(x, \omega))^{2}+(\Im \tilde{\boldsymbol{u}}(x, \omega))^{2}-\mathbb{E}(\Im \tilde{\boldsymbol{u}}(x, \omega))^{2}\right) .
\end{aligned}
$$

Therefore,

$$
\mathbb{E}\left(Y\left(x, \omega_{1}\right) Y\left(x, \omega_{2}\right)\right)=I_{E, 1}+I_{E, 2}+I_{E, 3}+I_{E, 4},
$$

where

$$
\begin{aligned}
& I_{E, 1}=\omega_{1}^{m+1} \omega_{2}^{m+1} \mathbb{E}\left[\left(\left(\Re \tilde{\boldsymbol{u}}\left(x, \omega_{1}\right)\right)^{2}-\mathbb{E}\left(\Re \tilde{\boldsymbol{u}}\left(x, \omega_{1}\right)\right)^{2}\right)\left(\left(\Re \tilde{\boldsymbol{u}}\left(x, \omega_{2}\right)\right)^{2}-\mathbb{E}\left(\Re \tilde{\boldsymbol{u}}\left(x, \omega_{2}\right)\right)^{2}\right)\right], \\
& I_{E, 2}=\omega_{1}^{m+1} \omega_{2}^{m+1} \mathbb{E}\left[\left(\left(\Re \tilde{\boldsymbol{u}}\left(x, \omega_{1}\right)\right)^{2}-\mathbb{E}\left(\Re \tilde{\boldsymbol{u}}\left(x, \omega_{1}\right)\right)^{2}\right)\left(\left(\Im \tilde{\boldsymbol{u}}\left(x, \omega_{2}\right)\right)^{2}-\mathbb{E}\left(\Im \tilde{\boldsymbol{u}}\left(x, \omega_{2}\right)\right)^{2}\right)\right], \\
& I_{E, 3}=\omega_{1}^{m+1} \omega_{2}^{m+1} \mathbb{E}\left[\left(\left(\Im \tilde{\boldsymbol{u}}\left(x, \omega_{1}\right)\right)^{2}-\mathbb{E}\left(\Im \tilde{\boldsymbol{u}}\left(x, \omega_{1}\right)\right)^{2}\right)\left(\left(\Re \tilde{\boldsymbol{u}}\left(x, \omega_{2}\right)\right)^{2}-\mathbb{E}\left(\Re \tilde{\boldsymbol{u}}\left(x, \omega_{2}\right)\right)^{2}\right)\right], \\
& I_{E, 4}=\omega_{1}^{m+1} \omega_{2}^{m+1} \mathbb{E}\left[\left(\left(\Im \tilde{\boldsymbol{u}}\left(x, \omega_{1}\right)\right)^{2}-\mathbb{E}\left(\Im \tilde{\boldsymbol{u}}\left(x, \omega_{1}\right)\right)^{2}\right)\left(\left(\Im \tilde{\boldsymbol{u}}\left(x, \omega_{2}\right)\right)^{2}-\mathbb{E}\left(\Im \tilde{\boldsymbol{u}}\left(x, \omega_{2}\right)\right)^{2}\right)\right] .
\end{aligned}
$$

Combing the expression of $\tilde{\boldsymbol{u}}(x, \omega)$ and the assumption $\mathbb{E}\left(f_{1}\right)=0, \mathbb{E}\left(f_{2}\right)=0$ gives that $\Re \tilde{\boldsymbol{u}}(x, \omega)$ and $\Im \tilde{\boldsymbol{u}}(x, \omega)$ are zero-mean Gaussian random variables. Applying Lemmas 2.3 and 4.5 leads to

$$
\begin{aligned}
I_{E, 1} & =2 \omega_{1}^{m+1} \omega_{2}^{m+1}\left[\mathbb{E}\left(\Re \tilde{\boldsymbol{u}}\left(x, \omega_{1}\right) \Re \tilde{\boldsymbol{u}}\left(x, \omega_{2}\right)\right)\right]^{2} \\
& =\frac{1}{2} \omega_{1}^{m+1} \omega_{2}^{m+1}\left[\mathbb{E}\left(\Re\left(\tilde{\boldsymbol{u}}\left(x, \omega_{1}\right) \tilde{\boldsymbol{u}}\left(x, \omega_{2}\right)\right)+\Re\left(\tilde{\boldsymbol{u}}\left(x, \omega_{1}\right) \overline{\tilde{\boldsymbol{u}}\left(x, \omega_{2}\right)}\right)\right)\right]^{2} \\
& \lesssim\left[\frac{\omega_{1}^{\frac{m+1}{2}} \omega_{2}^{\frac{m+1}{2}}}{\left(\omega_{1}+\omega_{2}\right)^{n}\left(1+\left|\omega_{1}-\omega_{2}\right|\right)^{m}}+\frac{\omega_{1}^{\frac{m+1}{2}} \omega_{2}^{\frac{m+1}{2}}}{\left(\omega_{1}+\omega_{2}\right)^{m+1}\left(1+\left|\omega_{1}-\omega_{2}\right|\right)^{n}}\right]^{2} \\
& \lesssim\left[\frac{1}{\left(1+\left|\omega_{1}-\omega_{2}\right|\right)^{m}}+\frac{1}{\left(1+\left|\omega_{1}-\omega_{2}\right|\right)^{n}}\right]^{2} .
\end{aligned}
$$

We can obtain the same estimates for $I_{E, 2}, I_{E, 3}$, and $I_{E, 4}$ by the similar arguments. Thus, an application of Lemma 2.4 gives

$$
\lim _{Q \rightarrow \infty} \frac{1}{Q-1} \int_{1}^{Q} Y(x, \omega) \mathrm{d} \omega=0
$$

To prove (4.13), from lemma 4.4, we obtain

$$
\begin{aligned}
& \left|\frac{1}{Q-1} \int_{1}^{Q} \omega^{m+1}\right| \boldsymbol{u}(x, \omega)-\left.\tilde{\boldsymbol{u}}(x, \omega)\right|^{2} \mathrm{~d} \omega \mid \lesssim \frac{1}{Q-1} \int_{1}^{Q} \omega^{m+1} \omega^{-7} \mathrm{~d} \omega \\
& \lesssim \frac{1}{Q-1} \int_{1}^{Q} \omega^{m-6} \mathrm{~d} \omega \lesssim \frac{1}{m-5} \frac{Q^{m-5}-1}{Q-1} \rightarrow 0 \quad \text { as } Q \rightarrow \infty
\end{aligned}
$$


To prove (4.14), by the Hölder inequality, we have

$$
\begin{aligned}
& \left|\frac{2}{Q-1} \int_{1}^{Q} \omega^{m+1} \Re[\overline{\tilde{\boldsymbol{u}}(x, \omega)}(\boldsymbol{u}(x, \omega)-\tilde{\boldsymbol{u}}(x, \omega))] \mathrm{d} \omega\right| \\
& \lesssim \frac{2}{Q-1} \int_{1}^{Q} \omega^{m+1}|\tilde{\boldsymbol{u}}(x, \omega)||\boldsymbol{u}(x, \omega)-\tilde{\boldsymbol{u}}(x, \omega)| \mathrm{d} \omega \\
& \lesssim 2\left[\frac{1}{Q-1} \int_{1}^{Q} \omega^{m+1}|\tilde{\boldsymbol{u}}(x, \omega)|^{2} \mathrm{~d} \omega\right]^{\frac{1}{2}}\left[\frac{1}{Q-1} \int_{1}^{Q} \omega^{m+1}|\boldsymbol{u}(x, \omega)-\tilde{\boldsymbol{u}}(x, \omega)|^{2} \mathrm{~d} \omega\right]^{\frac{1}{2}} \\
& \rightarrow 2 T_{\mathrm{E}}^{(2)}(x)^{\frac{1}{2}} \cdot 0=0 \quad \text { as } Q \rightarrow \infty
\end{aligned}
$$

The unique determination of $\phi$ by $T_{\mathrm{E}}^{(2)}(x)$ for $x \in U$ is a direct consequence of Lemma 3.8.

4.3. The three-dimensional case. To derive the linear relation between the scattering data and the function in the principle symbol, it is required to express the wave field more explicitly than (4.7). Substituting (4.6) into (4.3) gives the wave field $\boldsymbol{u}(x, \omega)=\left(u_{1}(x, \omega), u_{2}(x, \omega), u_{3}(x, \omega)\right)^{\top}$ where each component $u_{i}(x, \omega)$ is given by

$$
u_{i}(x, \omega)=u_{i 1}(x, \omega)+u_{i 2}(x, \omega)+u_{i 3}(x, \omega) .
$$

Here

$$
\begin{aligned}
u_{i 1}(x, \omega) & =\frac{1}{4 \pi \mu} \int_{\mathbb{R}^{3}} \frac{e^{\mathrm{i} \kappa_{s}|x-y|}}{|x-y|} f_{i}(y) \mathrm{d} y \\
u_{i 2}(x, \omega)= & \frac{1}{4 \pi \omega^{2}} \int_{\mathbb{R}^{3}} \frac{1}{|x-y|^{3}}\left[e^{\mathrm{i} \kappa_{s}|x-y|}\left(\mathrm{i} \kappa_{s}|x-y|-1\right)-e^{\mathrm{i} \kappa_{p}|x-y|}\left(\mathrm{i} \kappa_{p}|x-y|-1\right)\right] f_{i}(y) \mathrm{d} y, \\
u_{i 3}(x, \omega)=-\frac{1}{4 \pi \omega^{2}} \int_{\mathbb{R}^{3}}\left[\left(\frac{3\left(\mathrm{i} \kappa_{s}|x-y|-1\right)}{|x-y|^{2}}+\kappa_{s}^{2}\right) e^{\mathrm{i} \kappa_{s}|x-y|}\right. & \left.-\left(\frac{3\left(\mathrm{i} \kappa_{p}|x-y|-1\right)}{|x-y|^{2}}+\kappa_{p}^{2}\right) e^{\mathrm{i} \kappa_{p}|x-y|}\right] \frac{\left(x_{i}-y_{i}\right)}{|x-y|^{3}}(x-y) \cdot \boldsymbol{f}(y) \mathrm{d} y .
\end{aligned}
$$

As mentioned above, we need to derive the relationship between the scattering data and the function $\phi$ in the principle symbol. For this end, it is required to calculate the exception $\mathbb{E}\left(\boldsymbol{u}\left(x, \omega_{1}\right)\right.$. $\left.\overline{\boldsymbol{u}\left(x, \omega_{2}\right)}\right)$. Noting that $\boldsymbol{u}(x, \omega) \in \mathbb{C}^{3}$ and each component has been decomposed into three parts, we obtain

$$
\begin{aligned}
& \mathbb{E}(\boldsymbol{u}(x, \omega) \cdot \overline{\boldsymbol{u}(x, \omega)}) \\
& =\mathbb{E}\left(u_{1}(x, \omega) \overline{u_{1}(x, \omega)}+u_{2}(x, \omega) \overline{u_{2}(x, \omega)}+u_{3}(x, \omega) \overline{u_{3}(x, \omega)}\right) \\
& =\sum_{i, j=1}^{3} \mathbb{E}\left(u_{1 i}(x, \omega) \overline{u_{1 j}(x, \omega)}+u_{2 i}(x, \omega) \overline{u_{2 j}(x, \omega)}+u_{3 i}(x, \omega) \overline{u_{3 j}(x, \omega)}\right) .
\end{aligned}
$$

To calculate the expectation $\mathbb{E}\left(\boldsymbol{u}\left(x, \omega_{1}\right) \cdot \overline{\boldsymbol{u}\left(x, \omega_{2}\right)}\right)$, it is required to calculate the items on the right hand side of (4.16). Using the expression of $u_{i j}(x, \omega)(i, j=1,2,3)$, we have from direct calculations that

$$
\begin{array}{r}
\mathbb{E}\left(u_{i 1}\left(x, \omega_{1}\right) \overline{u_{i 1}\left(x, \omega_{2}\right)}\right)=\frac{1}{16 \pi^{2} \mu^{2}} \int_{\mathbb{R}^{6}} \frac{e^{\mathrm{i}\left(c_{\mathrm{s}} \omega_{1}|x-y|-c_{\mathrm{s}} \omega_{2}|x-z|\right)}}{|x-y||x-z|} \mathbb{E}\left(f_{i}(y) f_{i}(z)\right) \mathrm{d} y \mathrm{~d} z, \\
\mathbb{E}\left(u_{i 1}\left(x, \omega_{1}\right) \overline{u_{i 2}\left(x, \omega_{2}\right)}\right)=-\frac{1}{16 \pi^{2} \mu \omega_{2}^{2}} \int_{\mathbb{R}^{6}}\left[e^{\mathrm{i}\left(c_{\mathrm{s}} \omega_{1}|x-y|-c_{\mathrm{s}} \omega_{2}|x-z|\right)}\left(\mathrm{i} c_{\mathrm{s}} \omega_{2}|x-z|+1\right)\right. \\
\left.-e^{\mathrm{i}\left(c_{\mathrm{s}} \omega_{1}|x-y|-c_{\mathrm{p}} \omega_{2}|x-z|\right)}\left(\mathrm{i} c_{\mathrm{p}} \omega_{2}|x-z|+1\right)\right] \frac{\mathbb{E}\left(f_{i}(y) f_{i}(z)\right)}{|x-y||x-z|^{3}} \mathrm{~d} y \mathrm{~d} z,
\end{array}
$$




$$
\begin{aligned}
& \mathbb{E}\left(u_{i 1}\left(x, \omega_{1}\right) \overline{u_{i 3}\left(x, \omega_{2}\right)}\right)=\frac{1}{16 \pi^{2} \mu \omega_{2}^{2}} \int_{\mathbb{R}^{6}}\left[\left(\frac{3}{|x-z|^{2}}\left(\mathrm{i} c_{\mathrm{s}} \omega_{2}|x-z|+1\right)-c_{\mathrm{s}}^{2} \omega_{2}^{2}\right) \times\right. \\
& \left.e^{\mathrm{i}\left(c_{\mathrm{s}} \omega_{1}|x-y|-c_{\mathrm{s}} \omega_{2}|x-z|\right)}-\left(\frac{3}{|x-z|^{2}}\left(\mathrm{i} c_{\mathrm{p}} \omega_{2}|x-z|+1\right)-c_{\mathrm{p}}^{2} \omega_{2}^{2}\right) e^{\mathrm{i}\left(c_{\mathrm{s}} \omega_{1}|x-y|-c_{\mathrm{p}} \omega_{2}|x-z|\right)}\right] \\
& \times \frac{\left(x_{i}-z_{i}\right)^{2}}{|x-y||x-z|^{3}} \mathbb{E}\left(f_{i}(y) f_{i}(z)\right) \mathrm{d} y \mathrm{~d} z, \\
& \mathbb{E}\left(u_{i 2}\left(x, \omega_{1}\right) \overline{u_{i 1}\left(x, \omega_{2}\right)}\right)=\frac{1}{16 \pi^{2} \mu \omega_{1}^{2}} \int_{\mathbb{R}^{6}}\left[\left(\mathrm{i} c_{\mathrm{s}} \omega_{1}|x-y|-1\right) e^{\mathrm{i}\left(c_{\mathrm{s}} \omega_{1}|x-y|-c_{\mathrm{s}} \omega_{2}|x-z|\right)}\right. \\
& \left.-\left(\mathrm{i} c_{\mathrm{p}} \omega_{1}|x-y|-1\right) e^{\mathrm{i}\left(c_{\mathrm{p}} \omega_{1}|x-y|-c_{\mathrm{s}} \omega_{2}|x-z|\right)}\right] \frac{\mathbb{E}\left(f_{i}(y) f_{i}(z)\right)}{|x-y|^{3}|x-z|} \mathrm{d} y \mathrm{~d} z, \\
& \mathbb{E}\left(u_{i 2}\left(x, \omega_{1}\right) \overline{u_{i 2}\left(x, \omega_{2}\right)}\right)=\frac{1}{16 \pi^{2} \omega_{1}^{2} \omega_{2}^{2}} \times \\
& \int_{\mathbb{R}^{6}}\left[\left(\mathrm{i} c_{\mathrm{S}} \omega_{1}|x-y|-1\right)\left(-\mathrm{i} c_{\mathrm{S}} \omega_{2}|x-z|-1\right) e^{\mathrm{i}\left(c_{\mathrm{s}} \omega_{1}|x-y|-c_{\mathrm{s}} \omega_{2}|x-z|\right)}\right. \\
& +\left(\mathrm{i} c_{\mathrm{p}} \omega_{1}|x-y|-1\right)\left(-\mathrm{i} c_{\mathrm{p}} \omega_{2}|x-z|-1\right) e^{\mathrm{i}\left(c_{\mathrm{p}} \omega_{1}|x-y|-c_{\mathrm{p}} \omega_{2}|x-z|\right)} \\
& -\left(\mathrm{i} c_{\mathrm{s}} \omega_{1}|x-y|-1\right)\left(-\mathrm{i} c_{\mathrm{p}} \omega_{2}|x-z|-1\right) e^{\mathrm{i}\left(c_{\mathrm{s}} \omega_{1}|x-y|-c_{\mathrm{p}} \omega_{2}|x-z|\right)} \\
& \left.-\left(\mathrm{i} c_{\mathrm{p}} \omega_{1}|x-y|-1\right)\left(-\mathrm{i} c_{\mathrm{s}} \omega_{2}|x-z|-1\right) e^{\mathrm{i}\left(c_{\mathrm{p}} \omega_{1}|x-y|-c_{\mathrm{s}} \omega_{2}|x-z|\right)}\right] \frac{\mathbb{E}\left(f_{i}(y) f_{i}(z)\right)}{|x-y|^{3}|x-z|^{3}} \mathrm{~d} y \mathrm{~d} z, \\
& \mathbb{E}\left(u_{i 2}\left(x, \omega_{1}\right) \overline{u_{i 3}\left(x, \omega_{2}\right)}\right)=\frac{1}{16 \pi^{2} \omega_{1}^{2} \omega_{2}^{2}} \times \\
& \int_{\mathbb{R}^{6}}\left[\left(\mathrm{i} c_{\mathrm{S}} \omega_{1}|x-y|-1\right)\left(\frac{3}{|x-z|^{2}}\left(\mathrm{i} c_{\mathrm{S}} \omega_{2}|x-z|+1\right)+c_{\mathrm{s}}^{2} \omega_{2}^{2}\right) e^{\mathrm{i}\left(c_{\mathrm{s}} \omega_{1}|x-y|-c_{\mathrm{s}} \omega_{2}|x-z|\right)}\right. \\
& +\left(\mathrm{i} c_{\mathrm{p}} \omega_{1}|x-y|-1\right)\left(\frac{3}{|x-z|^{2}}\left(\mathrm{i} c_{\mathrm{p}} \omega_{2}|x-z|+1\right)+c_{\mathrm{p}}^{2} \omega_{2}^{2}\right) e^{\mathrm{i}\left(c_{\mathrm{p}} \omega_{1}|x-y|-c_{\mathrm{p}} \omega_{2}|x-z|\right)} \\
& -\left(\mathrm{i} c_{\mathrm{s}} \omega_{1}|x-y|-1\right)\left(\frac{3}{|x-z|^{2}}\left(\mathrm{i} c_{\mathrm{p}} \omega_{2}|x-z|+1\right)+c_{\mathrm{p}}^{2} \omega_{2}^{2}\right) e^{\mathrm{i}\left(c_{\mathrm{s}} \omega_{1}|x-y|-c_{\mathrm{p}} \omega_{2}|x-z|\right)} \\
& \left.-\left(\mathrm{i} c_{\mathrm{p}} \omega_{1}|x-y|-1\right)\left(\frac{3}{|x-z|^{2}}\left(\mathrm{i} c_{\mathrm{s}} \omega_{2}|x-z|+1\right)+c_{\mathrm{s}}^{2} \omega_{2}^{2}\right) e^{\mathrm{i}\left(c_{\mathrm{p}} \omega_{1}|x-y|-c_{\mathrm{s}} \omega_{2}|x-z|\right)}\right] \\
& \times \frac{\left(x_{i}-z_{i}\right)^{2}}{|x-y|^{3}|x-z|^{3}} \mathbb{E}\left(f_{i}(y) f_{i}(z)\right) \mathrm{d} y \mathrm{~d} z \\
& \mathbb{E}\left(u_{i 3}\left(x, \omega_{1}\right) \overline{u_{i 1}\left(x, \omega_{2}\right)}\right)=-\frac{1}{16 \pi^{2} \mu \omega_{1}^{2}} \times \\
& \int_{\mathbb{R}^{6}}\left[\left(\frac{3}{|x-y|^{2}}\left(\mathrm{i} c_{\mathrm{s}} \omega_{1}|x-y|-1\right)+c_{\mathrm{S}}^{2} \omega_{1}^{2}\right) e^{\mathrm{i}\left(c_{\mathrm{s}} \omega_{1}|x-y|-c_{\mathrm{s}} \omega_{2}|x-z|\right)}\right. \\
& \left.-\left(\frac{3}{|x-y|^{2}}\left(\mathrm{i} c_{\mathrm{p}} \omega_{1}|x-y|-1\right)+c_{\mathrm{p}}^{2} \omega_{1}^{2}\right) e^{\mathrm{i}\left(c_{\mathrm{p}} \omega_{1}|x-y|-c_{\mathrm{s}} \omega_{2}|x-z|\right)}\right] \\
& \times \frac{\left(x_{i}-y_{i}\right)^{2}}{|x-y|^{3}|x-z|} \mathbb{E}\left(f_{i}(y) f_{i}(z)\right) \mathrm{d} y \mathrm{~d} z
\end{aligned}
$$




$$
\begin{aligned}
& \mathbb{E}\left(u_{i 3}\left(x, \omega_{1}\right) \overline{u_{i 2}\left(x, \omega_{2}\right)}\right)=\frac{1}{16 \pi^{2} \omega_{1}^{2} \omega_{2}^{2}} \times \\
& \int_{\mathbb{R}^{6}}\left[\left(\mathrm{i} c_{\mathrm{s}} \omega_{2}|x-z|+1\right)\left(\frac{3}{|x-y|^{2}}\left(\mathrm{i} c_{\mathrm{S}} \omega_{1}|x-y|-1\right)+c_{\mathrm{S}}^{2} \omega_{1}^{2}\right) e^{\mathrm{i}\left(c_{\mathrm{s}} \omega_{1}|x-y|-c_{\mathrm{s}} \omega_{2}|x-z|\right)}\right. \\
& +\left(\mathrm{i} c_{\mathrm{p}} \omega_{2}|x-z|+1\right)\left(\frac{3}{|x-y|^{2}}\left(\mathrm{i} c_{\mathrm{p}} \omega_{1}|x-y|-1\right)+c_{\mathrm{p}}^{2} \omega_{1}^{2}\right) e^{\mathrm{i}\left(c_{\mathrm{p}} \omega_{1}|x-y|-c_{\mathrm{p}} \omega_{2}|x-z|\right)} \\
& -\left(\mathrm{i} c_{\mathrm{p}} \omega_{2}|x-z|+1\right)\left(\frac{3}{|x-y|^{2}}\left(\mathrm{i} c_{\mathrm{s}} \omega_{1}|x-y|-1\right)+c_{\mathrm{s}}^{2} \omega_{1}^{2}\right) e^{\mathrm{i}\left(c_{\mathrm{s}} \omega_{1}|x-y|-c_{\mathrm{p}} \omega_{2}|x-z|\right)} \\
& \left.-\left(\mathrm{i} c_{\mathrm{s}} \omega_{2}|x-z|+1\right)\left(\frac{3}{|x-y|^{2}}\left(\mathrm{i} c_{\mathrm{p}} \omega_{1}|x-y|-1\right)+c_{\mathrm{p}}^{2} \omega_{1}^{2}\right) e^{\mathrm{i}\left(c_{\mathrm{p}} \omega_{1}|x-y|-c_{\mathrm{s}} \omega_{2}|x-z|\right)}\right] \\
& \times \frac{\left(x_{i}-y_{i}\right)^{2}}{|x-y|^{3}|x-z|^{3}} \mathbb{E}\left(f_{i}(y) f_{i}(z)\right) \mathrm{d} y \mathrm{~d} z \\
& \mathbb{E}\left(u_{i 3}\left(x, \omega_{1}\right) \overline{u_{i 3}\left(x, \omega_{2}\right)}\right)=\frac{1}{16 \pi^{2} \omega_{1}^{2} \omega_{2}^{2}} \int_{\mathbb{R}^{6}}\left[\left(\frac{3}{|x-y|^{2}}\left(\mathrm{i} c_{\mathrm{s}} \omega_{1}|x-y|-1\right)+c_{\mathrm{s}}^{2} \omega_{1}^{2}\right) \times\right. \\
& \left(\frac{3}{|x-z|^{2}}\left(-\mathrm{i} c_{\mathrm{S}} \omega_{2}|x-z|-1\right)+c_{\mathrm{s}}^{2} \omega_{2}^{2}\right) e^{\mathrm{i}\left(c_{\mathrm{s}} \omega_{1}|x-y|-c_{\mathrm{s}} \omega_{2}|x-z|\right)} \\
& +\left(\frac{3}{|x-y|^{2}}\left(\mathrm{i} c_{\mathrm{p}} \omega_{1}|x-y|-1\right)+c_{\mathrm{p}}^{2} \omega_{1}^{2}\right) \times \\
& \left(\frac{3}{|x-z|^{2}}\left(-\mathrm{i} c_{\mathrm{p}} \omega_{2}|x-z|-1\right)+c_{\mathrm{p}}^{2} \omega_{2}^{2}\right) e^{\mathrm{i}\left(c_{\mathrm{p}} \omega_{1}|x-y|-c_{\mathrm{p}} \omega_{2}|x-z|\right)} \\
& -\left(\frac{3}{|x-y|^{2}}\left(\mathrm{i} c_{\mathrm{s}} \omega_{1}|x-y|-1\right)+c_{\mathrm{s}}^{2} \omega_{1}^{2}\right) \times \\
& \left(\frac{3}{|x-z|^{2}}\left(-\mathrm{i} c_{\mathrm{p}} \omega_{2}|x-z|-1\right)+c_{\mathrm{p}}^{2} \omega_{2}^{2}\right) e^{\mathrm{i}\left(c_{\mathrm{s}} \omega_{1}|x-y|-c_{\mathrm{p}} \omega_{2}|x-z|\right)} \\
& -\left(\frac{3}{|x-y|^{2}}\left(\mathrm{i} c_{\mathrm{p}} \omega_{1}|x-y|-1\right)+c_{\mathrm{p}}^{2} \omega_{1}^{2}\right) \times \\
& \left.\left(\frac{3}{|x-z|^{2}}\left(-\mathrm{i} c_{\mathrm{S}} \omega_{2}|x-z|-1\right)+c_{\mathrm{S}}^{2} \omega_{2}^{2}\right) e^{\mathrm{i}\left(c_{\mathrm{p}} \omega_{1}|x-y|-c_{\mathrm{s}} \omega_{2}|x-z|\right)}\right] \\
& \times \frac{\left(x_{i}-y_{i}\right)\left(x_{i}-z_{i}\right)}{|x-y|^{3}|x-z|^{3}} \sum_{j=1}^{3}\left(x_{j}-y_{j}\right)\left(x_{j}-z_{j}\right) \mathbb{E}\left(f_{j}(y) f_{j}(z)\right) \mathrm{d} y \mathrm{~d} z .
\end{aligned}
$$

Observe the above expressions, it is easy to see that $\mathbb{E}\left(\boldsymbol{u}\left(x, \omega_{1}\right) \cdot \overline{\boldsymbol{u}\left(x, \omega_{2}\right)}\right)$ is a linear combination of $I\left(x, \omega_{1}, \omega_{2}\right)$ which is defined by (3.21). A direct application of Lemma 3.5 leads to the following lemma which plays an important role in the proof of the main results.

Lemma 4.7. For $\omega_{1} \geq 1, \omega_{2} \geq 1$, the estimates

$$
\begin{aligned}
& \left|\mathbb{E}\left(\boldsymbol{u}\left(x, \omega_{1}\right) \cdot \overline{\boldsymbol{u}\left(x, \omega_{2}\right)}\right)\right| \leq c_{n}\left(1+\left|\omega_{1}-\omega_{2}\right|\right)^{-n}\left(\omega_{1}+\omega_{2}\right)^{-m} \\
& \left|\mathbb{E}\left(\boldsymbol{u}\left(x, \omega_{1}\right) \cdot \boldsymbol{u}\left(x, \omega_{2}\right)\right)\right| \leq c_{n}\left(\omega_{1}+\omega_{2}\right)^{-n}\left(1+\left|\omega_{1}-\omega_{2}\right|\right)^{-m}
\end{aligned}
$$

holds uniformly for $x \in U$, where $n \in \mathbb{N}$ is arbitrary and $c_{n} \geq 0$ is a constant depending only on $n$. 
Now we are ready to compute the order of $\mathbb{E}\left(|u(x, \omega)|^{2}\right)$. Let $\omega_{1}=\omega_{2}=\omega$ in $\mathbb{E}\left(u_{i j}\left(x, \omega_{1}\right) \overline{u_{i k}\left(x, \omega_{1}\right)}\right)$ for $i, j, k=1,2,3$, a direct application of Lemma 3.7 gives that

$$
\begin{array}{ll}
\mathbb{E}\left(u_{i 1}(x, \omega) \overline{u_{i 2}(x, \omega)}\right)=O\left(\omega^{-(m+1)}\right), & \mathbb{E}\left(u_{i 2}(x, \omega) \overline{u_{i 1}(x, \omega)}\right)=O\left(\omega^{-(m+1)}\right), \\
\mathbb{E}\left(u_{i 2}(x, \omega) \overline{u_{i 2}(x, \omega)}\right)=O\left(\omega^{-(m+1)}\right), & \mathbb{E}\left(u_{i 2}(x, \omega) \overline{u_{i 3}(x, \omega)}\right)=O\left(\omega^{-(m+1)}\right), \\
\mathbb{E}\left(u_{i 3}(x, \omega) \overline{u_{i 2}(x, \omega)}\right)=O\left(\omega^{-(m+1)}\right), &
\end{array}
$$

and

$$
\begin{aligned}
& \mathbb{E}\left(u_{i 1}(x, \omega) \overline{u_{i 1}(x, \omega)}\right)=N_{1 i}^{(3)}(x) \omega^{-m}+O\left(\omega^{-(m+1)}\right), \\
& \mathbb{E}\left(u_{i 1}(x, \omega) \overline{u_{i 3}(x, \omega)}\right)=N_{2 i}^{(3)}(x)(x, \omega) \omega^{-m}+O\left(\omega^{-(m+1)}\right), \\
& \mathbb{E}\left(u_{i 3}(x, \omega) \overline{u_{i 1}(x, \omega)}\right)=N_{3 i}^{(3)}(x, \omega) \omega^{-m}+O\left(\omega^{-(m+1)}\right), \\
& \mathbb{E}\left(u_{i 3}(x, \omega) \overline{u_{i 3}(x, \omega)}\right)=\sum_{j=1}^{3} N_{4 i, j}^{(3)}(x, \omega) \omega^{-m}+O\left(\omega^{-(m+1)}\right),
\end{aligned}
$$

where

$$
\begin{aligned}
N_{1 i}^{(3)}(x) & =b_{1} \int_{\mathbb{R}^{3}} \frac{1}{|x-y|^{2}} \phi(y) \mathrm{d} y, \\
N_{2 i}^{(3)}(x, \omega) & =\int_{\mathbb{R}^{3}}\left(b_{2} e^{\mathrm{i}\left(c_{\mathrm{s}}-c_{\mathrm{p}}\right)|x-y| \omega}-b_{1}\right) \frac{\left(x_{i}-y_{i}\right)^{2}}{|x-y|^{4}} \phi(y) \mathrm{d} y, \\
N_{3 i}^{(3)}(x, \omega) & =\int_{\mathbb{R}^{3}}\left(b_{2} e^{\mathrm{i}\left(c_{\mathrm{p}}-c_{\mathrm{s}}\right)|x-y| \omega}-b_{1}\right) \frac{\left(x_{i}-y_{i}\right)^{2}}{|x-y|^{4}} \phi(y) \mathrm{d} y, \\
N_{4 i, j}^{(3)}(x, \omega) & =\int_{\mathbb{R}^{3}}\left(b_{3}-2 b_{2} \cos \left(\left(c_{\mathrm{s}}-c_{\mathrm{p}}\right)|x-y| \omega\right)\right) \frac{\left(x_{i}-y_{i}\right)^{2}\left(x_{j}-y_{j}\right)^{2}}{|x-y|^{6}} \phi(y) \mathrm{d} y .
\end{aligned}
$$

Here $b_{1}, b_{2}, b_{3}$ are positive constants given by

$$
b_{1}=\frac{1}{128 \pi^{2}} c_{\mathrm{s}}^{4-m}, \quad b_{2}=\frac{\left(c_{\mathrm{s}} c_{\mathrm{p}}\right)^{2}}{128 \pi^{2}}\left(\frac{2}{c_{\mathrm{s}}+c_{\mathrm{p}}}\right)^{m}, \quad b_{3}=\frac{1}{128 \pi^{2}}\left(c_{\mathrm{s}}^{4-m}+c_{\mathrm{p}}^{4-m}\right) .
$$

Therefore

$$
\mathbb{E}\left(|\boldsymbol{u}(x, \omega)|^{2}\right)=T_{\mathrm{E}}^{(3)}(x) \omega^{-m}+O\left(\omega^{-(m+1)}\right),
$$

where

$$
T_{\mathrm{E}}^{(3)}(x)=\left(b_{3}-b_{1}\right) \int_{\mathbb{R}^{3}} \frac{1}{|x-y|^{2}} \phi(y) \mathrm{d} y .
$$

Now we are ready to present the main result of elastic waves for the three-dimensional case.

Theorem 4.8. Let the external source $\boldsymbol{f}$ be a microlocally isotropic Gaussian random vector field which satisfies Assumption $C$. Then for all $x \in U$, it holds almost surely that

$$
\lim _{Q \rightarrow \infty} \frac{1}{Q-1} \int_{1}^{Q} \omega^{m}|\boldsymbol{u}(x, \omega)|^{2} \mathrm{~d} \omega=T_{\mathrm{E}}^{(3)}(x) .
$$

Moreover, the scattering data $T_{\mathrm{E}}^{(3)}(x)$, for $x \in U$, uniquely determine the micro-correlation strength $\phi$ through the linear relation 4.18).

Proof. Using Lemma 3.8 and (4.17), we may follow the same proof as that for the two-dimensional case. The details are omitted here for brevity. 


\section{Conclusion}

We have studied an inverse source scattering problem for the two- and three-dimensional Helmholtz equation and Navier equation. The source is assumed to be a generalized Gaussian random function whose covariance operator is a classical pseudo-differential operator. By an exact expression of the random wave field and microlocal analysis, we derive a linear integral equation which connects the principle symbol of the covariance operator and the amplitude of the scattering data generated from a single realization of the random source. Based on this relationship, we obtain the uniqueness for the recovery of the principle symbol of the random source for the Helmholtz and Navier equations. A possible continuation of this work is to investigate the uniqueness for Maxwell's equations with a distributional source. Since the Green tensor has a higher singularity for the Maxwell equations, a new technique must be developed. Another interesting direction is to study the uniqueness for the inverse random source problems in inhomogeneous media, where the analytical Green function or tensor is not available any more and the present method may not be directly applicable. We hope to be able to report the progress on these problems in the future.

\section{REFERENCES}

[1] S. Acosta, S. Chow , J. Taylor, and V. Villamizar, On the multi-frequency inverse source problem in heterogeneous media, Inverse Problems, 28 (2012), 075013.

[2] S. Arridge, Optical tomography in medical imaging, Inverse Problems, 15 (1999), R41-R93.

[3] R. Albanese and P. Monk, The inverse source problem for Maxwell's equations, Inverse Problems, 22 (2006),10231035.

[4] H. Ammari, G. Bao, and J. Fleming, An inverse source problem for Maxwell's equations in magnetoencephalography, SIAM J. Appl. Math., 62 (2002), 1369-1382.

[5] H. Ammari, E. Bretin, J. Garnier, H. Kang, H. Lee, and A. Wahab, Mathematical Methods in Elasticity Imaging, Princeton University Press: Princeton, 2015.

[6] M. Abramowitz and I. Stegun, Tables of Mathematical Functions, 9th print, New York: Dover, 1970.

[7] A. Badia and T. Nara, An inverse source problem for Helmholtz's equation from the Cauchy data with a single wave number, Inverse Problems, 27 (2011), 105001.

[8] M. Badieirostami, A. Adibi, H.-M. Zhou, and S.-N. Chow, Wiener chaos expansion and simulation of electromagnetic wave propagation excited by a spatially incoherent source, Multiscale Model. Simul., 8 (2010), 591-604.

[9] N. Bleistein and J. K. Cohen, Nonuniqueness in the inverse source problem in acoustics and electromagnetic, J. Math. Phys., 18 (1977), 194-201.

[10] G. Bao, C. Chen, and P. Li, Inverse random source scattering problems in several dimensions, SIAM/ASA J. Uncertainty Quantification, 4 (2016), 1263-1287.

[11] G. Bao, C. Chen, and P. Li, Inverse random source scattering for elastic waves, SIAM J. Numer. Anal., 55 (2017), 2616-2643.

[12] G. Bao, S.-N. Chow, P. Li, and H. Zhou, An inverse random source problem for the Helmholtz equation, Math. Comp., 83 (2014), 215-233.

[13] G. Bao, P. Li, J. Lin, and F. Triki, Inverse scattering problems with multi-frequencies, Inverse Problems, 31 (2015), 093001.

[14] G. Bao, S. Lu, W. Rundell, and B. Xu, A recursive algorithm for multifrequency acoustic inverse source problems, SIAM J. Numer. Anal., 53 (2015), 1608-1628.

[15] G. Bao, J. Lin, and F. Triki, A multi-frequency inverse source problem, J. Differential Equations, 249 (2010), 3443-3465.

[16] G. Bao, P. Li, and Y. Zhao, Stability in the inverse source problem for elastic and electromagnetic waves with multi-frequencies, submitted.

[17] G. Bao and X. Xu, An inverse random source problem in quantifying the elastic modulus of nanomaterials, Inverse Problems, 29 (2013), 015006.

[18] N. Bleistein and J. K. Cohen, Nonuniqueness in the inverse source problem in acoustics and electromagnetics, J. Math. Phys., 18 (1977), 194-201.

[19] P. Caro, T. Helin, and M. Lassas, Inverse scattering for a random potential, arXiv:1605.08710, 2016.

[20] J. Cheng, V. Isakov, and S. Lu, Increasing stability in the inverse source problem with many frequencies, J. Differential Equations, 260 (2016), 4786-4804.

[21] P. G. Ciarlet, Mathematical Elasticity, vol. I : Three-Dimensional Elasticity, Studies in Mathematics and its Applications, North-Holland, Amsterdam, 1988.

[22] D. Colton and R. Kress, Inverse Acoustic and Electromagnetic Scattering Theory, 3rd ed., Springer, Berlin, 2013. 
[23] H. Cramer and M. Leadbetter, Stationary and Related Stochastic Processes, New York: John Wiley and Sons, 1967.

[24] A. Devaney, The inverse problem for random sources, J. Math. Phys., 20 (1979), 1687-1691.

[25] A. Devaney, E. Marengo, and M. Li, Inverse source problem in nonhomogeneous background media, SIAM J. Appl. Math., 67 (2007), 1353-1378.

[26] A. Devaney and G. Sherman, Nonuniqueness in inverse source and scattering problems, IEEE Trans. Antennas Propag., 30 (1982), 1034-1037.

[27] M. Eller and N. Valdivia, Acoustic source identification using multiple frequency information, Inverse Problems, 25 (2009), 115005.

[28] A. Fokas, Y. Kurylev, and V. Marinakis, The unique determination of neuronal currents in the brain via magnetoencephalogrphy, Inverse Problems, 20 (2004), 1067-1082.

[29] L. Höremainder, The Analysis of Linear Partial Differential Operators, vol. III, Berlin: Springer-Verlag, 1985.

[30] K.-H. Hauer, L. Kühn, and R. Potthast, On uniqueness and non-uniqueness for current reconstruction from magnetic fields, Inverse Problems, 21 (2005), 955-967.

[31] T. Helin, M. Lassas, and L. Päivärinta, Inverse acoustic scattering problem in half-space with anisotropic random impedance, J. Differential Equations, 262 (2017), 3139-3168.

[32] V. Isakov, Inverse Source Problems, AMS, Providence, RI, 1989

[33] D. Jerison and C. E. Kenig, The inhomogeneous Dirichlet problem in Lipschitz domains, J. Funct. Anal., 130 (1995), 161-219.

[34] J. Kaipio and E. Somersalo, Statistical and Computational Inverse Problems, Springer-Varlag, New York, 2005.

[35] L. D. Landau and E. M. Lifshitz, Theory of Elasticity, Pergamon Press, New York, 1959.

[36] P. Li, An inverse random source scattering problem in inhomogeneous media, Inverse Problems, 27 (2011), 035004.

[37] M. Li, C. Chen and P. Li, Inverse random source scattering for the Helmholtz equation in inhomogeneous media, Inverse Problems, 34 (2018), 015003.

[38] P. Li and G. Yuan, Increasing stability for the inverse source scattering problem with multi-frequencies, Inverse Problems and Imaging, 11 (2017), 745-759.

[39] N. N. Lebedev, Special Functions and Their Applications, Dover Publications, INC. New York, 1972.

[40] M. Lassas, L. Päivärinta, and E. Saksman, Inverse scattering problem for a two dimensional random potential, Commun. Math. Phys., 279 (2008), 669-703.

[41] E. Marengo and A. Devaney, The inverse source problem of electromagnetics: Linear inversion formulation and minimum energy solution, IEEE Trans. Antennas and Propagation, 47 (1999), 410-412.

[42] J. Michaels and Y.-H. Pao, The inverse source problem for an oblique force on an elastic plate, J. Acoust. Soc. Am., 77 (1985), 2005-2011.

[43] T. Nara, J. Oohama, M. Hashimoto, T. Takeda, and S. Ando, Direct reconstruction algorithm of current dipoles for vector magnetoencephalography and electroencephalography, Phys. Med. Biol. 52 (2007), 3859-3879.

[44] J. Tittelfitz, An inverse source problem for the elastic wave in the lower-half space, SIAM J. Appl. Math., 75 (2015), 1599-1619.

[45] D. Zhang and Y. Guo, Fourier method for solving the multi-frequency inverse acoustic source problem for the Helmholtz equation, Inverse Problems, 31 (2015), 035007.

School of Mathematics and Statistics, Changsha University of Science and Technology, Changsha, 410114, P.R. China.

E-mail address: lij1@amss.ac.cn

Department of Mathematics and Statistics, University of Helsinki, Helsinki, Finland.

E-mail address: tapio.helin@helsinki.fi

Department of Mathematics, Purdue University, West Lafayette, Indiana 47907, USA.

E-mail address: lipeijun@math.purdue.edu 